Prepared in cooperation with the U.S. Agency for International Development

Grassland Live Fractional Cover Map Creation and Geographic Information System (GIS) Analysis for Rangeland Management Supporting Kenya Northern Rangelands Trust Conservancies

Open-File Report 2021-1001 
Cover. Typical Meibae Conservancy landscape within the Northern Rangelands Trust (NRT) Conservancies, Kenya. November 30, 2019. Photograph by Elijah Ramsey, III, Oceanographer; U.S Geological Survey. 


\section{Grassland Live Fractional Cover Map Creation and Geographic Information System (GIS) Analysis for Rangeland Management Supporting Kenya Northern Rangelands Trust Conservancies}

Amina Rangoonwala and Elijah Ramsey, III

Prepared in cooperation with the

U.S. Agency for International Development

Open-File 2021-1001 


\section{U.S. Geological Survey, Reston, Virginia: 2021}

For more information on the USGS - the Federal source for science about the Earth, its natural and living resources, natural hazards, and the environment-visit https://www.usgs.gov or call 1-888-ASK-USGS.

For an overview of USGS information products, including maps, imagery, and publications, visit https://store.usgs.gov.

Any use of trade, firm, or product names is for descriptive purposes only and does not imply endorsement by the U.S. Government.

Although this information product, for the most part, is in the public domain, it also may contain copyrighted materials as noted in the text. Permission to reproduce copyrighted items must be secured from the copyright owner.

Suggested citation:

Rangoonwala, A., and Ramsey, E., III, 2021, Grassland live fractional cover map creation and Geographic Information System (GIS) analysis for rangeland management supporting Kenya Northern Rangelands Trust Conservancies: U.S. Geological Survey Report 2021-1001, 59 p., https://doi.org/10.3133/ofr20211001.

ISSN 2331-1258 (online) 


\section{Contents}

Abstract

Section I-Image Processing Training Workshop ...............................................................................

1. Downloading Sentinel-2 image data using Copernicus Open Access Hub ........................3

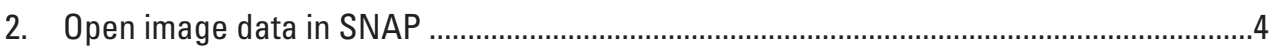

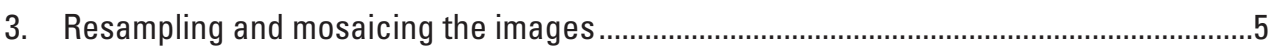

3A. Resample images to be used in the mosaic ........................................................

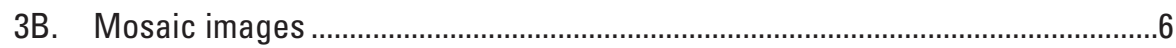

4. Creating modified soil-adjusted vegetation index 2 (MSAVI2) .........................................

4A. Multiply B4 and B8 by 100.0 to scale output......................................................

4B. Calculate MSAVI2 with Band Maths ................................................................

4C. Optical Image Calibration ...................................................................................

5. Creation of Live Vegetation Fractional Cover Map ............................................................

5A. Using the developed model in the OFR as stated in 4C ....................................

5B. Convert the VegMap from decimal format to integer (8U) format .......................

6. Create the 2019-2018 Difference VegMap map .........................................................10

6A. To copy an image in another SNAP product image using Band Maths.............10

6B. Calculate the 2019Veg-2018Veg Difference.....................................................10

6C. Transforming the decimal VegDiff image to an integer format...........................11

6D. Limit the upper VegDiff to 100 percent ..............................................................11

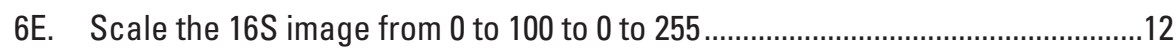

$6 \mathrm{~F}$. Convert the $16 \mathrm{~S}$ band to $8 \mathrm{U}$ numerical format...................................................12

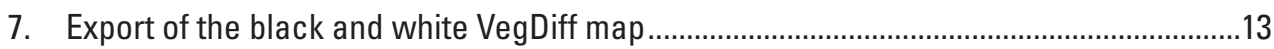

8. Create a color ramp in SNAP for the LFC status and change map ...................................14

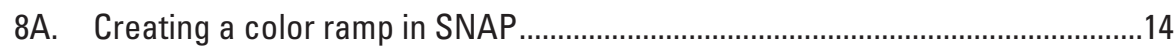

8B. Convert the $0-100$ percent breakpoints to $0-255$ percent breakpoints (two-tile breakpoints) ............................................................................................15

9. Color renditions across raster files with different data types ........................................16

9A. Application of real data format (32-bit or 16-bit) breakpoints to $8 \mathrm{U}$ image

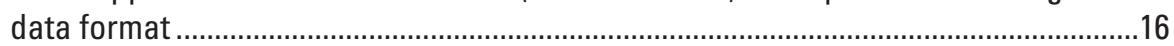

9B. Application of the $8 \mathrm{U}$ breakpoints to the $8 \mathrm{U}$ image data format.........................16

10. Atmospheric correction of Sentinel-2 images using ESA software .............................18

10A. Install Sen2Cor (SNAP atmospheric correction software).............................18

10B. The atmospheric correction process ………………....................................18

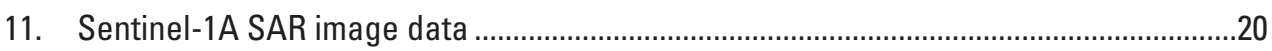

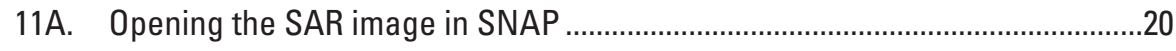

11B. Calibrate the SAR image data to sigma naught ...............................................20

11C. Perform terrain correction and spatial resampling of the calibrated

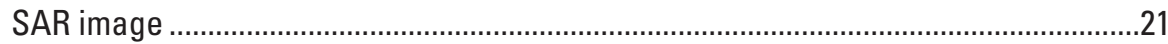

11D. Perform Speckle Filtering of the Calibrate and Terrain Corrected

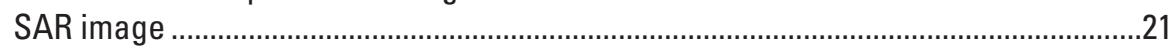

12. Create mosaic of the four radar scenes processed with speckle filter........................22

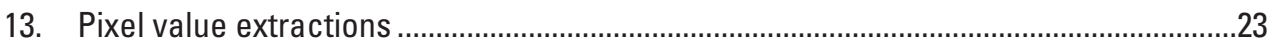

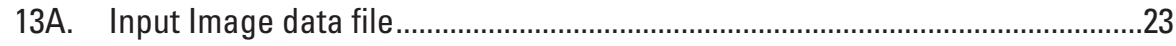

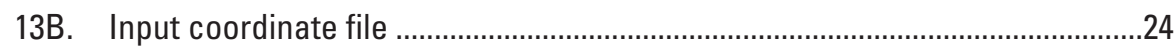




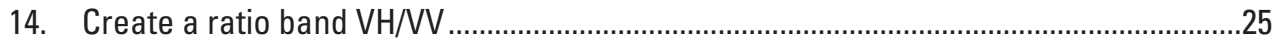

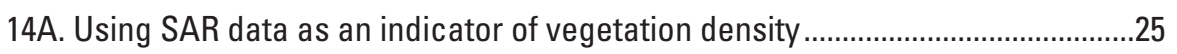

15. Create a subset to match the subset of the 2018 S1A mosaic .........................................26

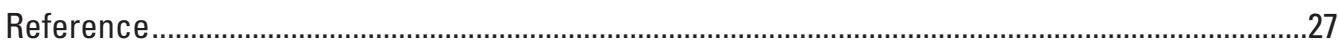

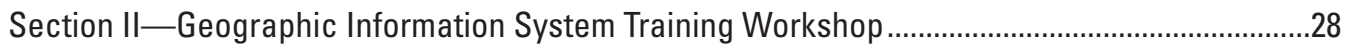

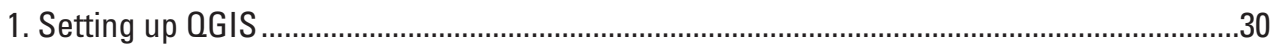

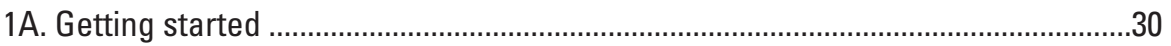

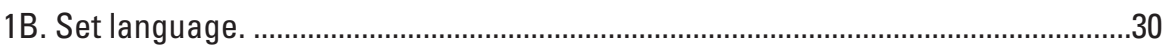

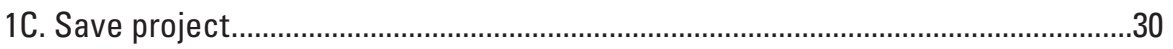

1D. Set Coordinate Reference System ..........................................................................

2. Add Google Maps layers (Google Earth) in QGIS .........................................................32

2A. The Open Street Map is provided as a default XYZ tile or is available in the

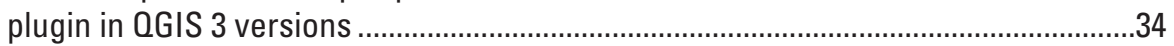

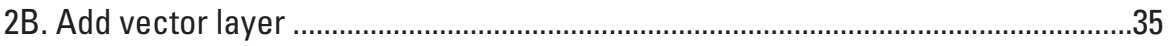

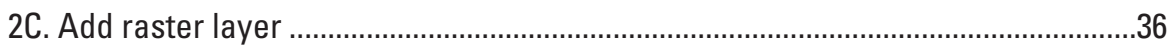

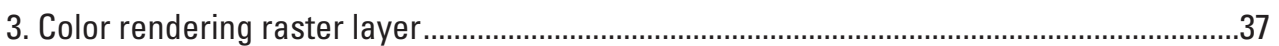

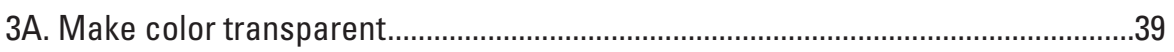

3B. Color rendering raster layer with gradient stops ...................................................40

3C. Save the created color ramp and apply it to the black and white image..............41

3D. The color ramp can also be saved as a *.txt and a *.qml ...................................42

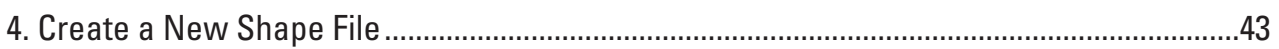

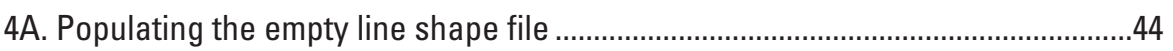

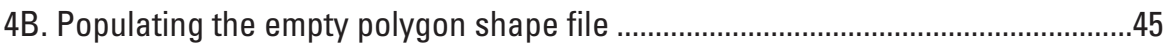

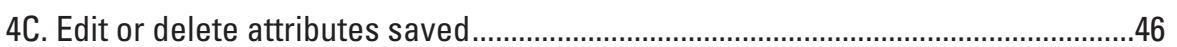

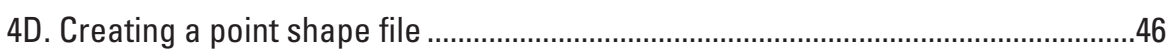

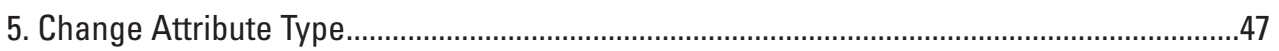

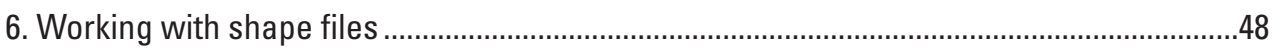

6A. How to formulate query expressions ………………………………………….....

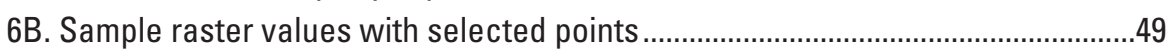

7. Clip raster, raster calculation classification.....................................................................5

7A. Clip raster covering the area of interest for queries ..............................................50

7B. Convert map range ...................................................................................................51

7C. Raster calculator, selecting pixels with LFC coverage with user defined

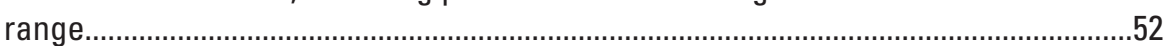

8. Creating buffer zones.........................................................................................................

8A. Buffer zone of Kenya protected areas...............................................................53

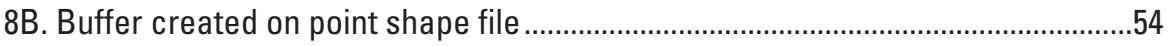

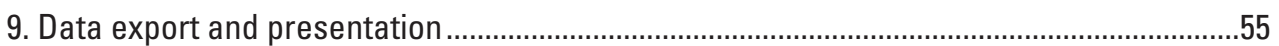

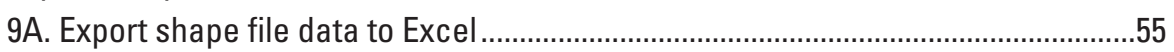

9B. Data export to view in Google Earth Pro .............................................................56

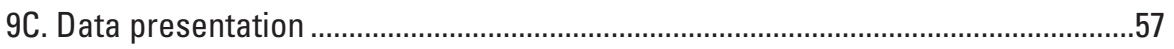

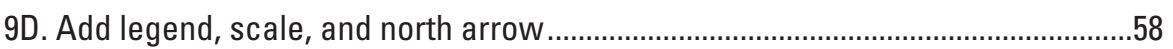

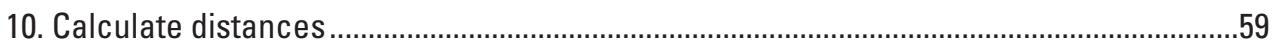




\section{Videos}

[Available at https://dx.doi.org/10.3133/ofr20211001]

1. SeNtinel's Application Platform (SNAP)

2. Quantum Geographic Information System (QGIS) 



\title{
Grassland Live Fractional Cover Map Creation and Geographic Information System (GIS) Analysis for Rangeland Management Supporting Kenya Northern Rangelands Trust Conservancies
}

\author{
Amina Rangoonwala' and Elijah Ramsey, III
}

\begin{abstract}
The handbooks and synchronized MP4 recordings provide hands-on instruction for creating and analyzing vegetation live fractional cover (LFC) maps. The methods and protocols used in the instruction materials follow those developed and recorded in Rangoonwala and Ramsey (2019). The LFC mapping and geographic information system (GIS) analyses highlight the consortium of rangeland conservancies covering the semiarid central region of Kenya (approximately 44,000 square kilometers).

The instruction materials are separated into two parts: processing and map-product creation based on remote-sensing images and GIS analyses of the created maps for rangeland management. The image processing is conducted using the advanced and professional software package SeNtinels Application Platform (SNAP) that is supported and maintained by the European Space Agency. SNAP is a free image analyses software package available for download. It is largely icon driven but offers simple to advanced program inserts and batch processing. The GIS analyses are conducted using the software package Quantum Geographic Information System (QGIS), another free and
\end{abstract}

downloadable software. QGIS is compatible with numerous software, including the Esri suite of ArcGIS software and database structure. The image data includes both high-spatial-resolution Sentinel-2 optical data and Sentinel-1 synthetic aperture radar (SAR). Both datasets are freely available via a public portal that is maintained by the European Space Agency.

The image-processing instruction handbook covers all aspects of acquiring and processing satellite-image data and importing vector-data sources into SNAP. The GIS analysis handbook covers final creation of map products from the maps created in SNAP and creation of GIS procedures in QGIS that are needed to manage the rangeland resources for wildlife and pastoral grazing. Although focused on the Kenyan conservancies and their semiarid environment, the processing methods and procedures are applicable for similar environments and management, and to a large part, even for integrated mapping and GIS functionality of any managed landscape resource.

The instruction handbooks are synchronized to MP4 training videos created with U.S. Geological Survey-licensed Camtasia 9 software.

The workbook and MP4 video combinations are suitable for a single user or a workshop setting.

\footnotetext{
${ }^{1}$ Cherokee Nation Technologies Contractor to the U.S. Geological Survey

${ }^{2}$ U.S. Geological Survey
} 



\section{Section I-Image Processing Training Workshop}

\section{Mapping Live Grassland Cover with SeNtinel's Application Platform (SNAP) Processing of Satellite Optical and Synthetic Aperture Radar (SAR) Image Data}

This Remote Sensing workshop is designed for spatial analysis personnel for creating live vegetation fractional cover maps with SeNtinel's Application Platform (SNAP) software. The workshop provides the skills needed to:

- Search and import Optical and Synthetic Aperture Radar (SAR) image data.

- Use the image processing toolset to transform the satellite optical image data to maps that represent live vegetation cover.

- Use the specialized SAR image processing toolset to create maps from satellite SAR data indicating the vegetation cover density.

- Add value to the produced maps by portraying thematic information with color rendering.

Objective:

- Although the procedures learned can be applied to remote sensing processing of any raster image, this course is specific to the production of live cover status and change maps in the management of the Northern Rangelands Trust (NRT) natural resources. 
2 Grassland Live Fractional Cover Map Creation and Geographic Information System (GIS) Analysis

\section{Optical image data processing- Sentinel 2}




\section{Downloading Sentinel-2 image data using Copernicus Open Access Hub.}

Note that the image data can be downloaded from the ESA "Copernicus Open Access Hub" NOT the "Copernicus data service Hub" site. Registration is required to download data. https://scihub.copernicus.eu/dhus/\#/home

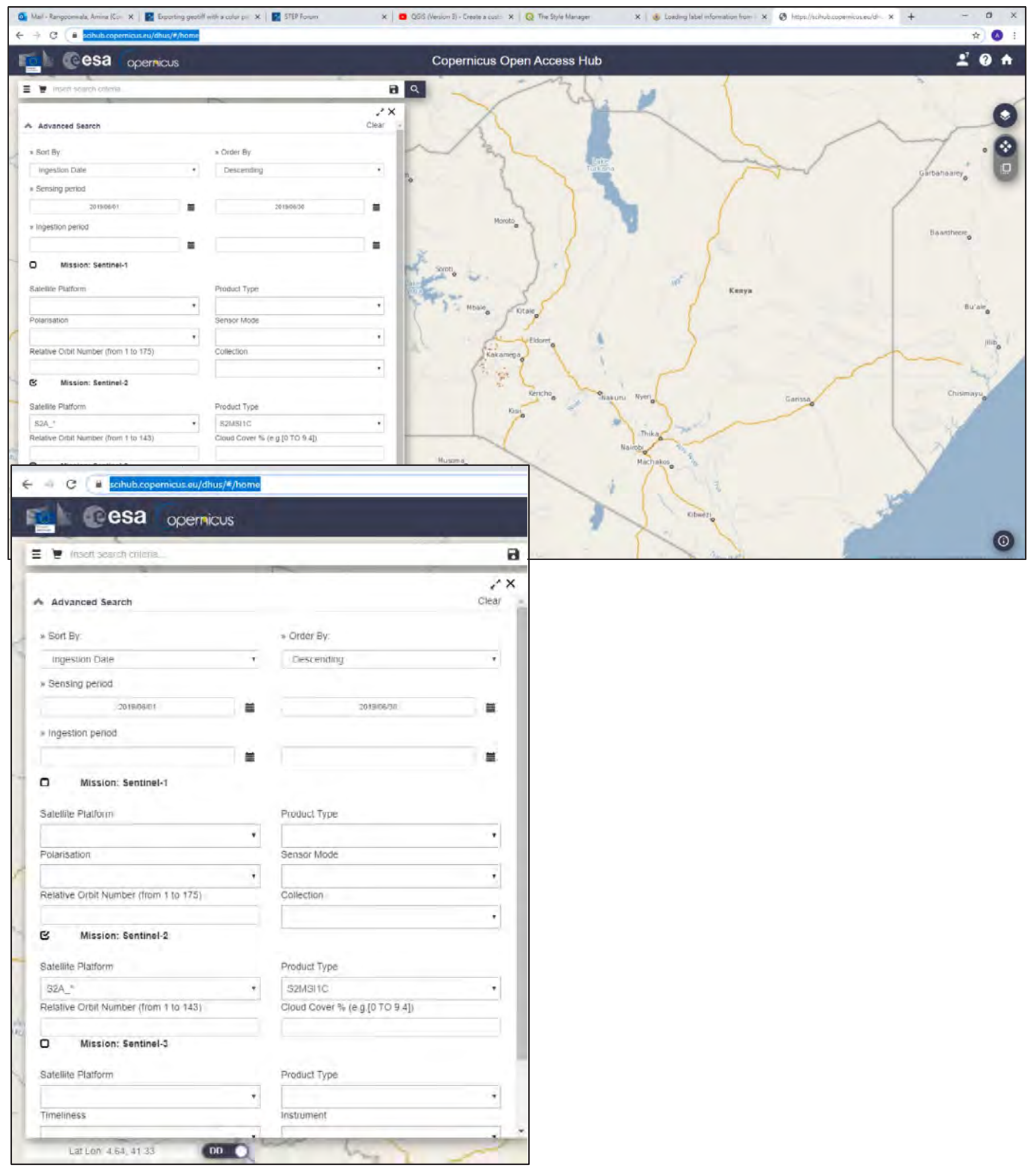




\section{Open image data in SNAP.}

Open SNAP software. Go to Menu>File>Import $>$ Optical Sensors $>$ Sentinel 2. Select S2-MSI-L2A; the dialog window shown below and go to the folder where the data resides; select the *.xml file (MTD_MSIL2A).

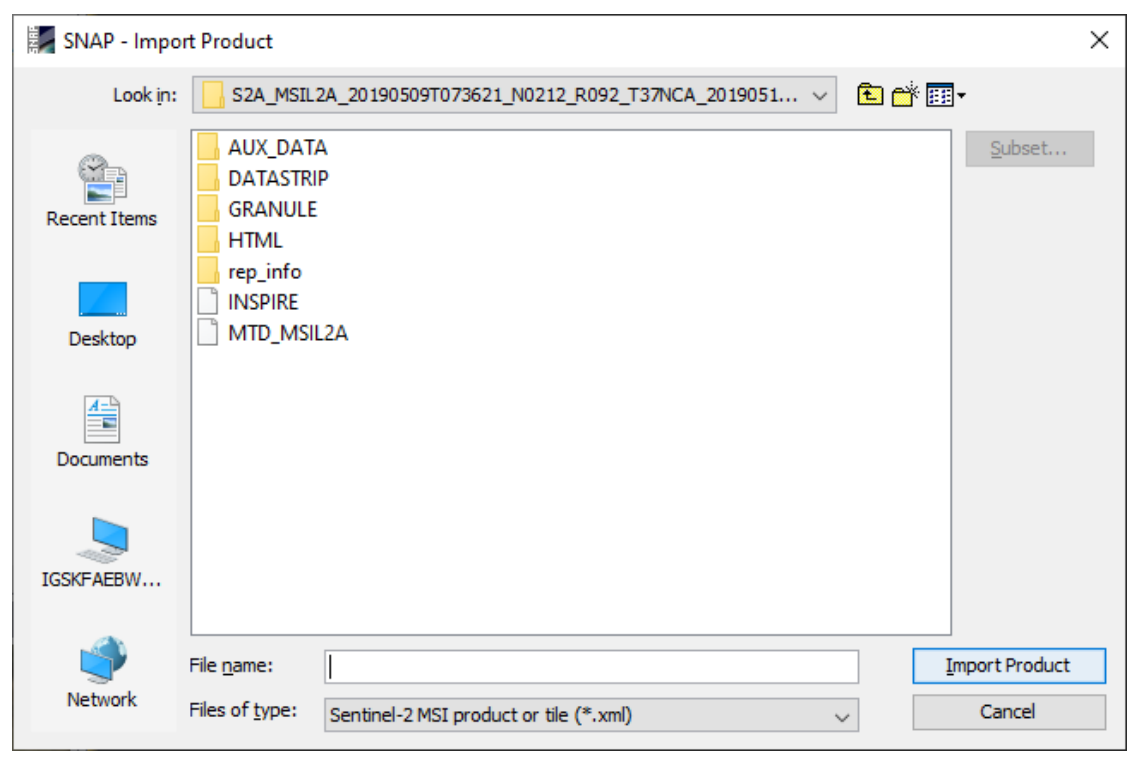

Once the image is loaded in SNAP, right click on the product file and select open RGB window; select false color infrared from the drop down in the Select RGB channels dialog box.

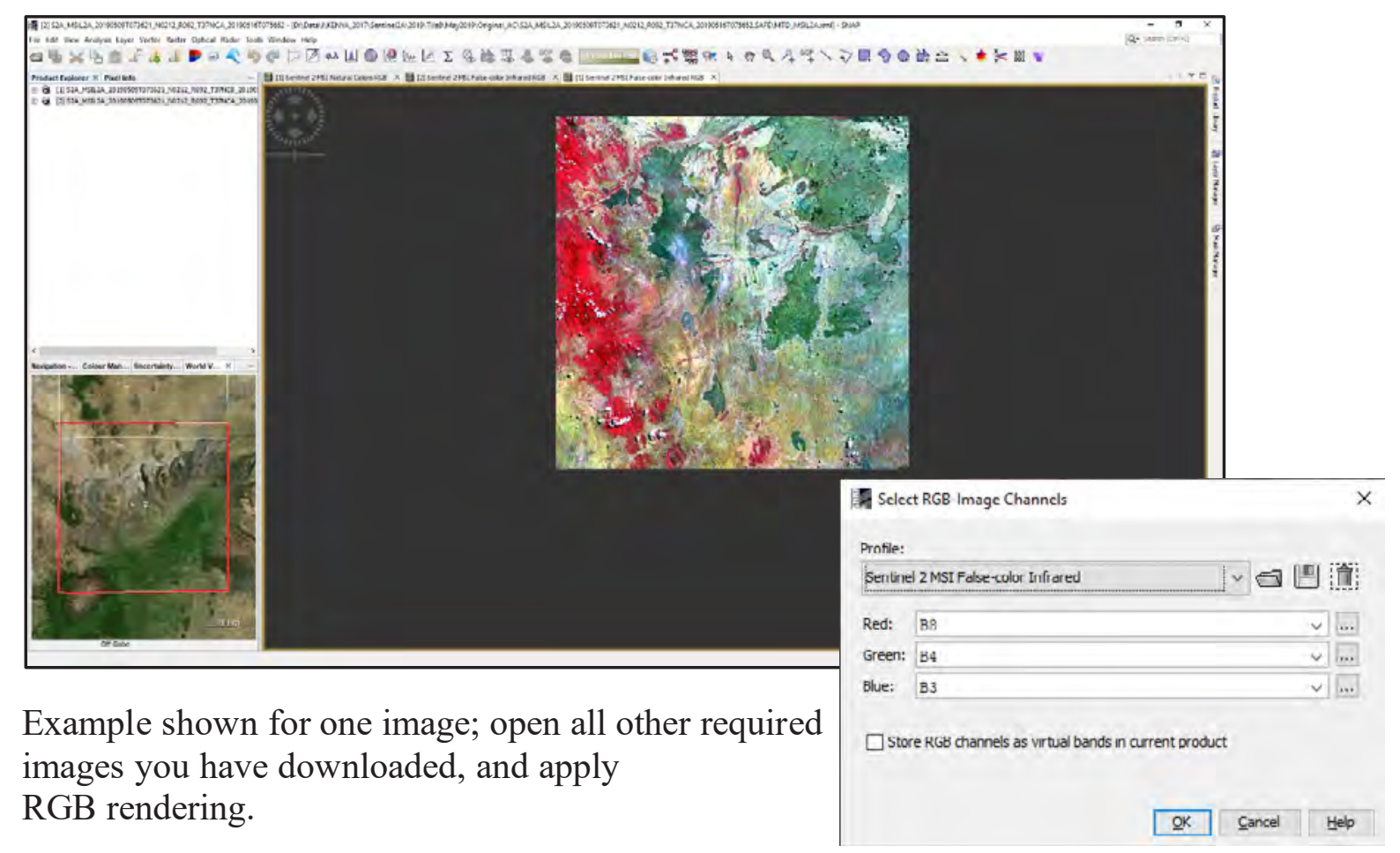




\section{Resampling and mosaicing the images.}

3A. Resample images to be used in the mosaic.

Menu $>$ Raster $>$ Geometric Operations $>$ Resampling.
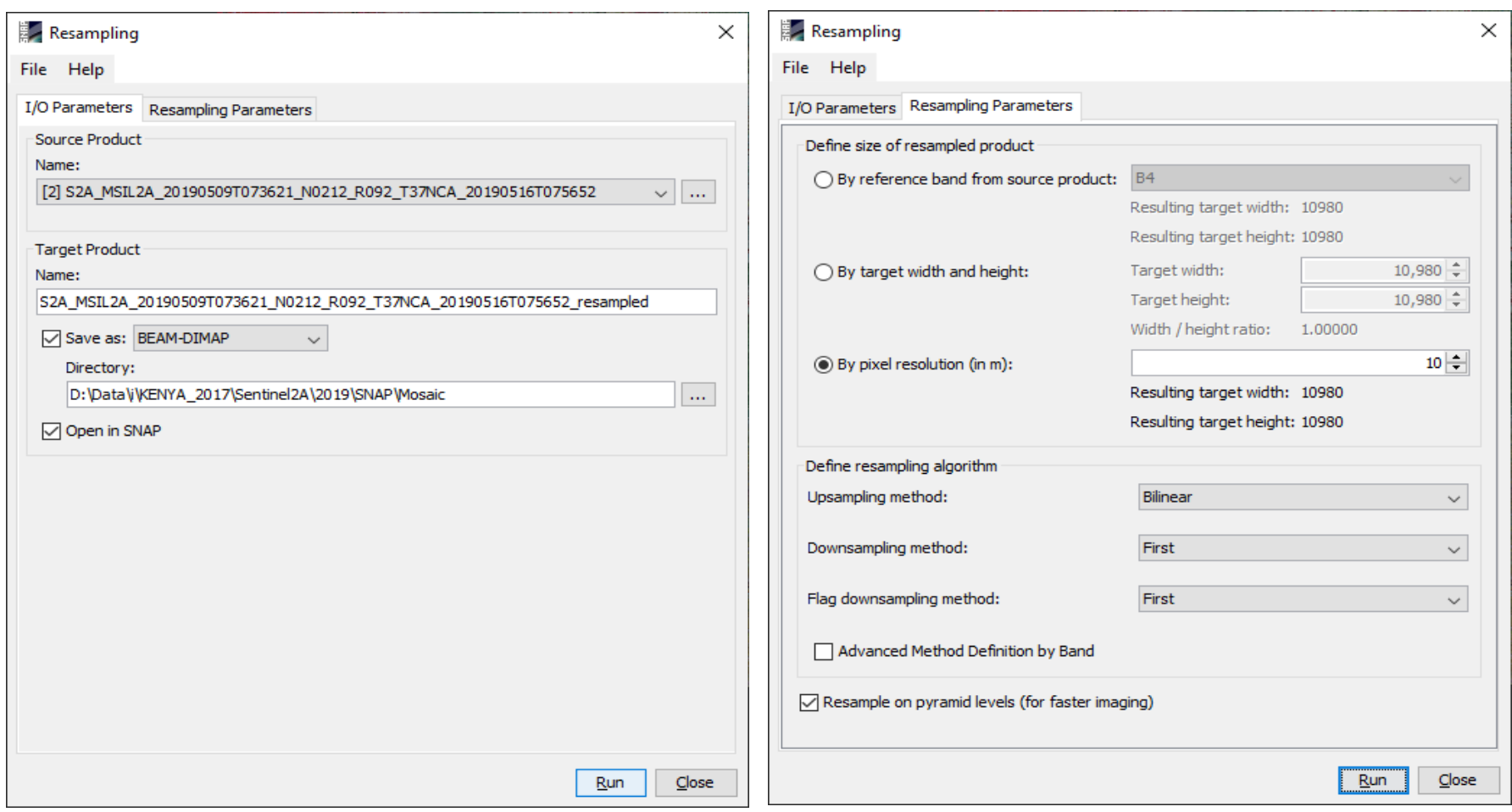

Apply this resampling process to all images. 


\section{B. Mosaic images.}

Go to Menu $>$ Raster $>$ Geometric Operations $>$ Mosaicing.

Select the two resampled images for mosaicing. Follow the steps shown below.

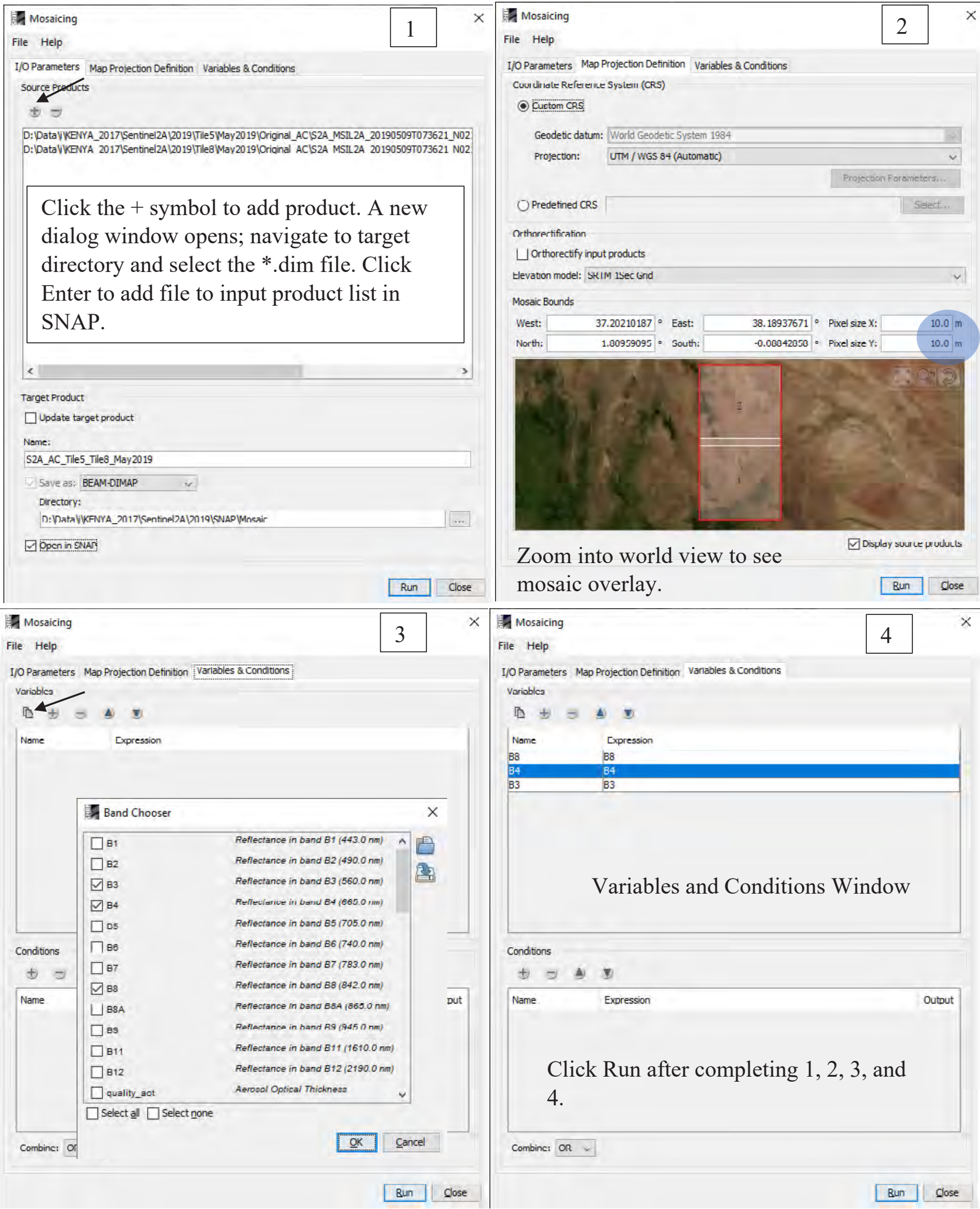




\section{Creating modified soil-adjusted vegetation index 2 (MSAVI2).}

\section{A. Multiply B4 and B8 by $\mathbf{1 0 0 . 0}$ to scale output.}

Right click on the mosaic product to get Band Maths dialog box.

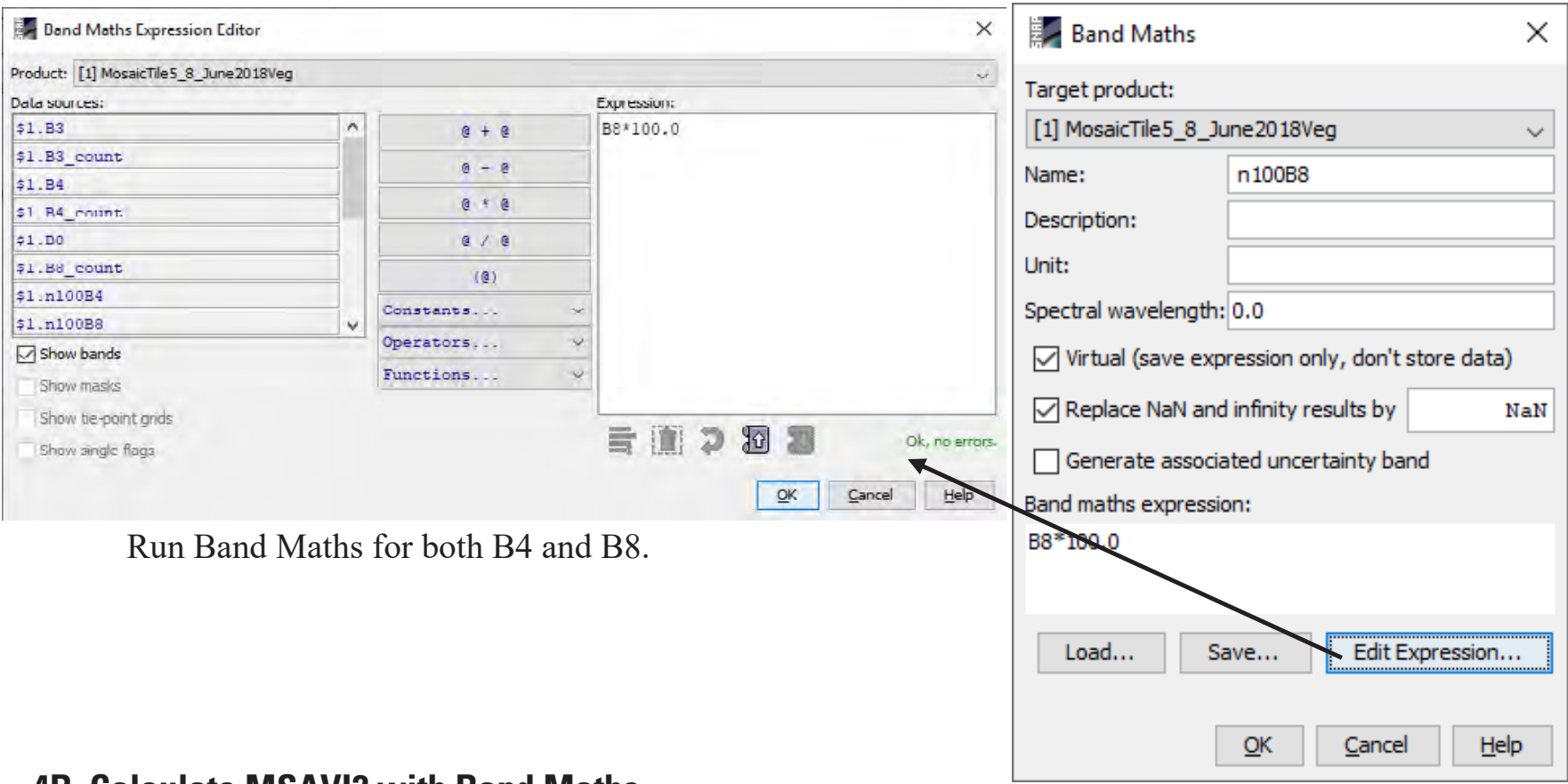

4B. Calculate MSAVI2 with Band Maths.

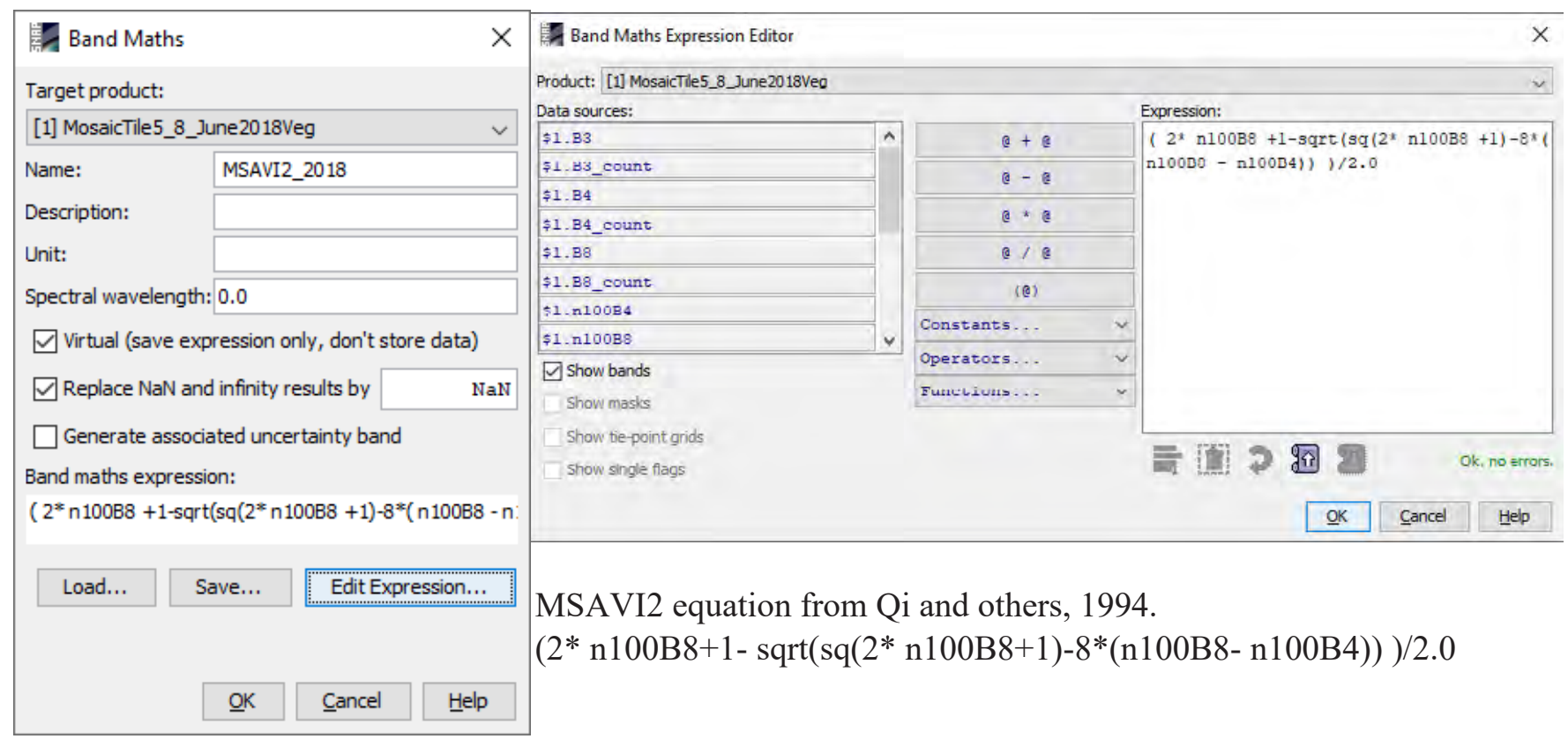


4B. Continued. The MSAVI2 processor in SNAP gives the same result as in Rangoonwala and Ramsey (2019), who used the equation based on Qi and others (1994).

Menu $>$ Optical $>$ thematic land processing $>$ vegetation radiometric indices.

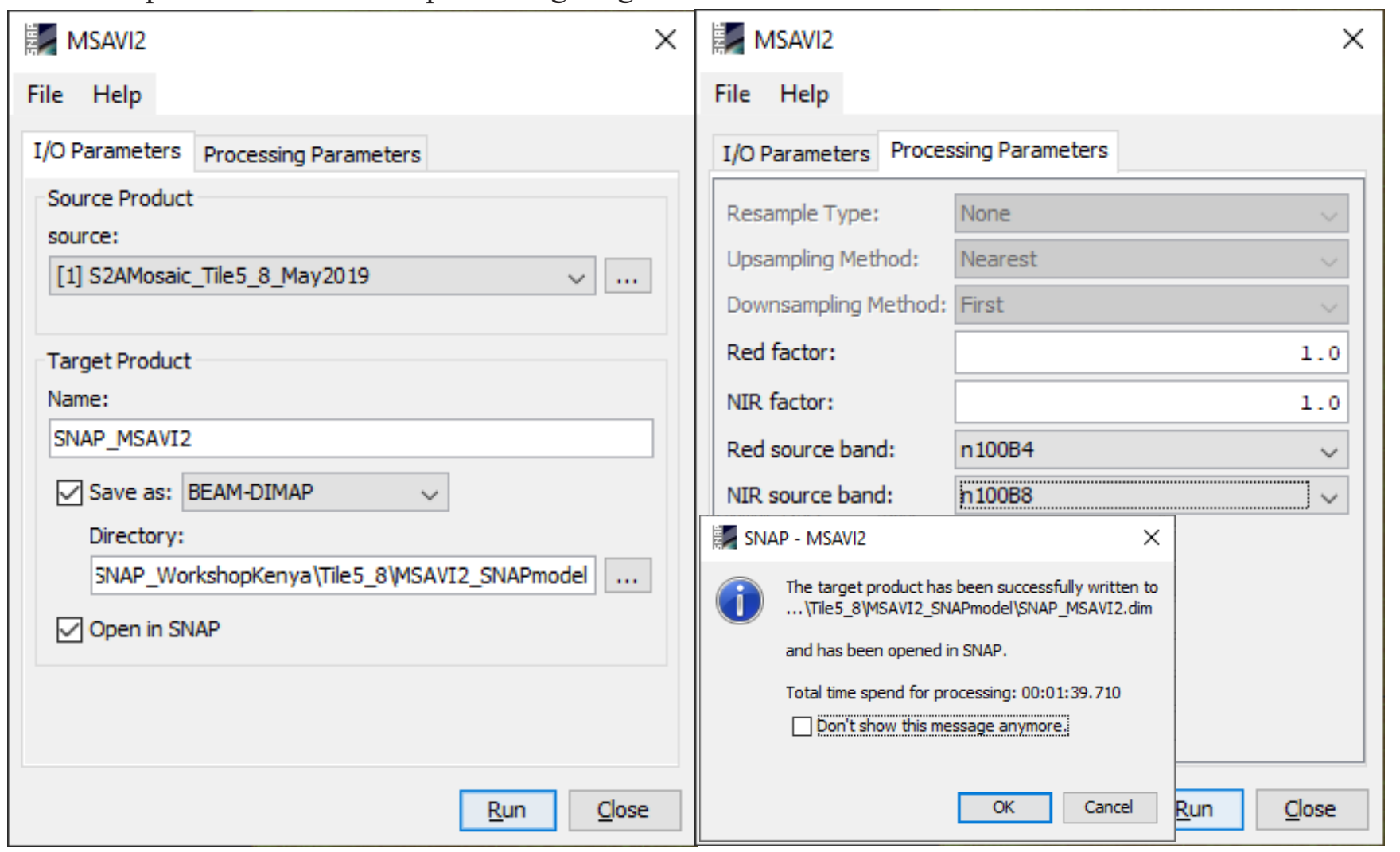

\section{C. Optical Image Calibration.}

Using the developed model discussed in Rangoonwala and Ramsey (2019) (page 16, equation 4), to convert satellite MSAVI2 to Field MSAVI2 (MSAVI2_2018+0.0018)/0.877).

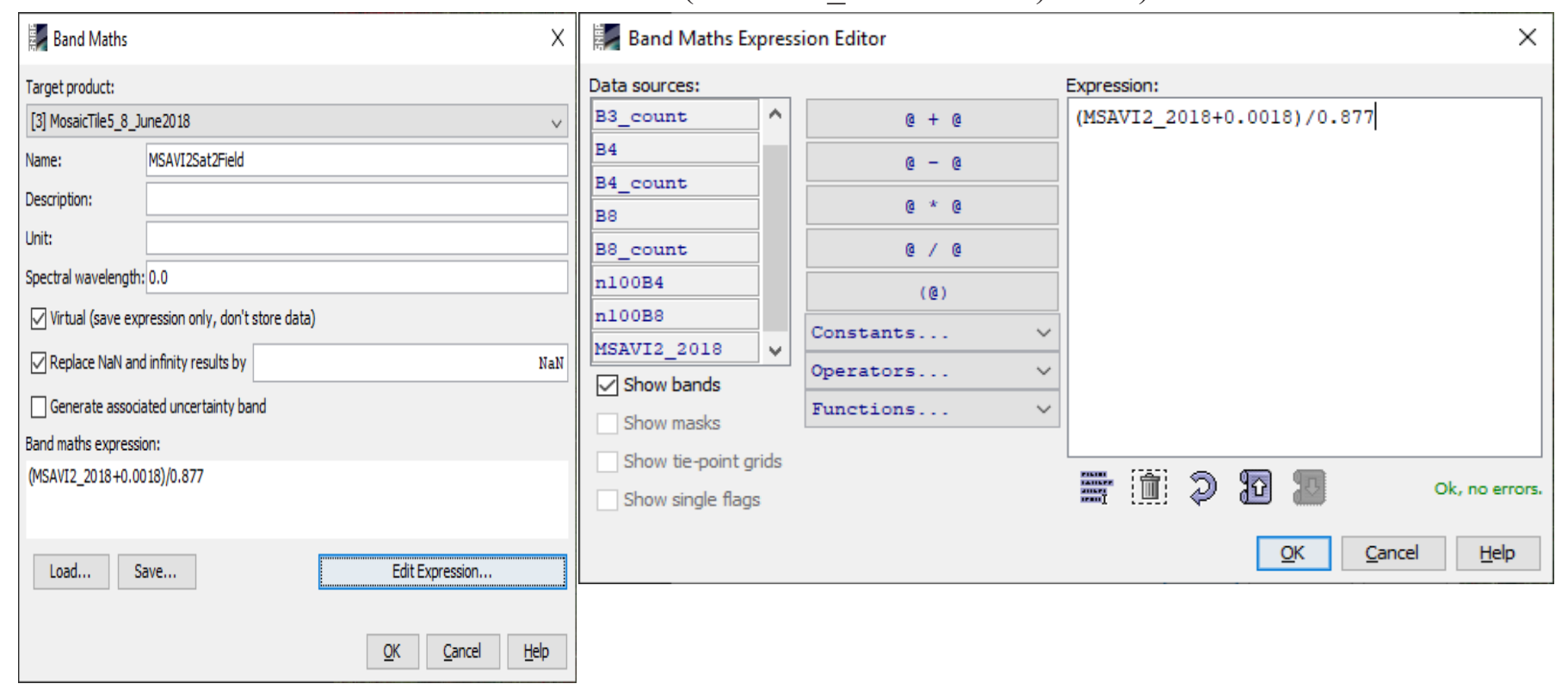




\section{Creation of Live Vegetation Fractional Cover Map.}

\section{A. Using the developed model in the OFR as stated in 4C.}

Use equation 3 on page 16 of Rangoonwala and Ramsey (2019) to convert Field MSAVI2 to Field Web Cam LFC (VegMap) (103.89*MSAVI2Sat2Field), while multiplying the VegMap by 2.55 to stretch the $0-100$ percent range to the full $8 \mathrm{U}$ range of $0-255$.

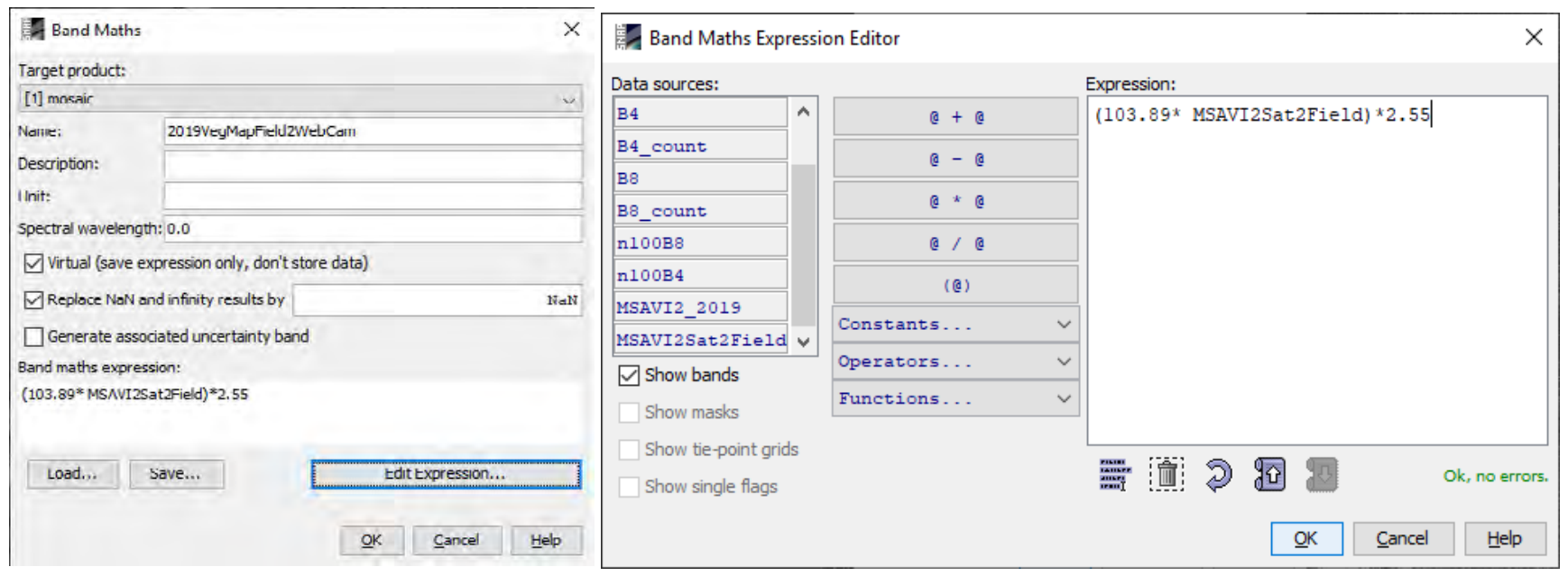

5B. Convert the VegMap from decimal format to integer (8U) format.

Menu $>$ Raster $>$ Data Conversion $>$ Convert Datatype.

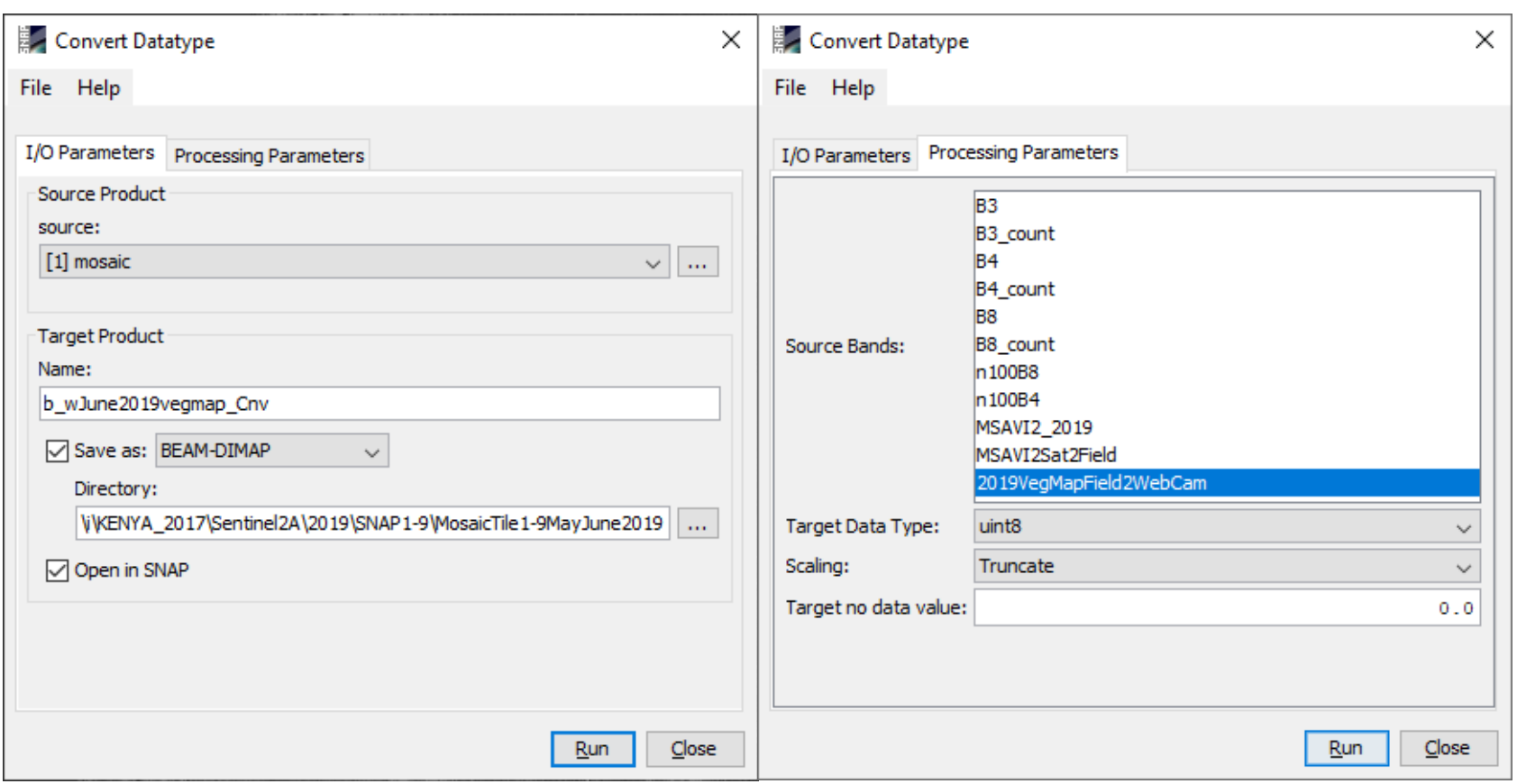

Note: Use the same procedure to calculate the 2019 VegMap (LFC).

After the convert process finishes, open the ${ }^{*}$ C Cnv.data folder. The name of the *.img will be the name of the band converted with the *.img extension. This Veg map *.img file will be used in QGIS. 


\section{Create the 2019-2018 Difference VegMap map.}

\section{A. To copy an image in another SNAP product image using Band Maths.}

To create a difference image, it is important that both the 2018 and 2019 vegetation maps be in the same SNAP product (SNAP database). Follow the screen captures below.

\begin{tabular}{l}
\hline Band Maths \\
Target product: \\
[1] MosaicTile5_8_June2018Veg \\
Name: \\
Description: \\
Unit: \\
Spectral wavelength: 0.0 \\
$\square$ Virtual (save expression only, don't store data) \\
$\square$ Replace NaN and infinity results by \\
$\square$ Generate associated uncertainty band \\
Band maths expression: \\
\\
Load... \\
Save... \\
\end{tabular}

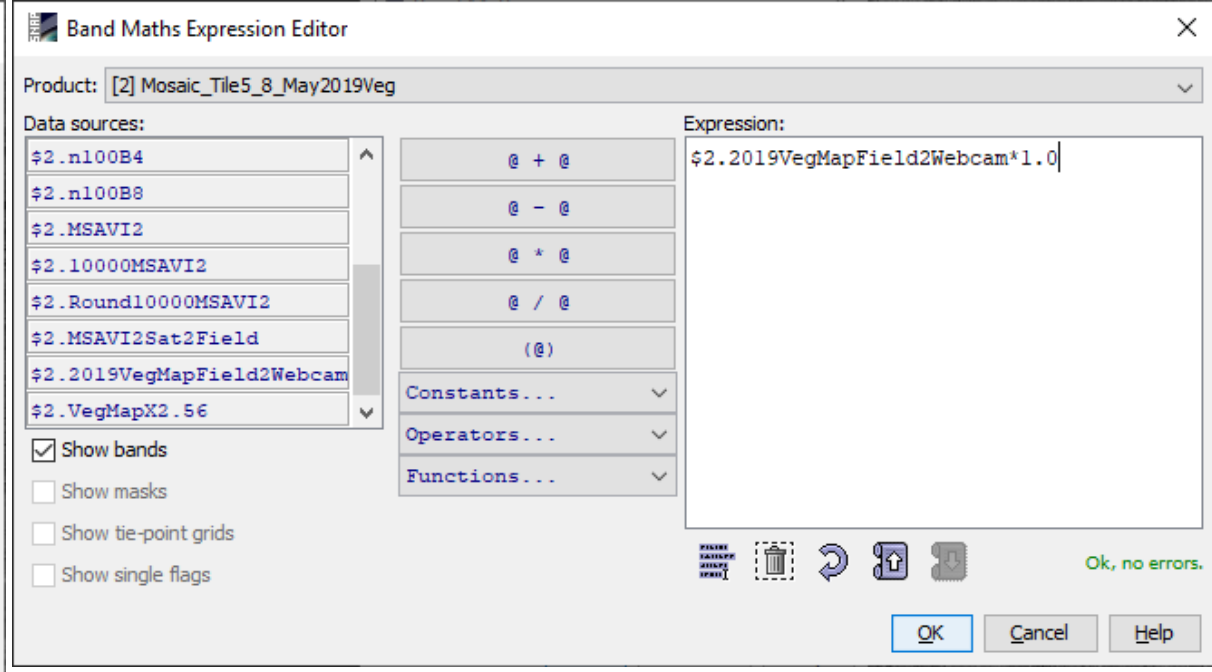

Copy Mosaic_Tile5_8_May2019Veg to Mosaic_Tile5_8_June2018Veg Important: Save the session. Otherwise, problems may occur when opening files. Message appears in SNAP when copying 2019VegMap using band math.

\section{B. Calculate the 2019Veg-2018Veg Difference.}

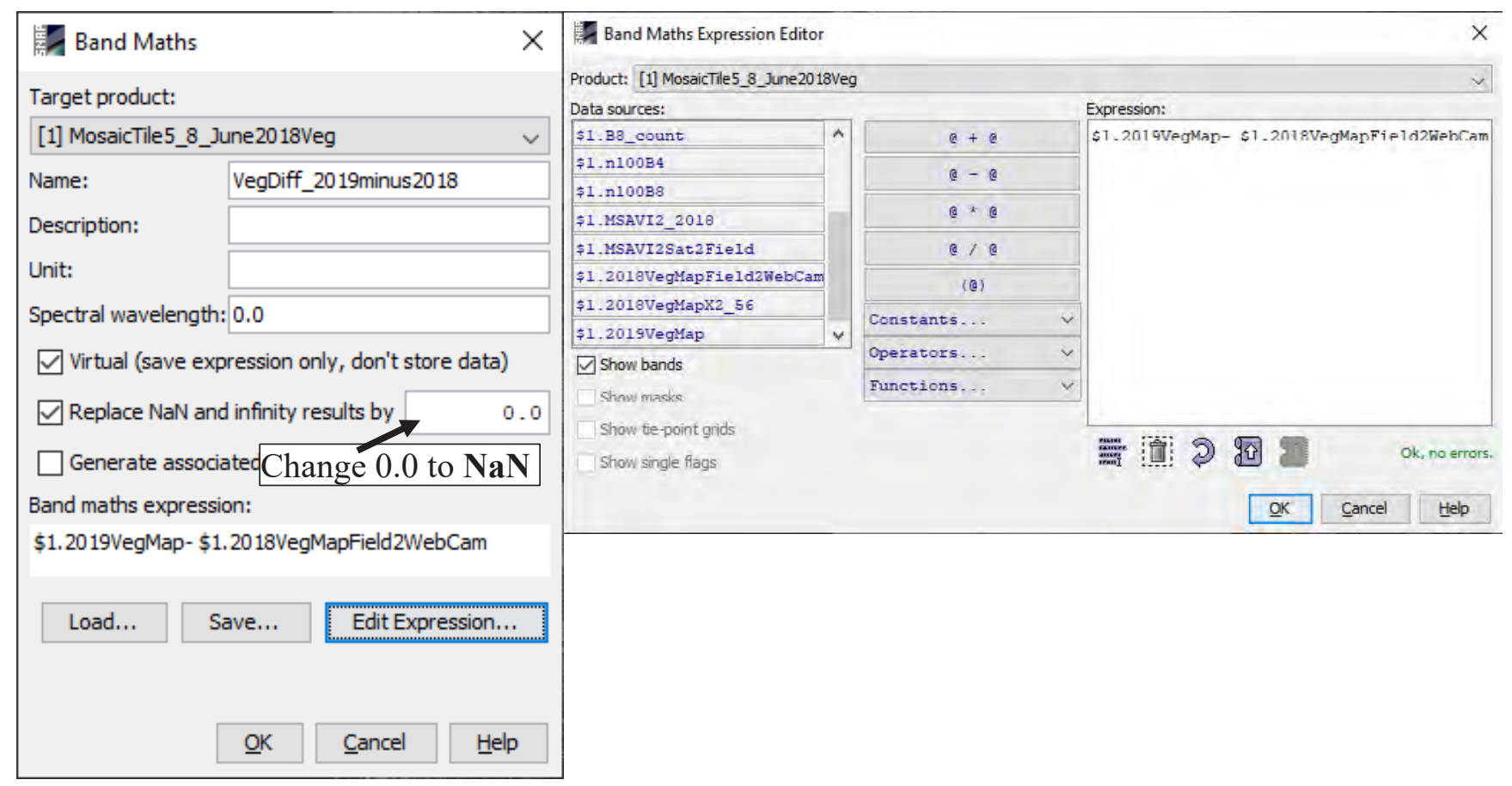




\section{C. Transforming the decimal VegDiff image to an integer format.}

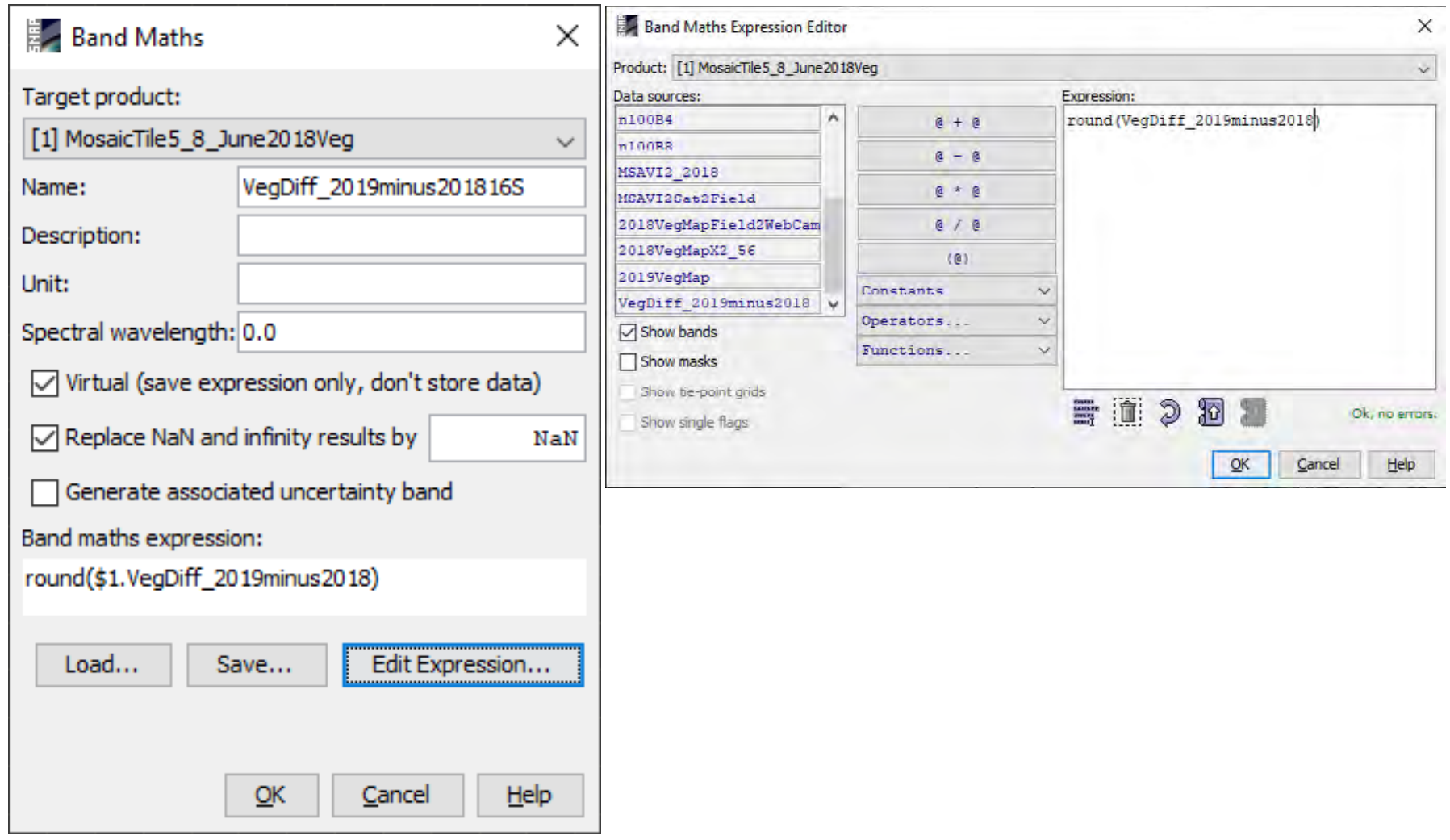

\section{D. Limit the upper VegDiff to 100 percent.}

The actual upper limit of the difference was 113 and the lower limit -114 . The upper limit is cut off at 100 percent LFC difference.

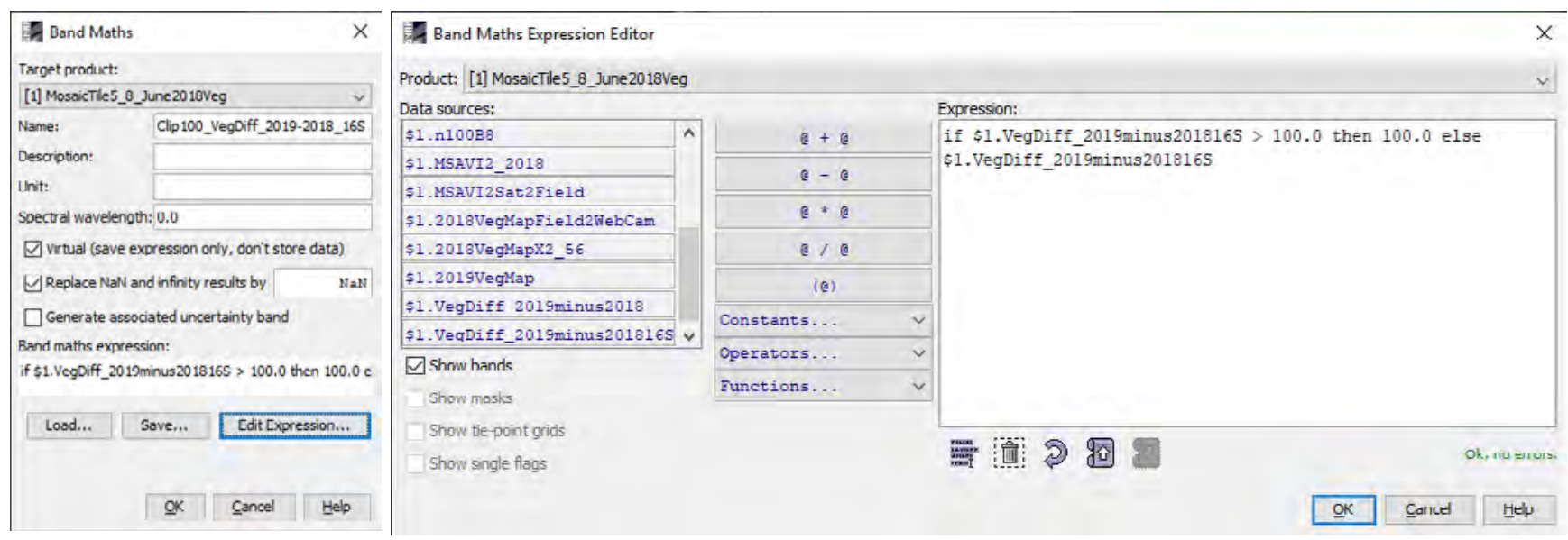

Small differences (1-2 percent) were noticed between the VegDiff map created in SNAP and the same VegDiff map created in PCI Geomatica, another image processing software. The difference arises because SNAP processes all image data as real, while other software provide signed and unsigned 16bit processing. 


\section{E. Scale the $16 S$ image from 0 to 100 to 0 to 255 .}

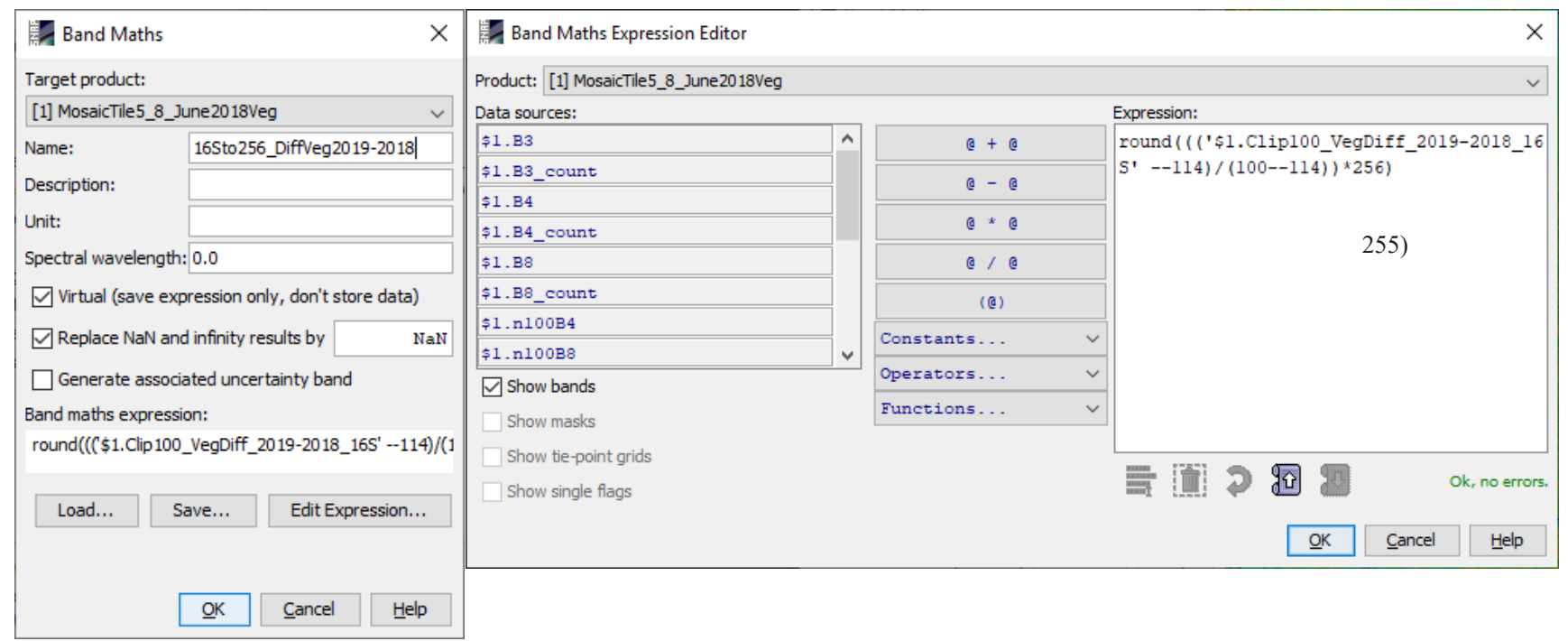

Scale the image using the min and $\max -114$ and 100 to 0 to 255 (16Sto255_DiffVeg2019-2018). See Band Maths expression above [((B3--114)/(100--114) $* 255]$. B3 is the 16S image data input per pixel and -114 and 100 are the $16 \mathrm{~S}$ min and max. The equation converts the $16 \mathrm{~S}$ image values from 0 to 1 so multiplication by 255 outputs an image with a $0-255$ range.

\section{F. Convert the $16 S$ band to $8 \mathrm{U}$ numerical format.}

Menu $>$ Raster $>$ Data Conversion $>$ Convert Datatype.

Convert Datatype creates a separate product with a *.Cnv extension.

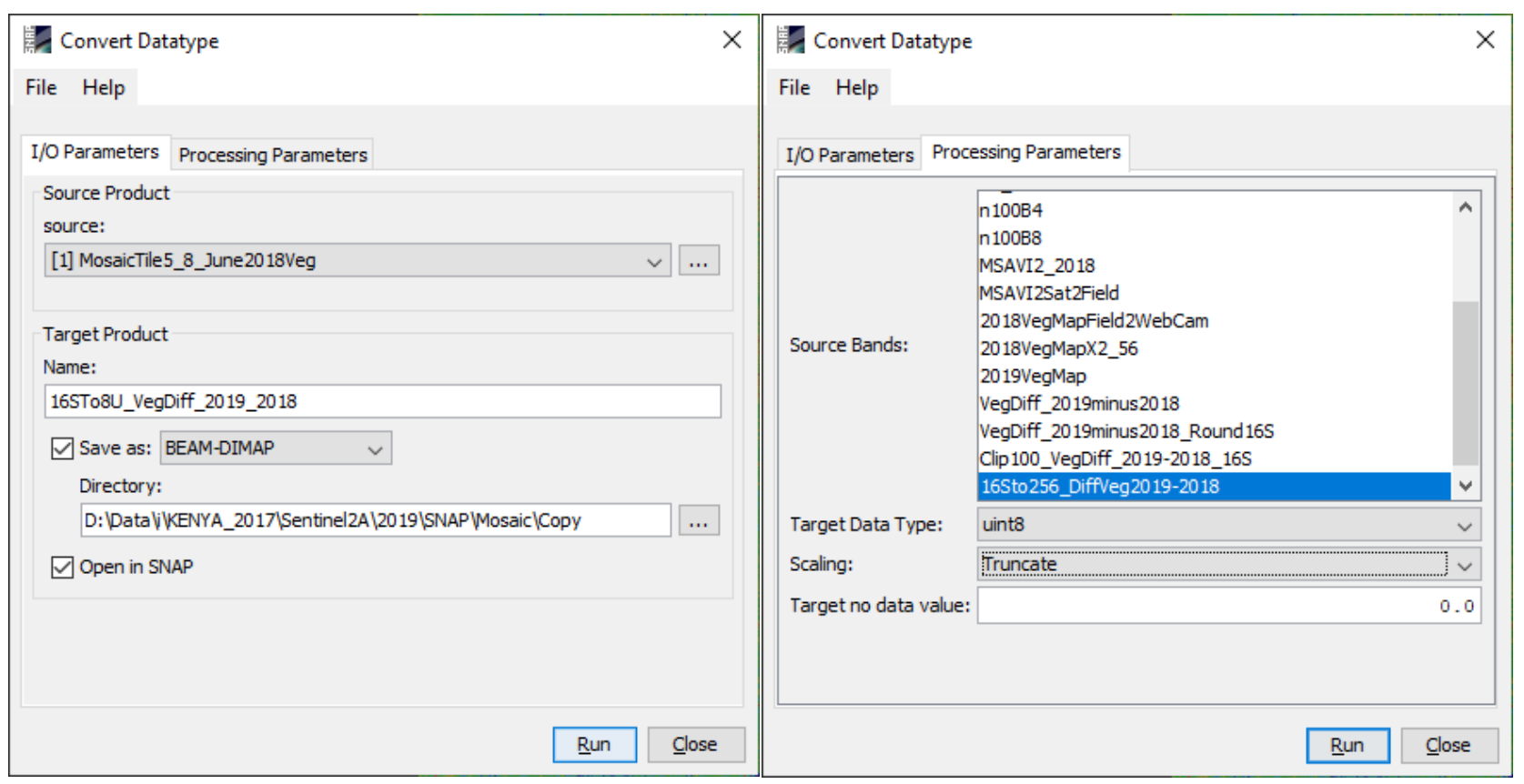

Although rounded as shown in $6 \mathrm{E}$, the $16 \mathrm{~S}$ image is still in a float or real format. The SNAP Convert Datatype outputs the data as a 0-255 8-bit unsigned image. The conversion to the $8 \mathrm{U}$ from the $16 \mathrm{~S}$ format provides a less complex data structure for color renditions and GIS analyses. 


\section{Export of the black and white VegDiff map.}

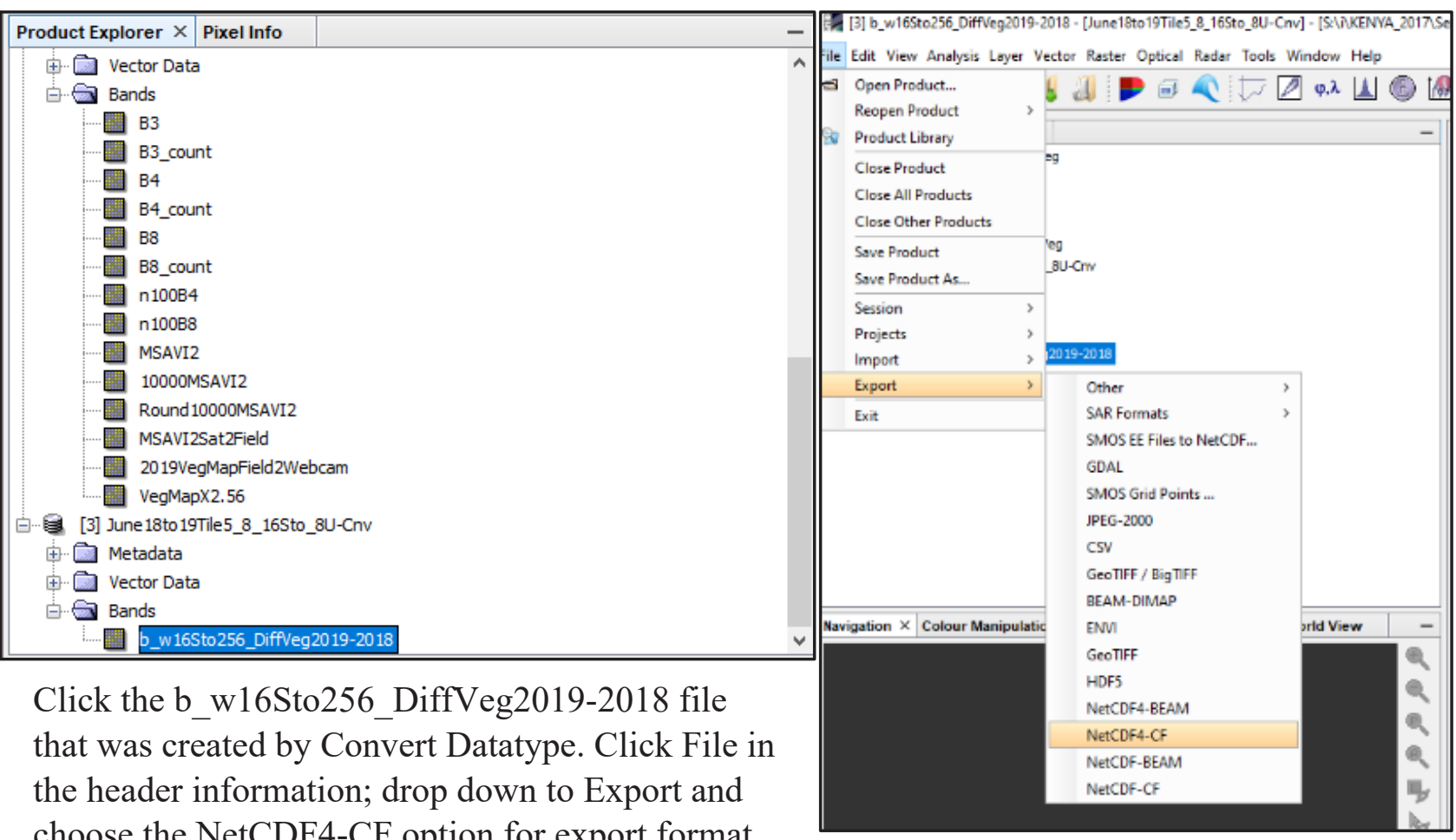

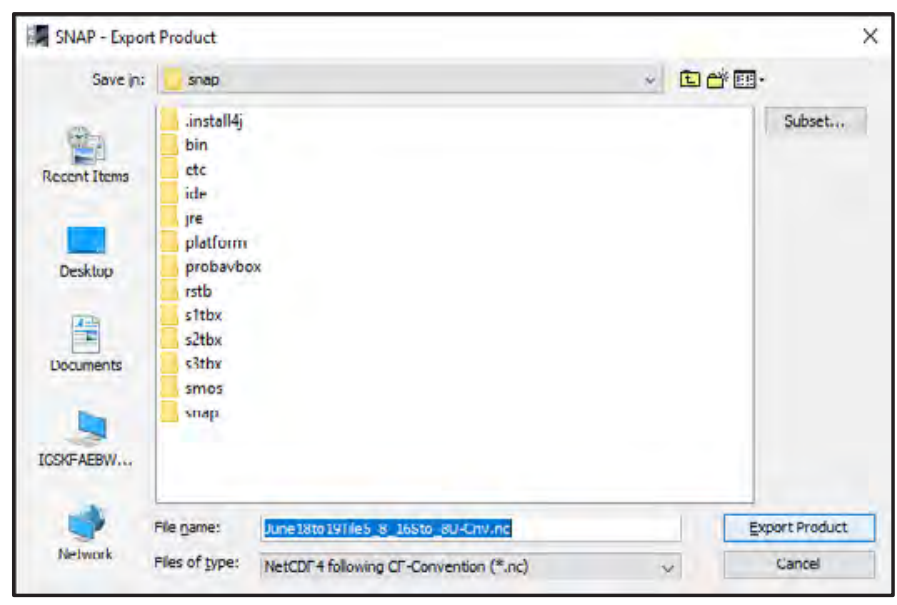

Navigate to Directory to store exported files. SNAP will create a directory at that location with the SNAP project name and the *.nc.data extension.

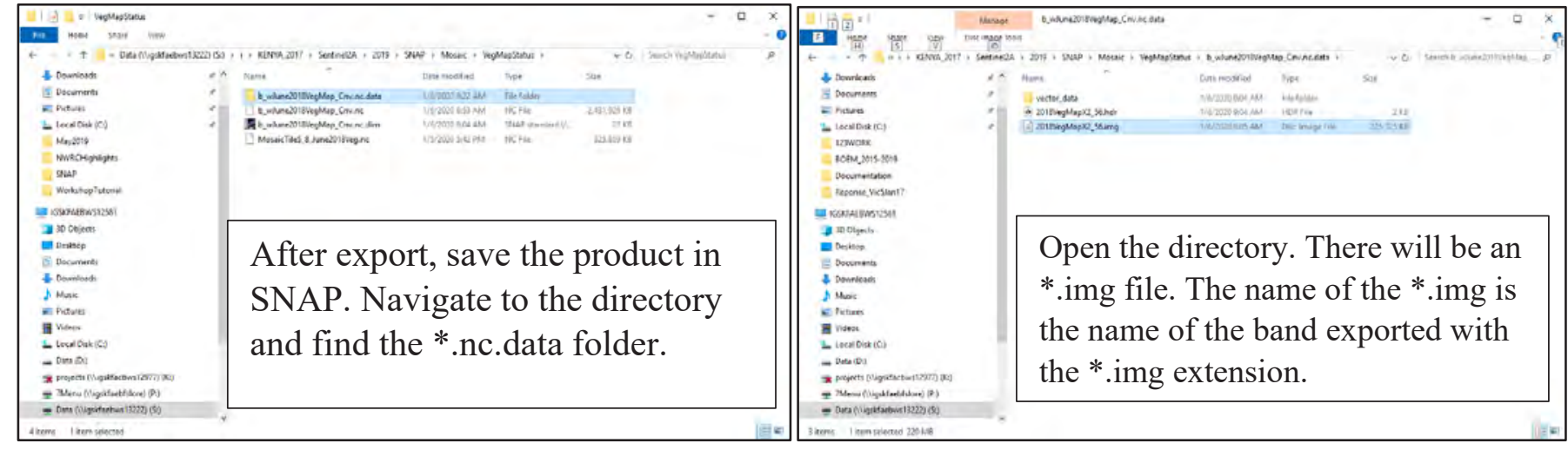




\section{Create a color ramp in SNAP for the LFC status and change maps.}

The color rendering of the LFC maps in SNAP provides a first check on the created products. To provide that assessment, we will develop and apply a color ramp to LFC status and difference maps.

\section{A. Creating a color ramp in SNAP.}

View the black and white target image in SNAP. Click Color Manipulation as shown below. Click on 100 percent in the set of icons to the right of the histogram. To add sliders, right click on the black and white color bar below the histogram and an Add New Slider message box appears. Add slider.

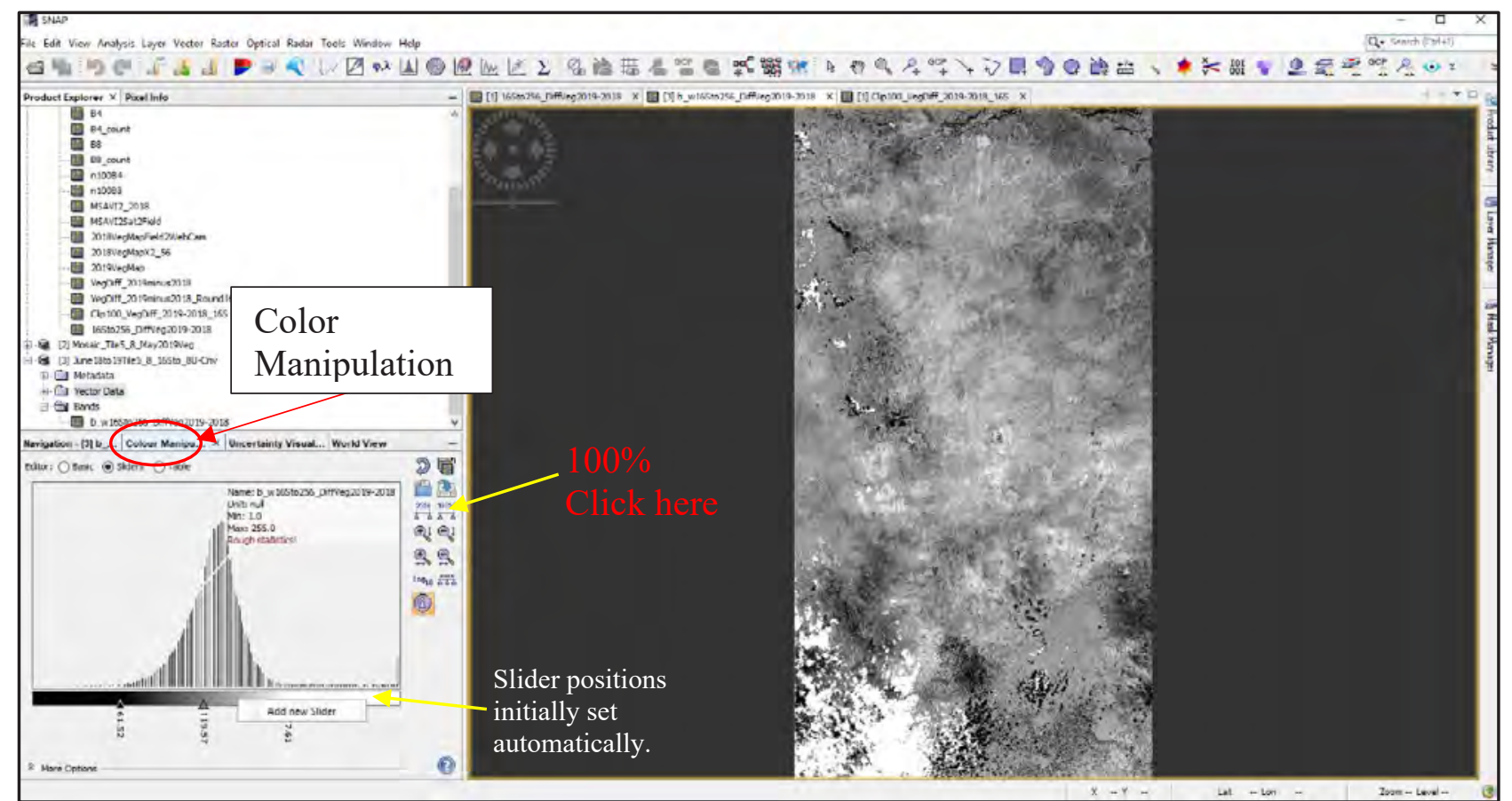

A color ramp was customized for the vegetation status maps; the upper and lower sliders (breakpoints) were defined to keep a uniform color lookup table.

Next, set slider color by clicking on the slider triangle; click RGB on the popup Select Color dialogue box. Set the Red, Green and Blue color values or use color code. Click OK.

For Vegetation Status Map: Set Color for lower limit RGB 139, 69, 19; color code \# 8b4513.

Set Color for upper limit RGB 154,

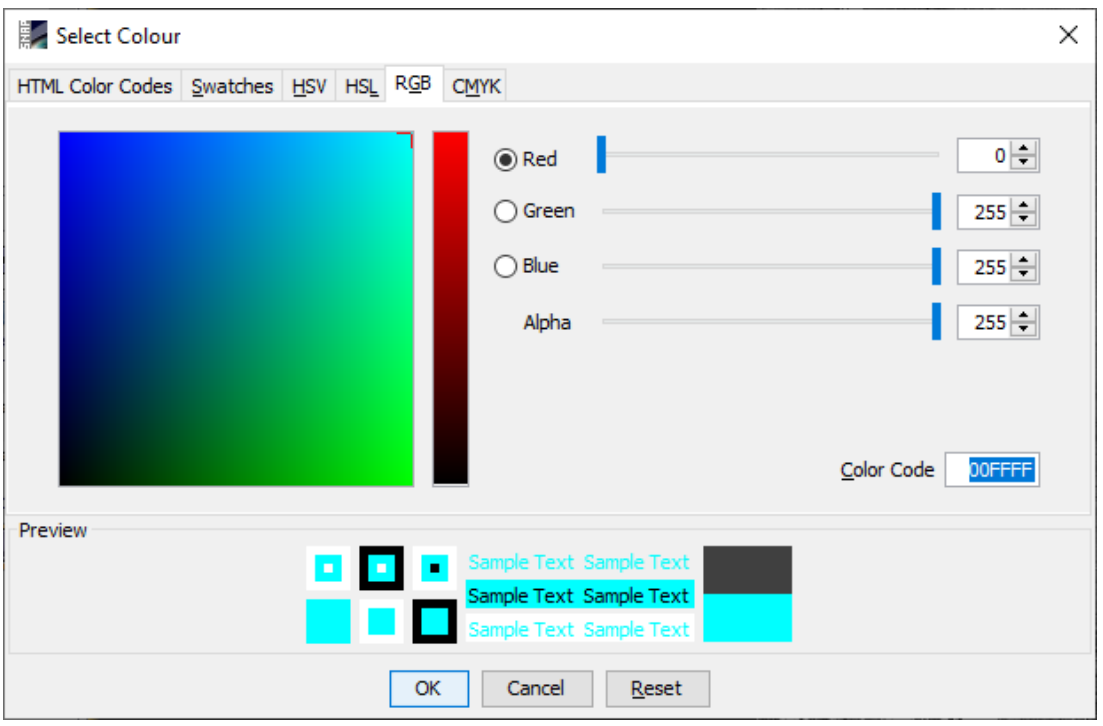
205, 50; color code \# 9acd32. 
The example below shows the slider color changed after clicking OK on the screen capture shown above. The box shown to the right of the slider (highlighted) contains the present slider position on the color ramp. Change this number to position the slider to breakpoints as shown in $8 \mathrm{~B}$.

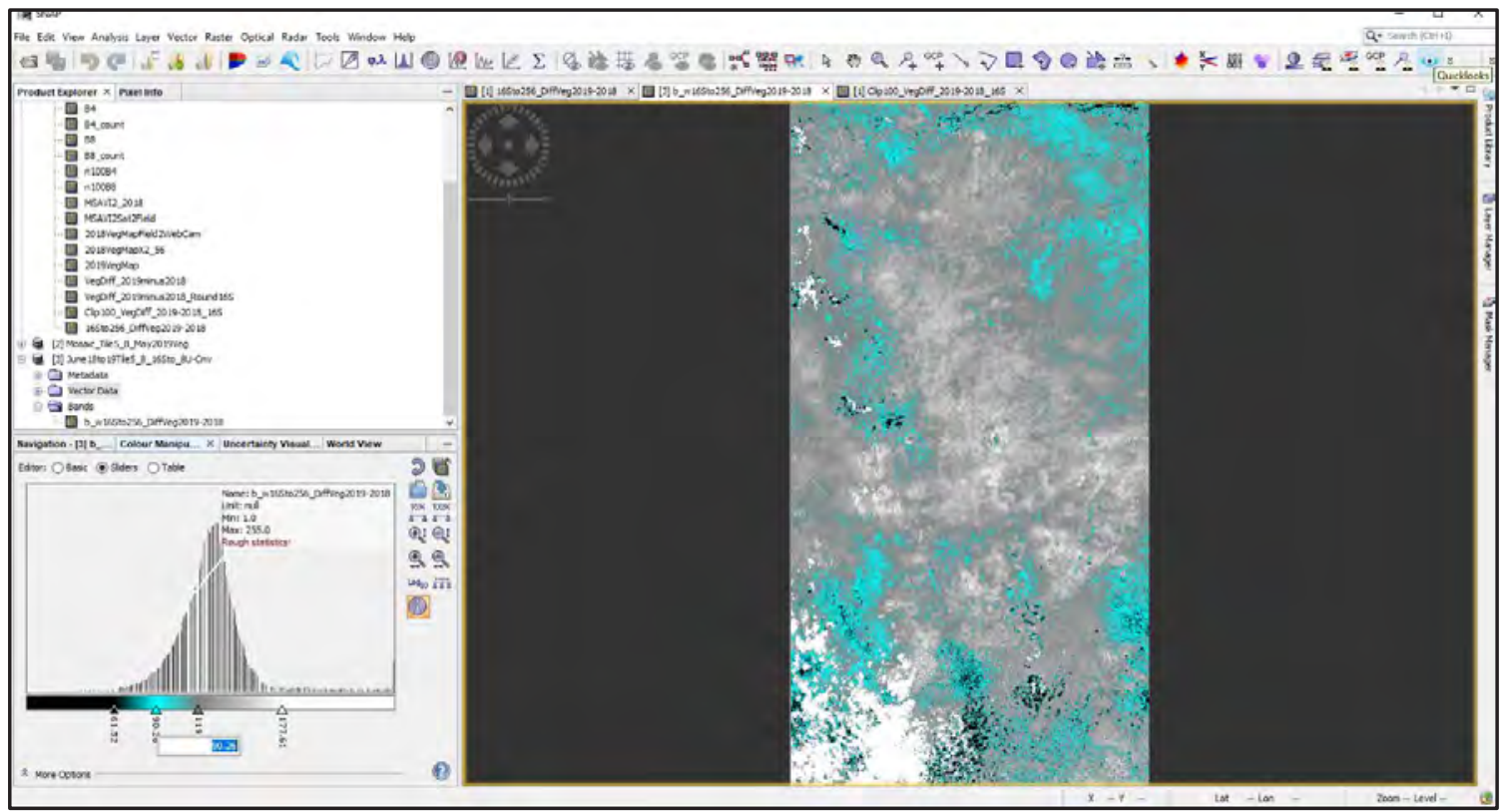

8B. Convert the 0-100 percent breakpoints to 0-255 percent breakpoints (two-tile breakpoints).

\begin{tabular}{|c|c|c|c|c|c|c|}
\hline Colors & 0-100 Break Points & 0-255 Break Points & Red & Green & Blue & Hexadecimal \\
\hline Blue & -44.0 & 83.41 & 0 & 0 & 255 & $0000 \mathrm{ff}$ \\
\hline Cyan & -26.35 & 104.44 & 0 & 255 & 255 & $00 \mathrm{ffff}$ \\
\hline Green & -8.35 & 125.89 & 0 & 255 & 0 & $00 \mathrm{ff00}$ \\
\hline Yellow & 9.65 & 147.34 & 255 & 255 & 0 & $\mathrm{ffff00}$ \\
\hline Red & 27.65 & 168.79 & 255 & 0 & 15 & $\mathrm{ff000f}$ \\
\hline Pink & 45.29 & 189.81 & 255 & 160 & 255 & $\mathrm{ffaOff}$ \\
\hline
\end{tabular}

Convert the $16 \mathrm{~S}$ breakpoints to $8 \mathrm{U}$ breakpoints in Excel (recommended), if available (or an open source workbook), for example $((-44--114) /(100--14)) * 255=83.41$. 


\section{Color renditions across raster files with different data types.}

The examples below created in SNAP show the different color renditions when applying breakpoints from a $16 \mathrm{~S}$ color rendition to the same image converted to an $8 \mathrm{U}$ data type.

\section{A. Application of real data format (32-bit or 16-bit) breakpoints to $8 \mathrm{U}$ image data format.}

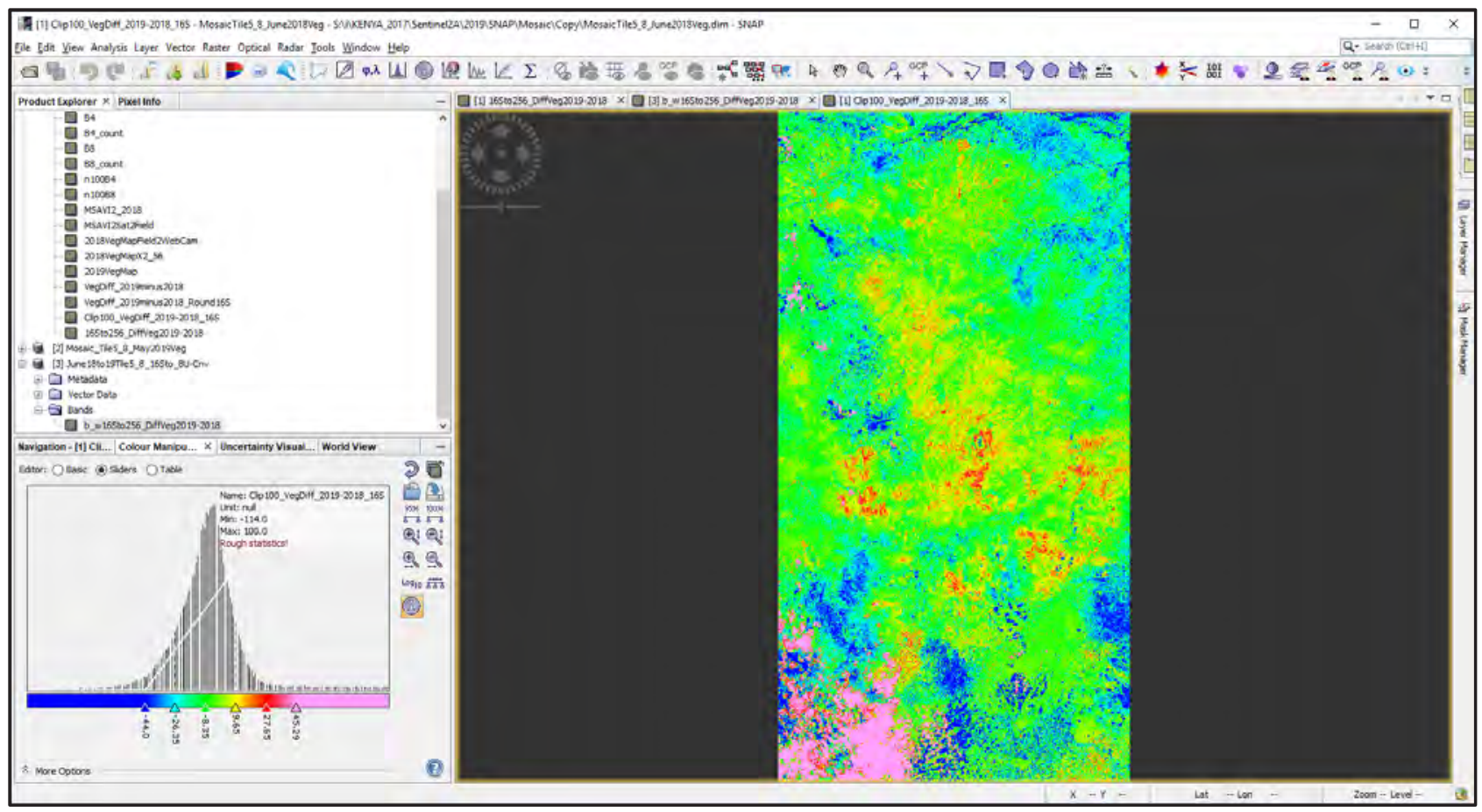

9B. Application of the $8 \mathrm{U}$ breakpoints to the $8 \mathrm{U}$ image data format.

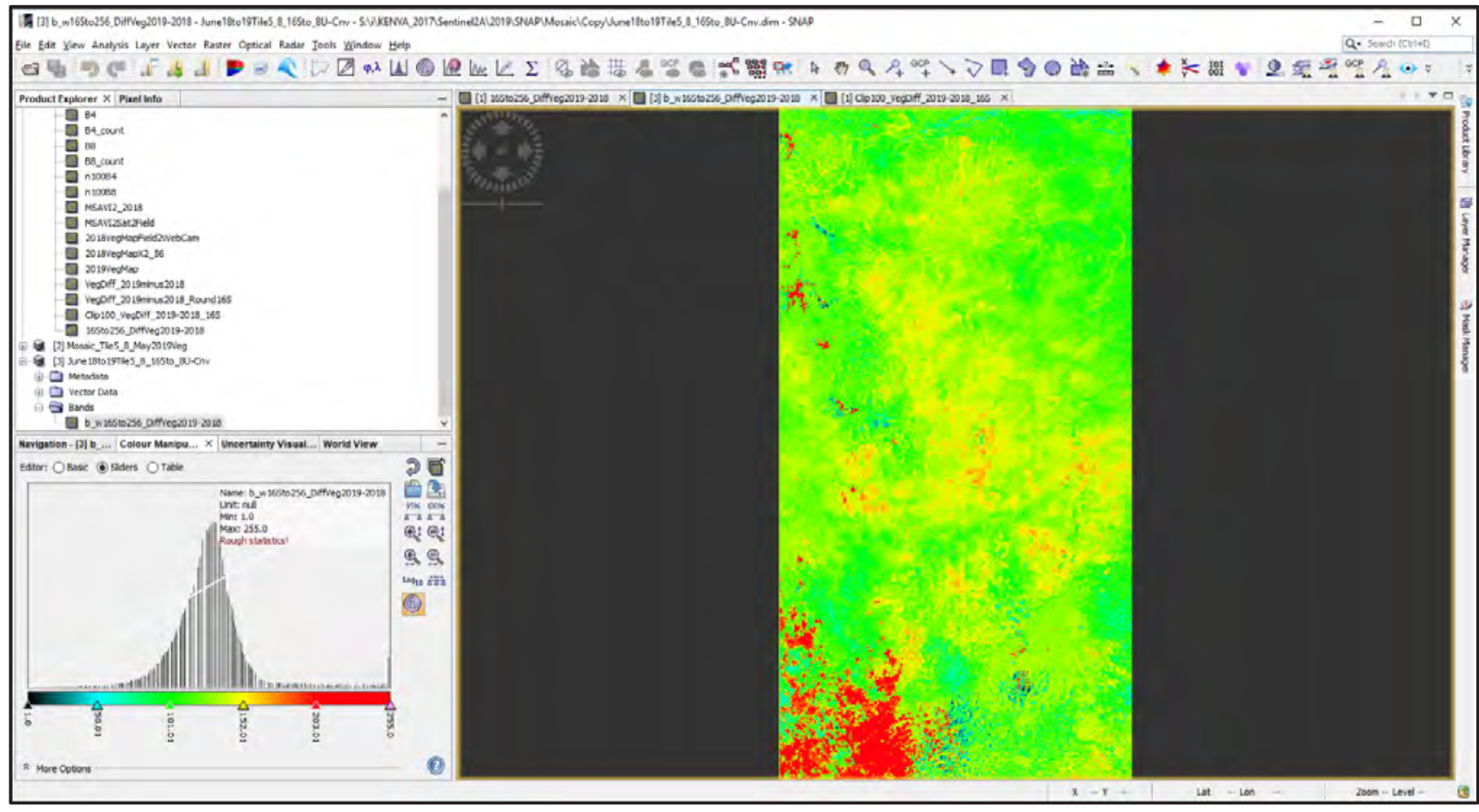


Notes on applying color ramp in SNAP and QGIS: To retain the color rendering from image to image and from software platform to platform, the same breakpoints must be set at the same positions and colors. If applying a color rendition taken from a $16 \mathrm{~S}$ data format to one with an $8 \mathrm{U}$ format, prescaling of the data and breakpoints is necessary. Even with scaling, application of color rendering from one data format (source) to another (target) may require adjustment of the target range to get the rendition of the target to exactly mimic the look of the source rendition. 


\section{Atmospheric correction of Sentinel-2 images using ESA software.}

Note: The ESA hub does not have all scenes atmospherically corrected (MSIL2A). Therefore, MSIL1C files must be atmospherically corrected using a DOS-based software called Sen2Cor from ESA.

\section{A. Install Sen2Cor (SNAP atmospheric correction software).}

Go to http://step.esa.int/main/snap-supported-plugins/sen2cor/.

Save Sen2Cor to the Program directory.

Open the DOS cmd window.

$\mathrm{C}: 1>\mathrm{cd}$ data

C: $:$ Data $>$ cd Sen2Cor

$\mathrm{C}: \backslash$ Data $\backslash$ Sen2Cor $>$ cd Sen2Cor-2.4.0-win64

C: $\backslash$ Data $\backslash$ Sen2Cor $\backslash$ Sen2Cor-2.4.0-win64>L2A_Process.bat -help

\section{B. The atmospheric correction process.}

Note: Run the full atmospheric process (that is, L2A_Process) for all resolutions as the cloud mask and land classification are created for 20-m resolution. The command in CMD window for L2A_Process is C:IData $\backslash$ Sen2Cor $\backslash$ Sen2Cor-2.4.0-win64\L2A_Process D:|Datali|KENYA_2017 $\backslash$ Sentinel2A $\backslash J u n e 2017 \backslash$ Tile 5\Sen2Cor 2 A_MSIL1C_20170628T075211_N0205_R092_T37NCB_20170628T075749.SAFE Note: L2A_Process will process all spatial resolution images and all ESA produced ancillary products.

Note: The atmospherically corrected output file will have a similar name, except that MSIL1C is changed to MSIL2A created output file name listed below:

S2A_MSIL2A_20170628T075211_N0205_R092_T37NCB_20170628T075749.SAFE

Arrow shows the command typed to execute the L2A_Process.

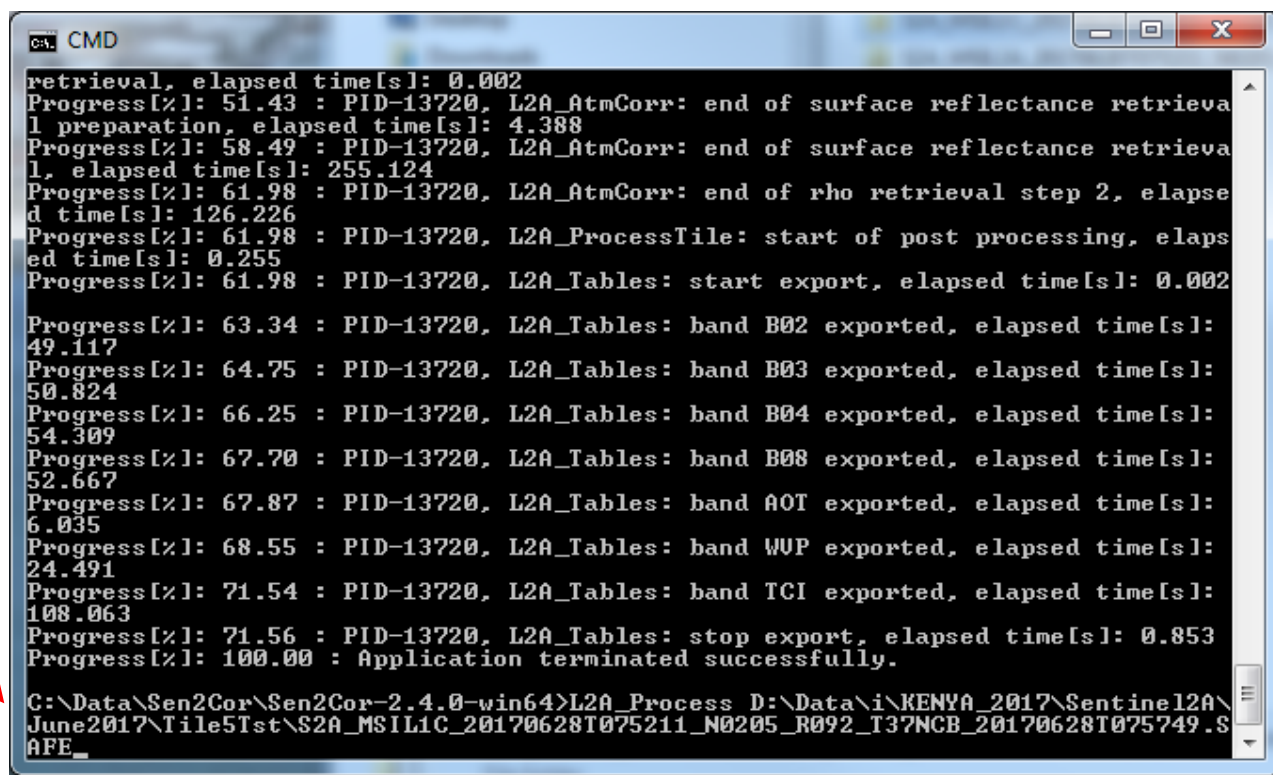




\section{Synthetic Aperture Radar (SAR) image data processing —Sentinel 1}




\section{Sentinel-1A SAR image data.}

\section{A. Opening the SAR image in SNAP.}

After downloading the SAR images, unzip the download folders and Import in SNAP the *.manifest.safe file.

\section{B. Calibrate the SAR image data to sigma naught.}

Menu $>$ Radar $>$ Radiometric $>$ Calibrate. Next, set I/O Parameters and Processing Parameters in the Calibration dialog box. In I/O Parameters, create a new output directory for the SNAP output files. Enter that folder name under Target Product $>$ Directory and the product filename under Name. In Processing Parameters, select both VH and VV Polarisations and click Output sigma0 band.

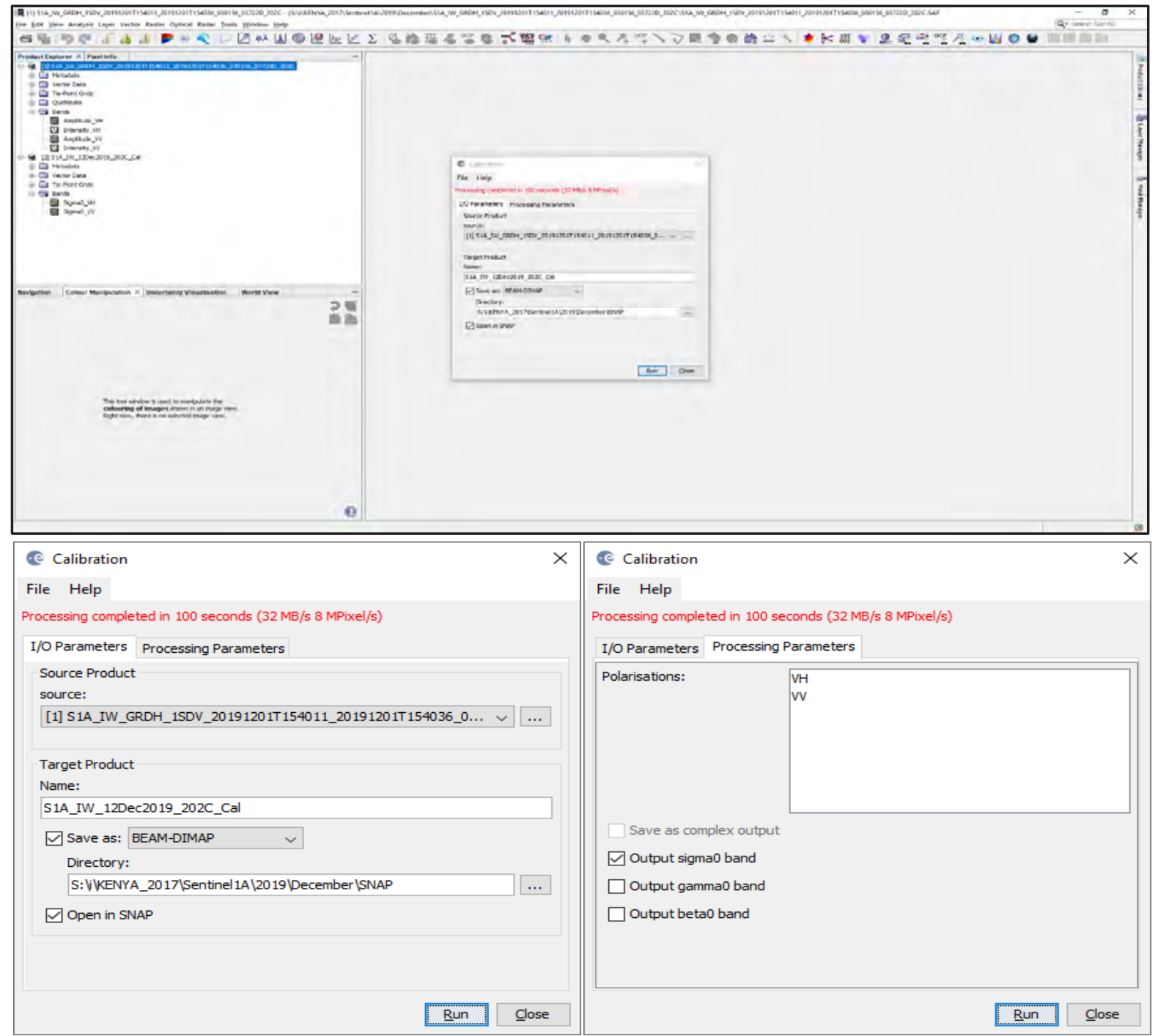

SNAP creates a pointer file *.dim that is saved in the output directory containing the calibrated image data.

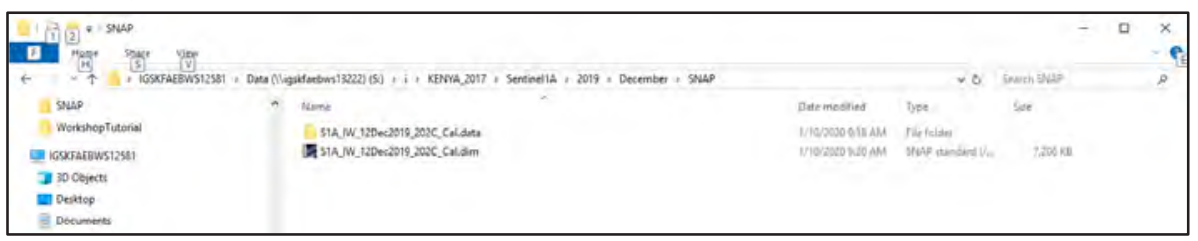




\section{C. Perform terrain correction and spatial resampling of the calibrated SAR image.}

Go to Menu $>$ Radar $>$ Geometric $>$ Terrain correction $>$ Range-Doppler.

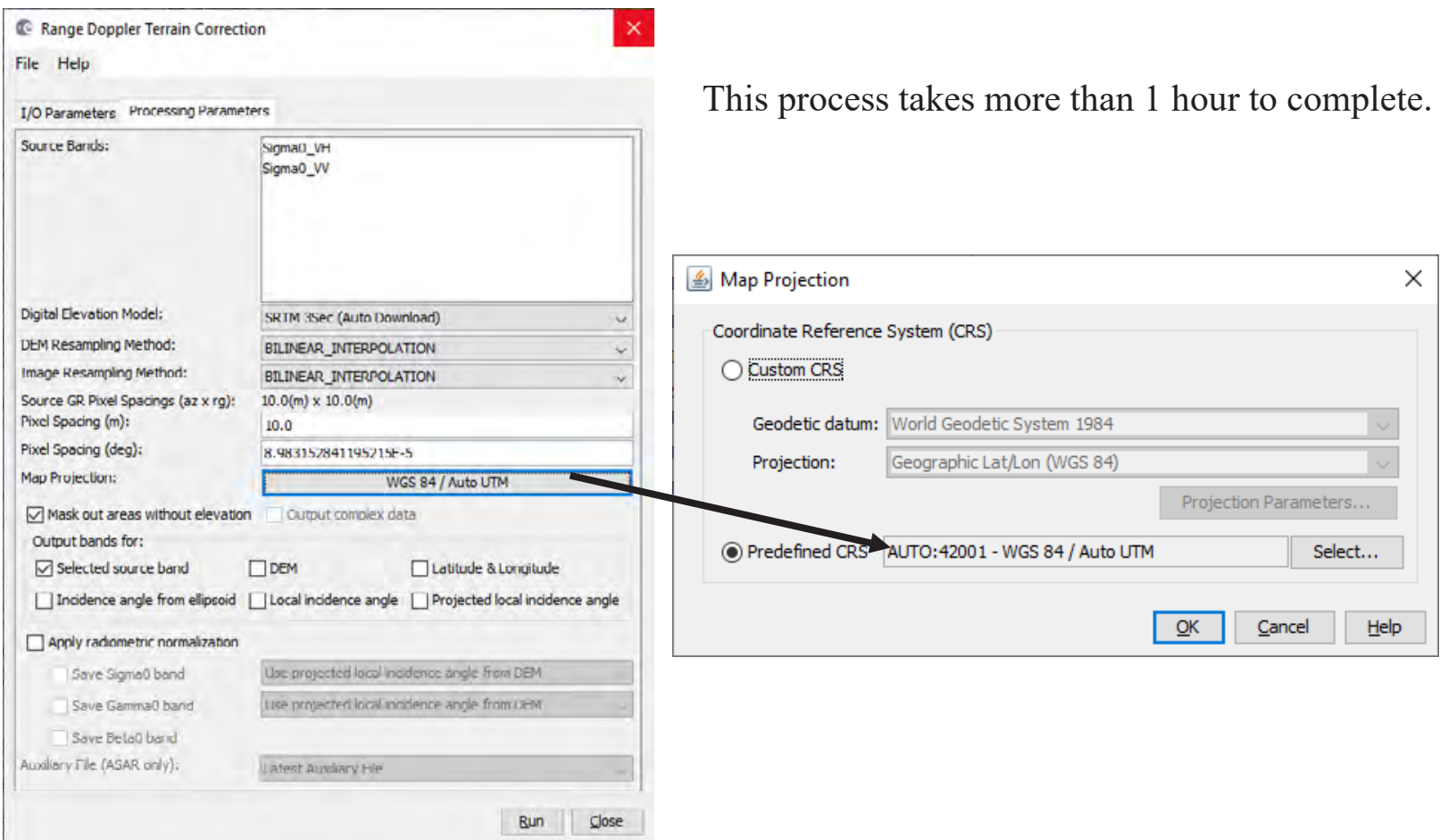

\section{D. Perform Speckle Filtering of the Calibrated and Terrain Corrected SAR image.}

Menu $>$ Radar $>$ Speckle Filtering $>$ Single Product Speckle Filter.
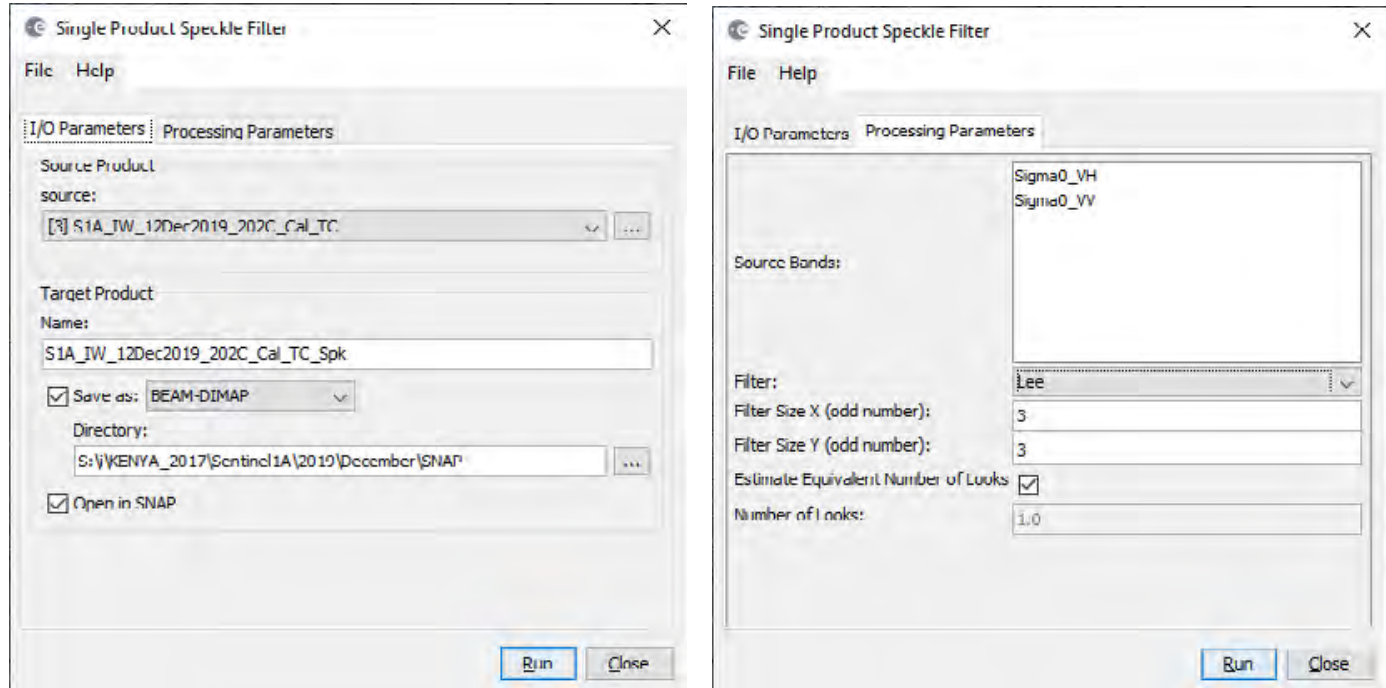


\section{Create mosaic of the four radar scenes processed with speckle filter.}

(for example, S1A_IW_6Dec2019_1064_Cal_TC_Spk.dim)

Menu $>$ Radar $>$ Geometric $>$ SAR-Mosaic.

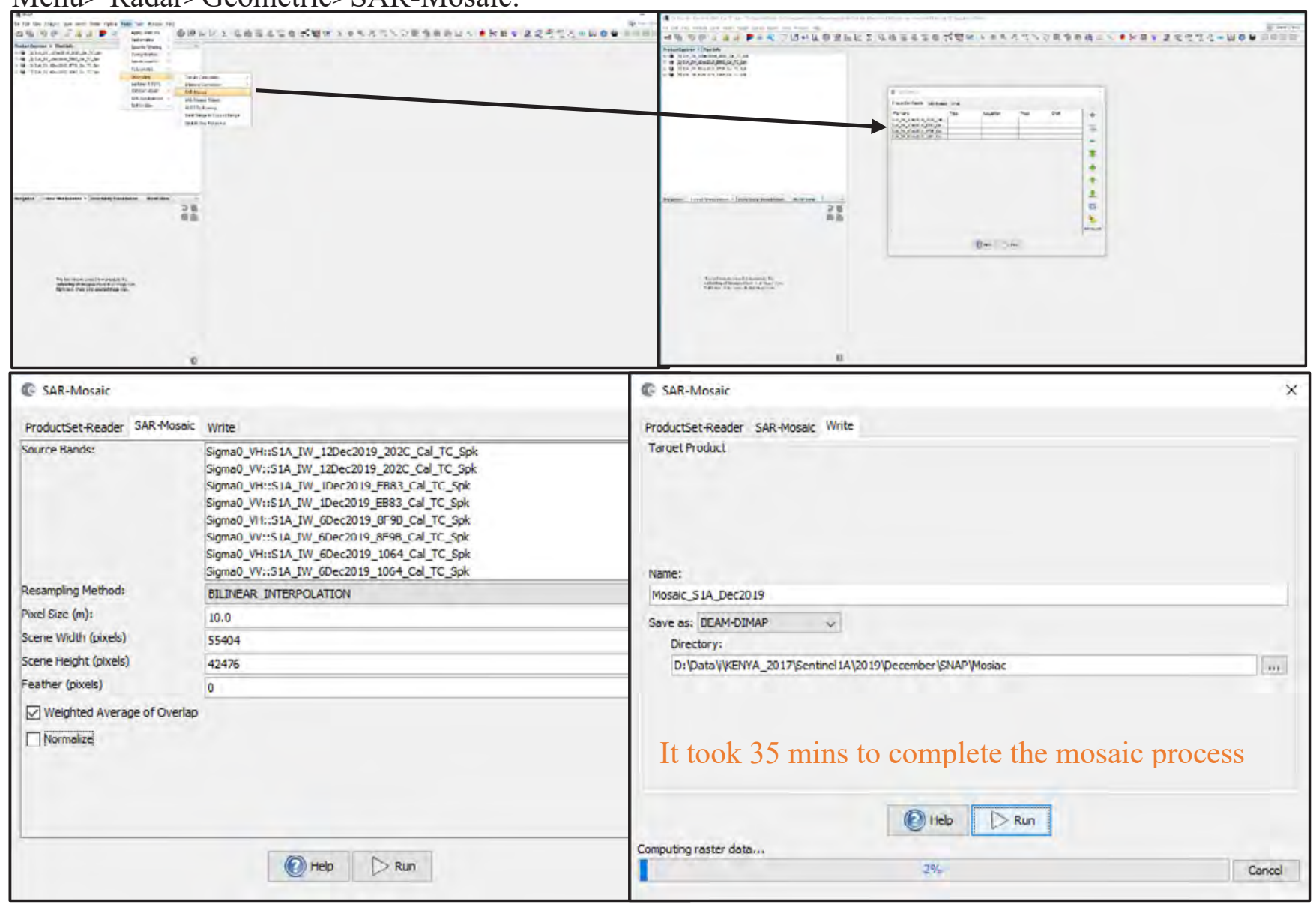

To view the mosaic, double click on one of the bands to view it. Because it is a large file, it will take about 10 minutes to bring up the image.

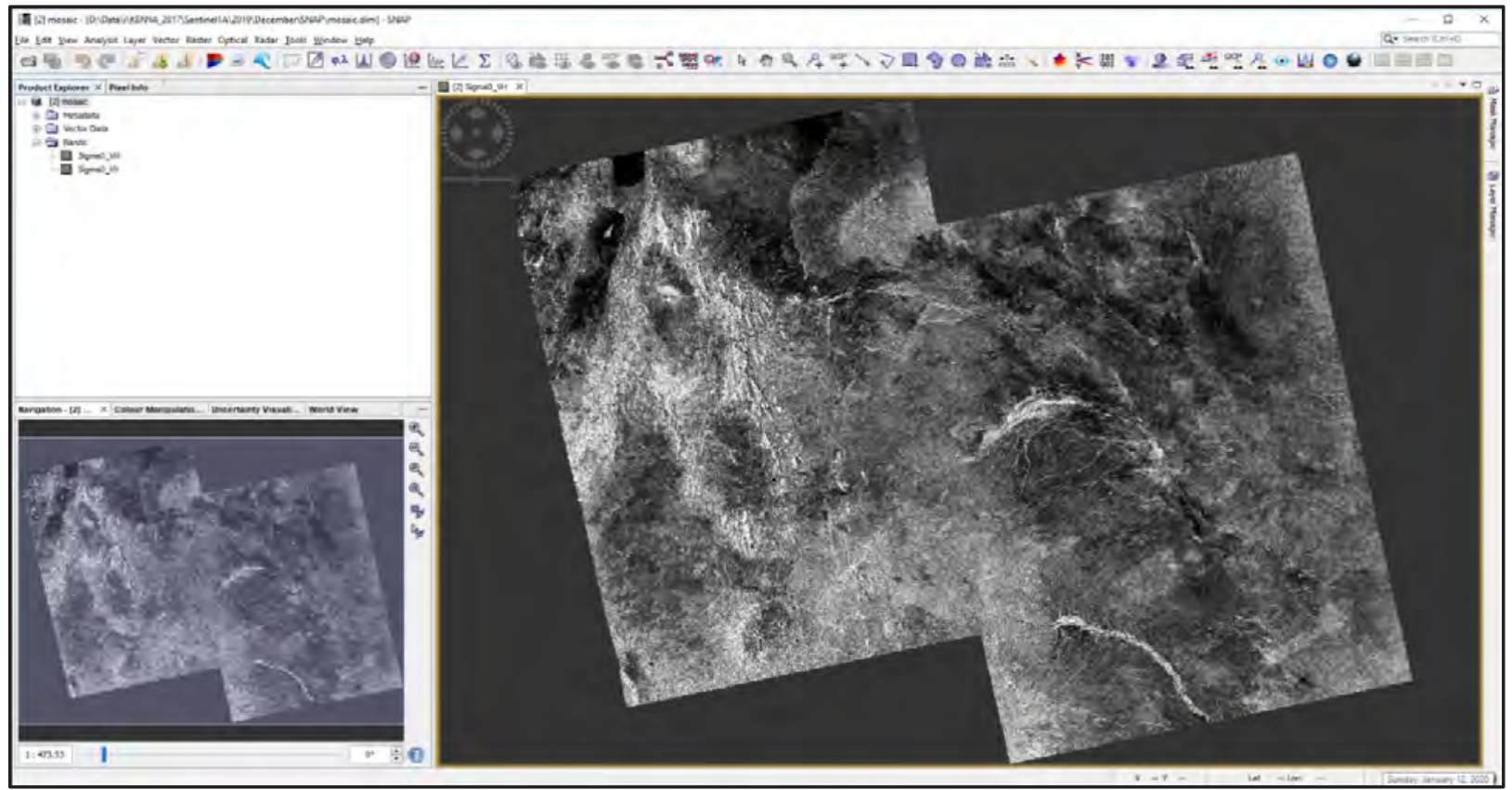




\section{Pixel value extractions.}

\section{A. Input Image data file.}

Working on the same mosaic file created on preceding page (section 2, page 22).

Menu $>$ Raster $>$ Export $>$ Extract Pixel Values. In the dialog window Pixel Extraction under Input/Output tab (screen capture 1), Source Path is defined as the mosaic image that is already opened in SNAP; specify the path of the output directory and a prefix for the output filename. Next, click the Parameters tab and use the parameters specified in screen capture 2.
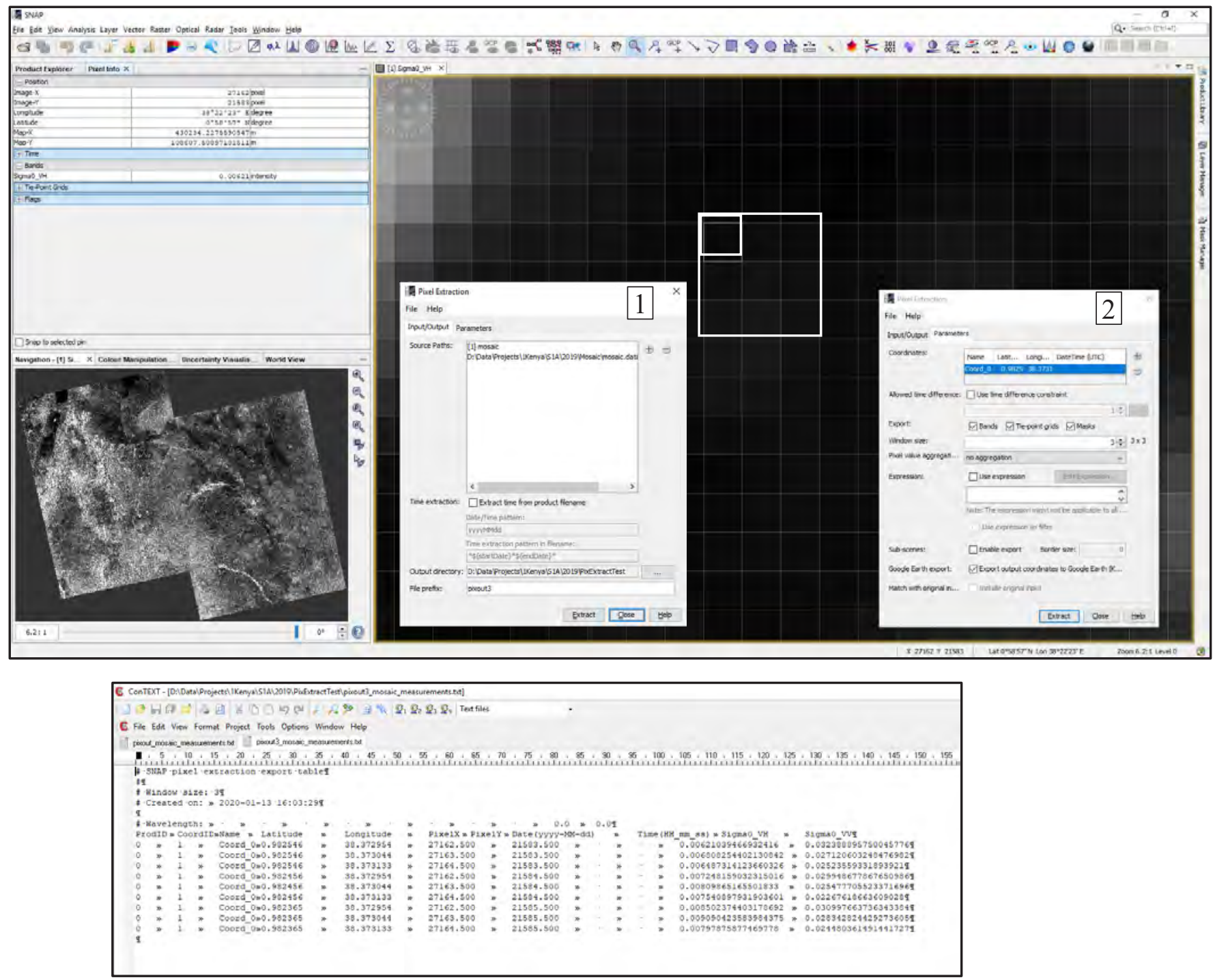

The output for a $3 \mathrm{X} 3$ window is shown above. The upper left pixel in the $3 \mathrm{X} 3$ window is highlighted in the graphic. Check the highlighted pixel position value in the image and the top value (1 of 9) in the output table shown above. Sigma VH and sigma VV should match in the table and in the image. 


\section{B. Input coordinate file.}

Create an Excel data sheet following the format shown on the Excel sheet below. Save the Excel file as a Text tab delimited (*.txt) file. The text input with latitude/longitude coordinates can be input in the Pixel Extraction Parameters dialogue box shown on right. Click on the + icon in the Coordinates section and select Add coordinates from file ...; navigate to the saved tab delimited text file and load file into the Coordinates dialogue box as shown below on the right.

Menu $>$ Raster $>$ Export $>$ Extract Pixel Values.

Fill out Extract Pixel Values dialogue boxes as shown below and click on Extract.

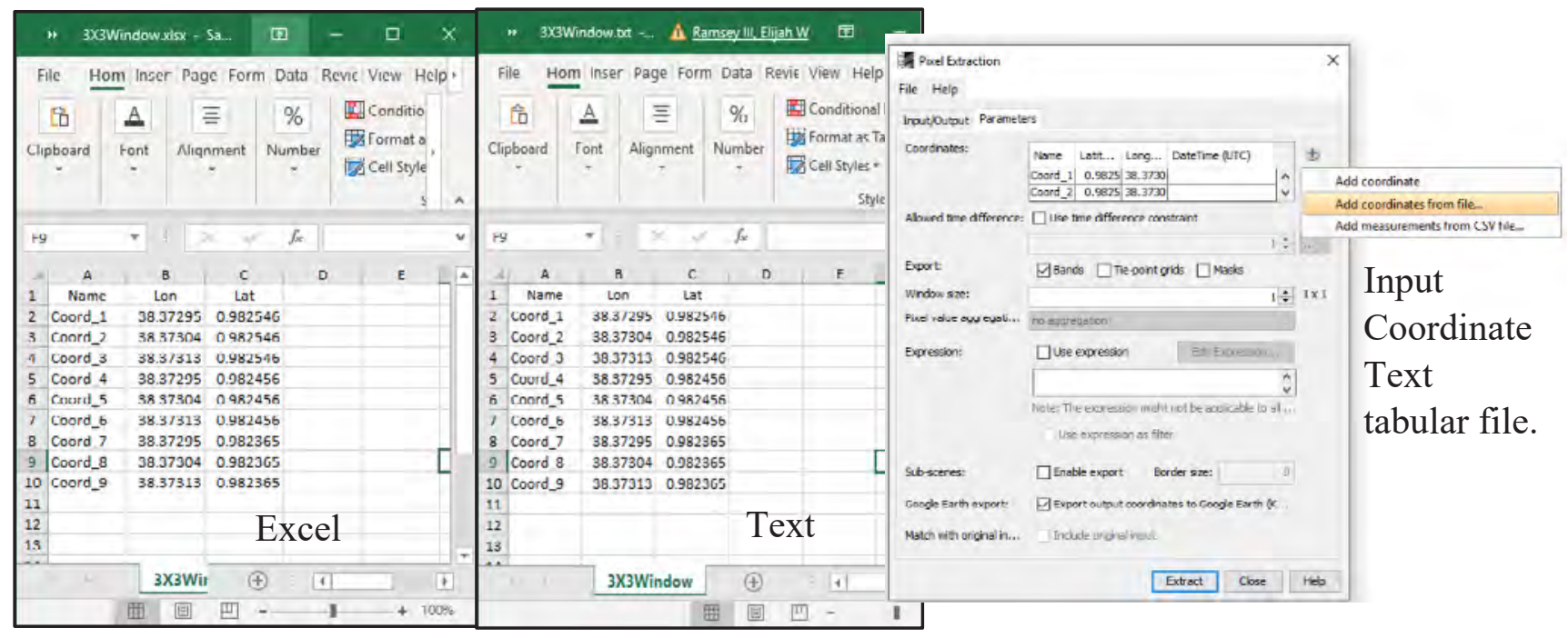




\section{Create a ratio band VH/VV.}

The VH return is a mixed polarization return of the VV send amplitude. The vegetation normally is typified as a mixed orientation of elements (plant leaves and stalks) that scatter a portion of the like (linear) polarizations (VV) into nonvertical or horizontal orientations, typified as VH. The ratio indicates vegetation component of scatter.

Band Maths
Target product:
Name:
Description:
Unit:
Spectral wavelength: 0.0
$\square$ Virtual (save expression only, don't store data)
$\square$ Replace NaN and infinity results by
$\square$ Generate associated uncertainty band
Band maths expression:

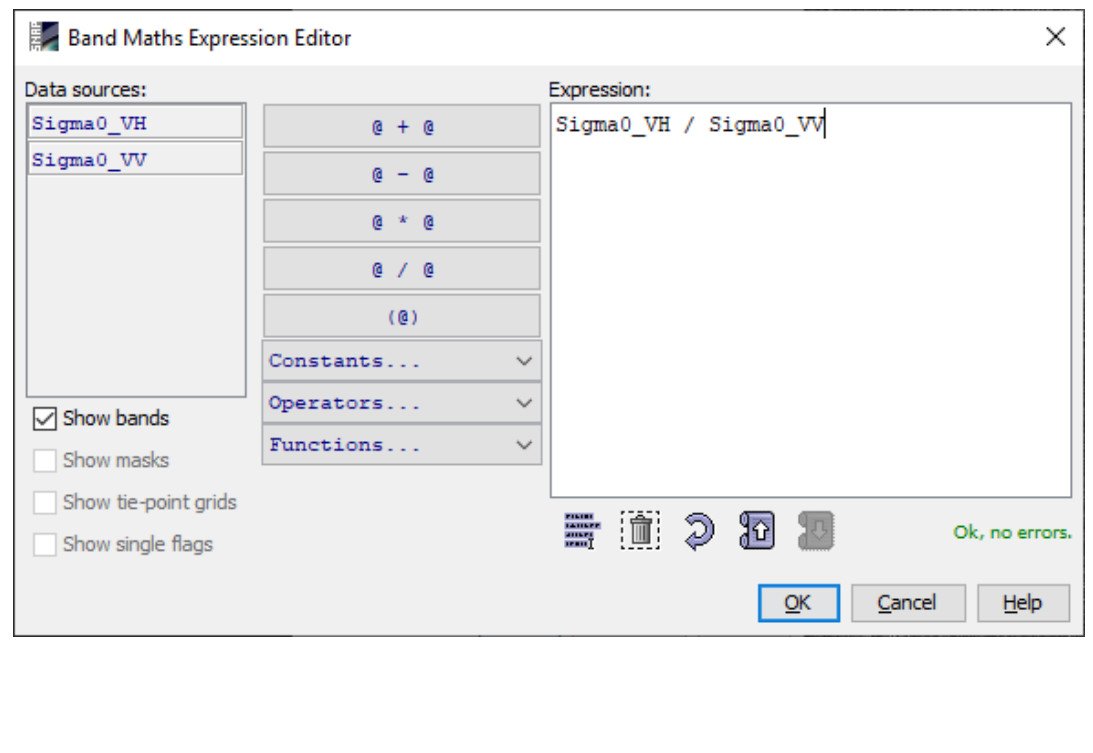

\section{A. Using SAR data as an indicator of vegetation density.}

- $\quad$ Load the 2019 SAR image mosaic of core-NRT.

- Use the RGB function to create a color rendition of the VV and VH SAR bands. Put VH in the red and green color band and the VV in the blue color band.

- Apply the leaf area index (LAI, density indictor) calibration to the SAR VH/VV ratio image. $\mathrm{LAI}=6.02 * \mathrm{VH} / \mathrm{VV}-0.40$. (This equation is provided in the OFR, page 82, figure 42; https://pubs.er.usgs.gov/publication/ofr20191037).

- The upper limit of the calibration equation is 3.2 LAI. Use SNAP tools to exclude mapped LAI values greater than 3.2. 


\section{Create a subset to match the subset of the 2018 S1A mosaic.}

This section illustrates the steps required to create a subset; how to note the coordinates of the reference image (here subset $2018 \mathrm{~S} 1 \mathrm{~A}$ mosaic); and how to use these for subsetting the $2019 \mathrm{~S} 1 \mathrm{~A}$ mosaic image. To note the geocoordinates of the 2018 subset, go to Menu>Analysis $>$ Geo-Coding. Note the upper right latitude, upper right longitude, lower left latitude, and lower left longitude. These will be used to make the subset. The dd mm ss must be converted to decimal degrees.

\begin{tabular}{|c|c|c|}
\hline 原 & & $\times$ \\
\hline Geo-Coding $\times$ & & \\
\hline Center latitude & $1^{\circ} 21^{\prime} 09^{\prime} \mathrm{N}$ & $\wedge$ \\
\hline Center longitude & $37^{\circ} 55^{\prime} 45^{\prime \prime} \mathrm{E}$ & \\
\hline Upper left latitude & $2^{\circ} 42^{\prime} 41^{\prime \prime} \mathrm{N}$ & \\
\hline Upper left longitude & $36^{\circ} 46^{\prime} 12^{\prime} \mathrm{E}$ & \\
\hline Upper right latitude & $2^{\circ} 42^{\prime} 48^{\prime} \mathrm{N}$ & \\
\hline Upper right longitude & $39^{\circ} 05^{\prime} 12^{n} \mathrm{E}$ & \\
\hline Lower left latitude & $0^{\circ} 00^{\prime} 28^{\prime \prime} \mathrm{S}$ & \\
\hline Lower left longitude & $36^{\circ} 46^{\prime} 21^{\prime} \mathrm{E}$ & \\
\hline Lower right latitude & $0^{\circ} 00^{\prime} 28^{\prime \prime} \mathrm{S}$ & \\
\hline Lower right longitude & $39^{\circ} 05^{\prime} 12^{\prime \prime} \mathrm{E}$ & \\
\hline & $\begin{array}{l}\text { FITTED_CS["Image CS based on WGS } 84 \text { / Auto UTM", } \\
\text { PARAM_MT["Affine", } \\
\text { PARAMETER["num_row", 3], } \\
\text { PARAMETER["num_col", 3], } \\
\text { PARAMETER["elt_0_0", 10.0], } \\
\text { PARAMETER["elt_0_2", 252069.22755905468], } \\
\text { PARAMETER["elt_1_1", }-10.0],\end{array}$ & $\checkmark$ \\
\hline$<$ & & 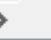 \\
\hline
\end{tabular}

Menu $>$ Raster $>$ Subset. In the Specify Product Subset dialog box, under

Spatial Subset north latitude=upper right latitude (from the Geo-Coding window above), west longitude $=$ lower left longitude, south latitude $=-$ lower left latitude, east longitude $=$ upper right longitude. Next, under Band Subset, select both Sigma0_VH, and Sigma0_VV, under Metadata Subset click Select all.
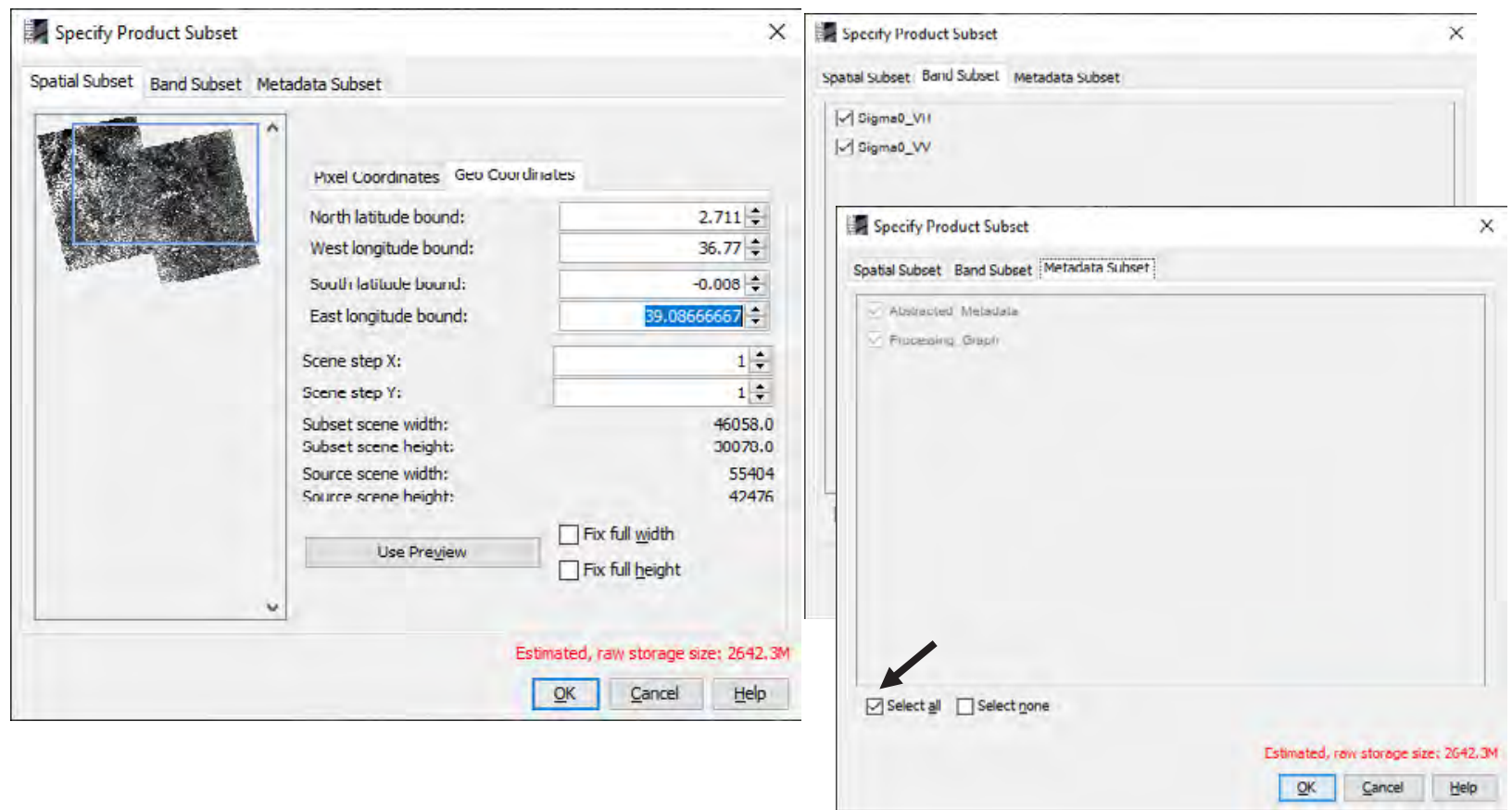


\section{References}

Qi, J., Chehbouni, A., Huete, A.R., Kerr, Y.H., and Sorooshian, S., 1994, A modified soil adjusted vegetation index: Remote Sensing of Environment, v. 48, no. 2, p. 119-126.

Rangoonwala, A, and Ramsey, E.W., III, 2019, Monitoring live vegetation in semiarid and arid rangeland environments with satellite remote sensing in northern Kenya: U.S. Geological Survey Open-File Report 2019-1037, 83 p. [Also available at https://pubs.er.usgs.gov/publication/ofr20191037.] 


\section{Section II-Geographic Information System Training Workshop}

\section{Quantum Geographic Information System (QGIS) Analysis of Grassland Live Fractional Cover Maps for Management of Wildlife and Pastoral Grazing}

This GIS workshop is designed for resource managers as a beginner level hands-on training course. The workshop demonstrates the application of basic procedures in GIS:

- Import, connect, and explore geographic information of various formats.

- Create and edit geographic information.

- Query, analyze, and present geographic information.

Objective:

- Although the procedures learned can be applied to GIS analyses of any raster or vector data, this course is specific to the use of Live Fractional Cover (LFC) status and change maps in the management of the Northern Rangelands Trust (NRT) natural resources. 
Download QGIS from: http://www.qgis.org/.

Install the 3.8 Zanzibar version or the latest available 3.10.

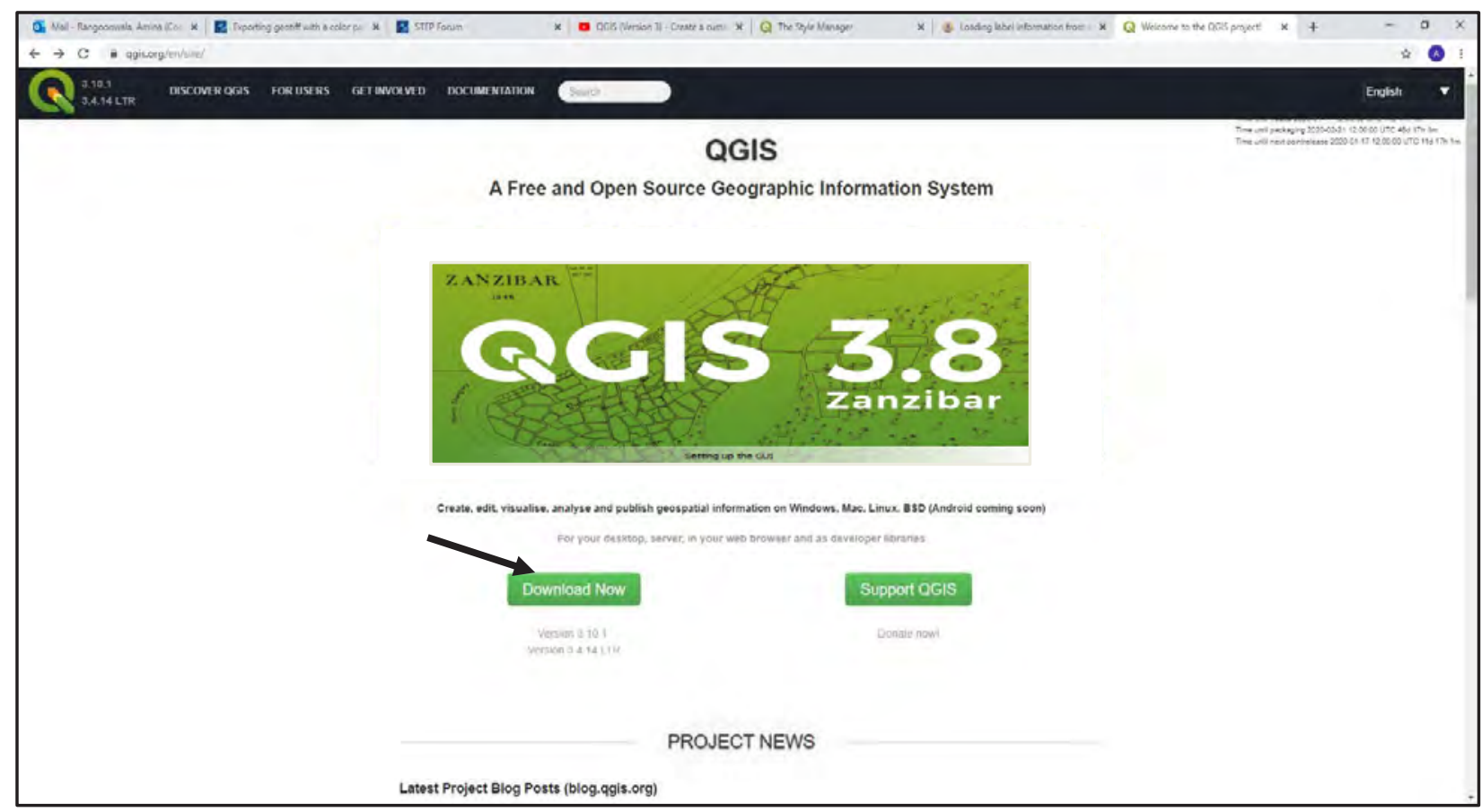




\section{Setting up QGIS.}

\section{A. Getting started.}

Set up the browser and layer: Menu $>$ View $>$ Panels. Click browser, layer, and processing toolbox.

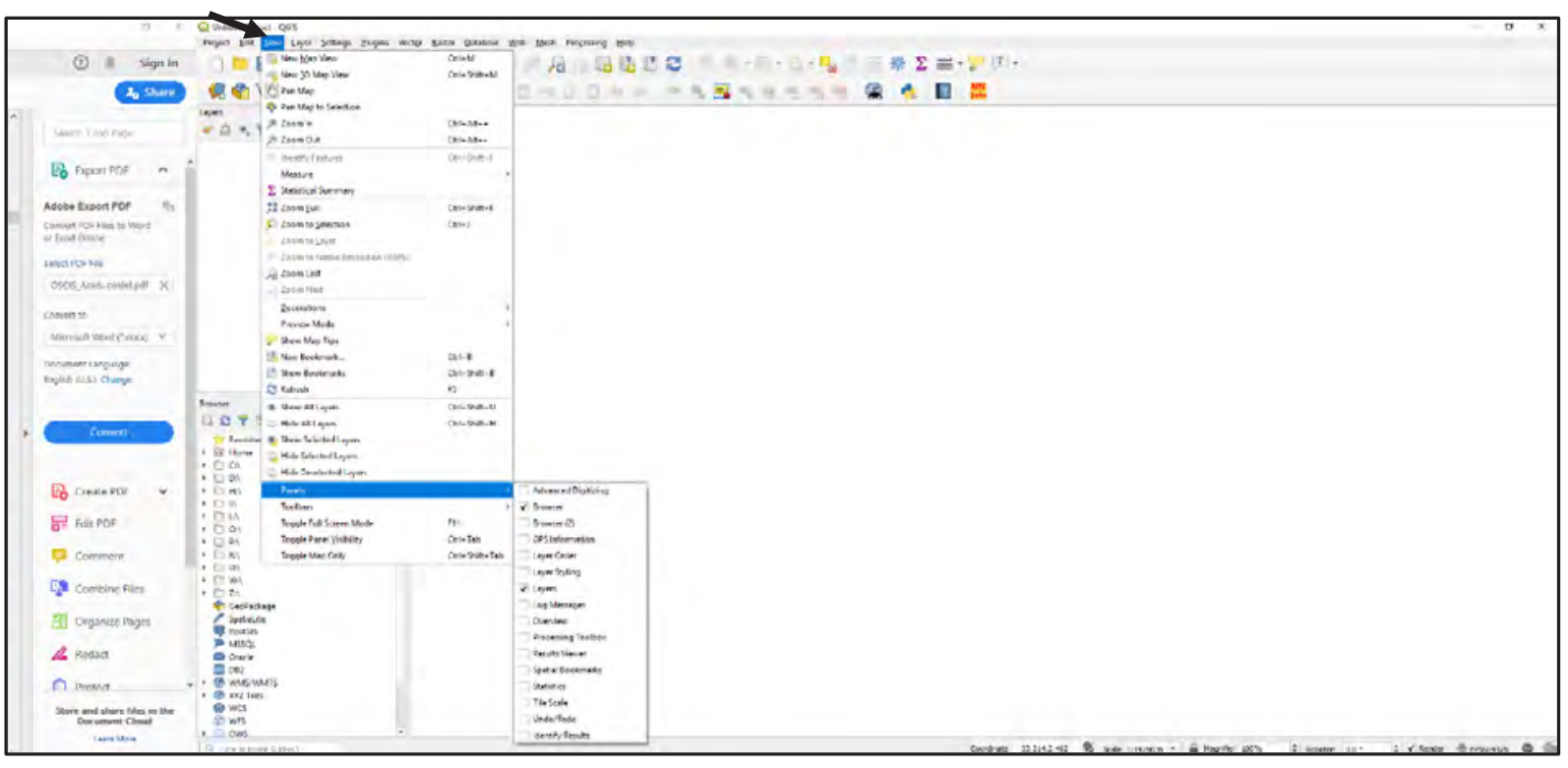

\section{B. Set language.}

Settings $>$ Options $>$ General. Set to English and do not change the default options which are already clicked.

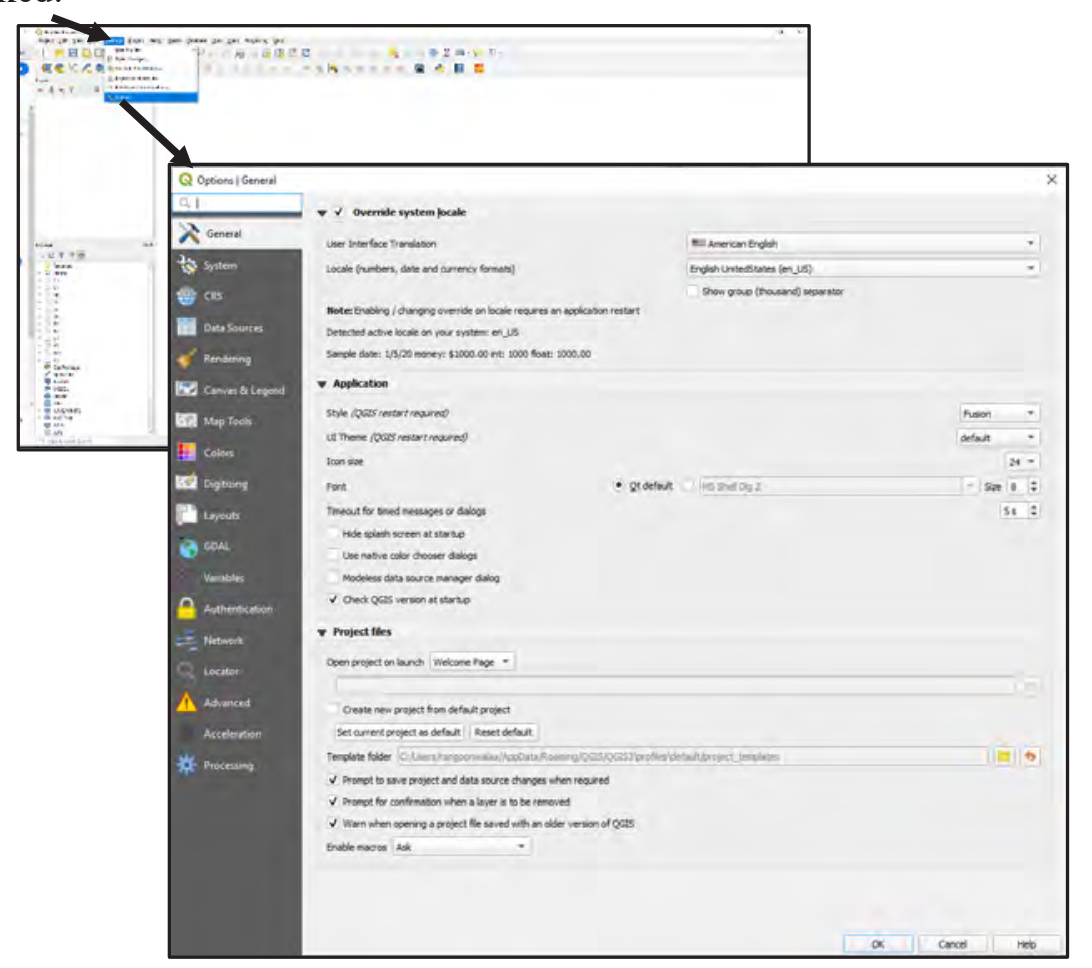

\section{C. Save project.}

Menu $>$ Project $>$ Save As. Go to the relevant folder (QGIS_workshop) and save as *.qgz. 


\section{D. Set Coordinate Reference System.}

Menu $>$ Project $>$ Properties. Select the projection for Kenya at:

http://downloads.gvsig.org/download/geodata/EPSG_codes/Projected_Coordinate_Reference_Systems.pdf.

EPSG: 32637 WGS 84 / UTM zone 37N. Filter 32637 or Select EPSG: 32637

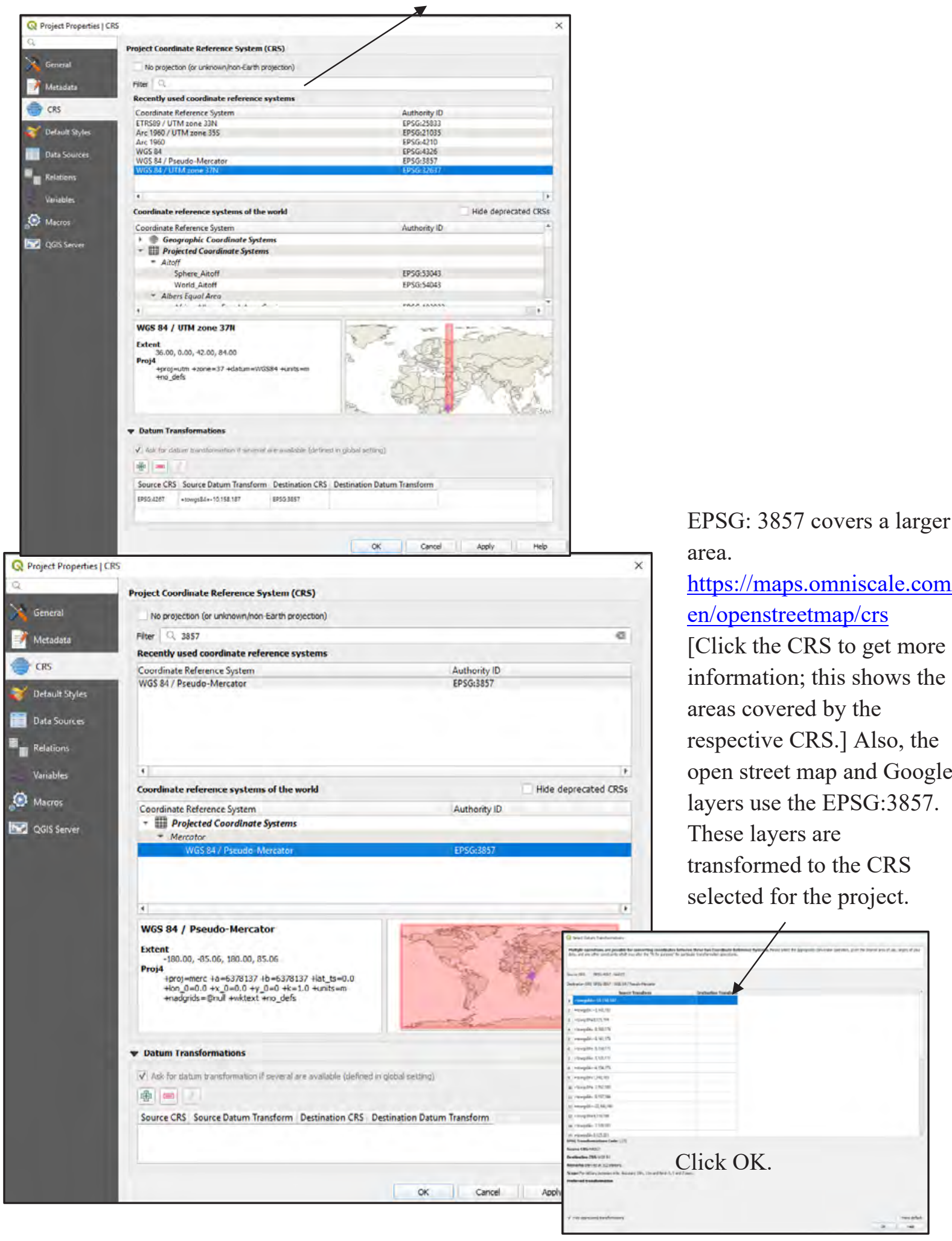




\section{Add Google Maps layers (Google Earth) in QGIS.}

Adapted from https://geogeek.xyz/how-to-add-google-maps-layers-in-qgis-3.html.

To add the Google Maps Tile Map Service (TMS) in QGIS, go to XYZ Tiles in the Browser panel. Click New Connection; right click XYZ Tiles.

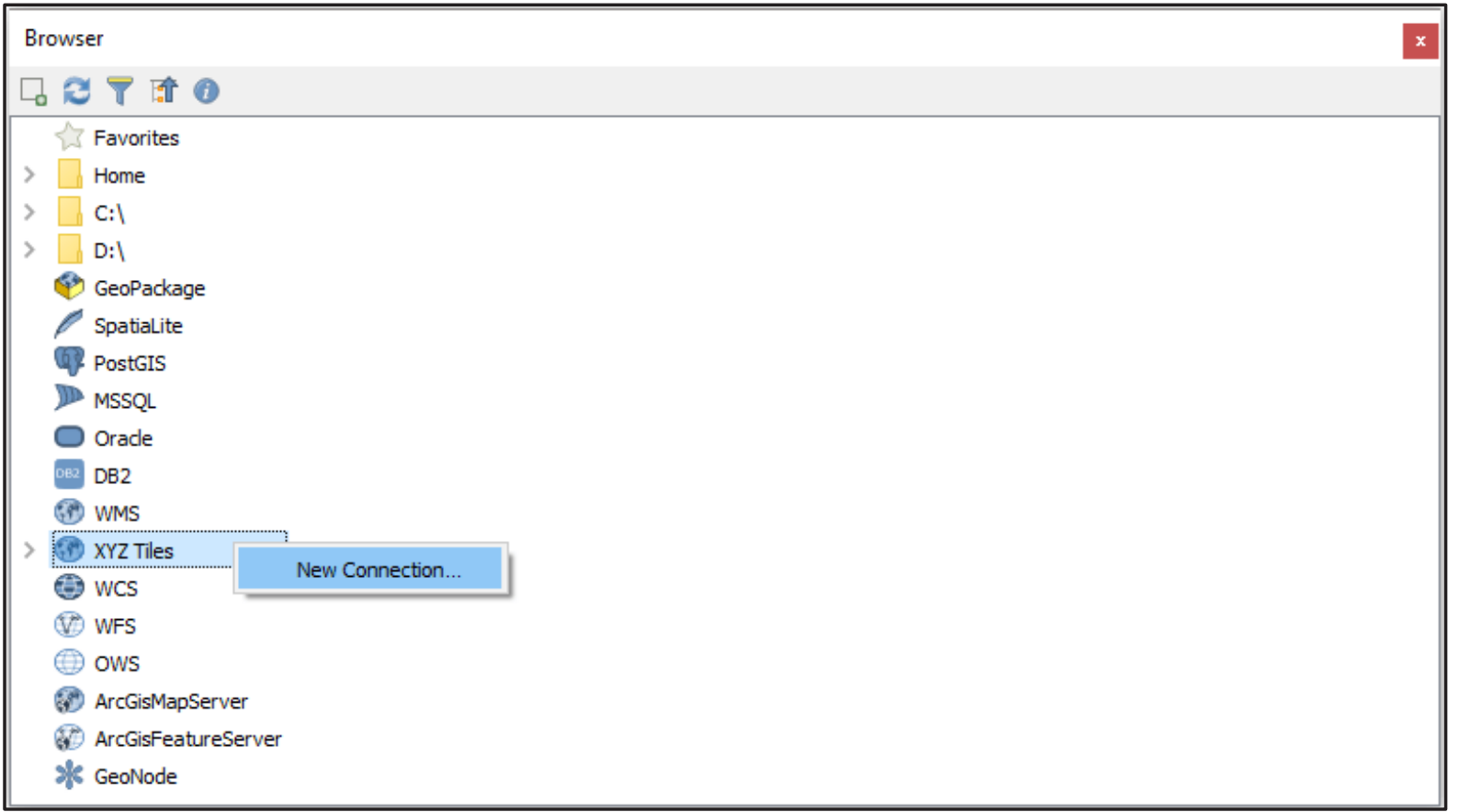

Enter the name: for example, Google Satellite. Copy and paste one of the Google Maps TMS listed on page 33 into the URL. Adjust the maximum zoom level (19). Click OK.

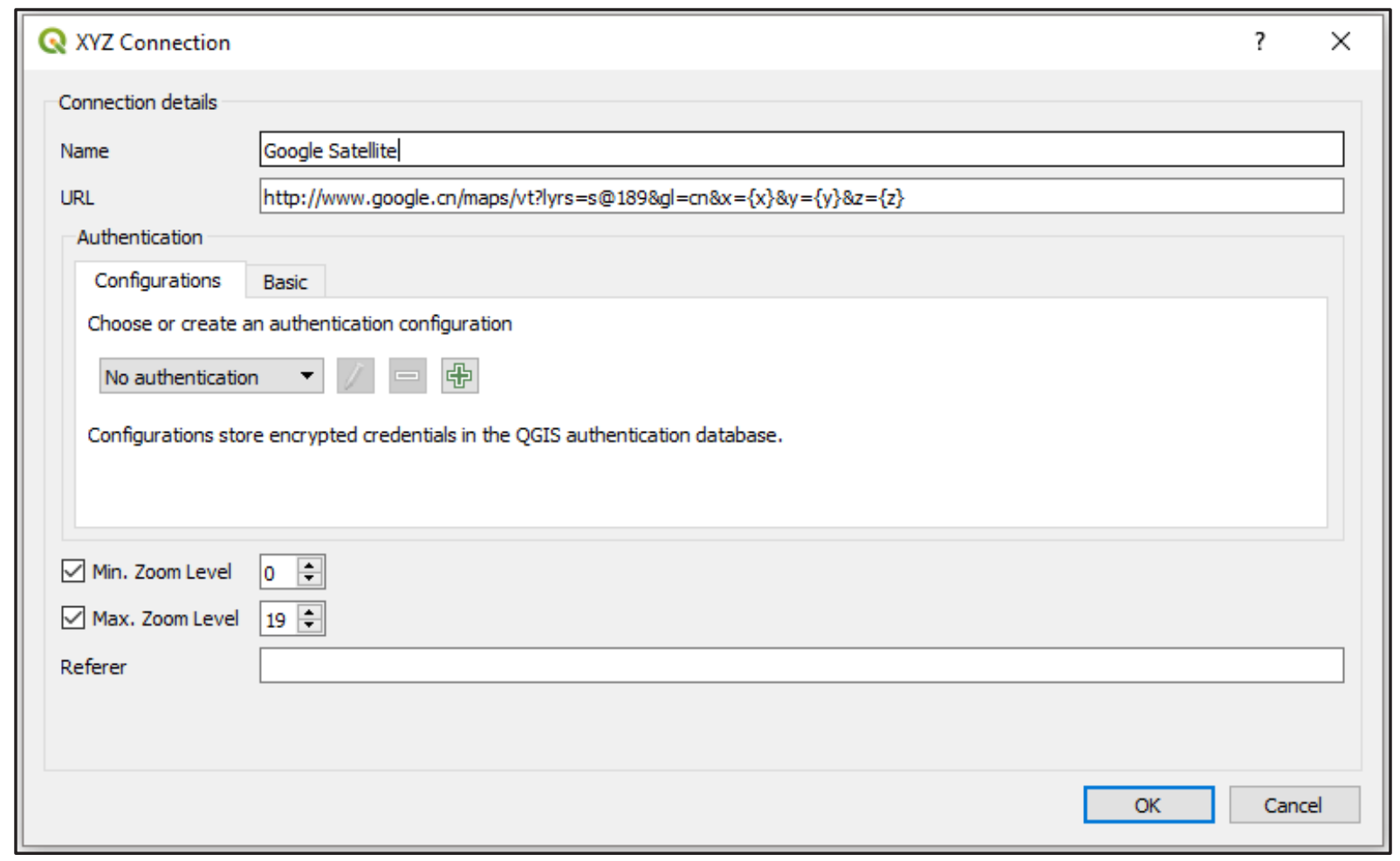




\section{Links to download the Google map layers:}

Google Maps: $\underline{\mathrm{https}} / / / \mathrm{mtl}$.google.com/vt/lyrs $=r \& x=\{x\} \& y=\{y\} \& z=\{z\}$

Google Satellite: http://www.google.cn/maps/vt?lyrs=s@189\&gl=cn\&x=\{x $\& y=\{y\} \& z=\{z\}$

Google Satellite Hybrid: https://mtl.google.com/vt/lyrs $=y \& x=\{x\} \& y=\{y\} \& z=\{z\}$

Google Terrain: https://mtl.google.com/vt/lyrs $=t \& x=\{x\} \& y=\{y\} \& z=\{z\}$

Google Roads: https://mt1.google.com/vt/lyrs $=h \& x=\{x\} \& y=\{y\} \& z=\{z\}$

One can also bring in the Google layers using python code.

\section{Link for Python scripts:}

https://raw.githubusercontent.com/klakar/QGIS_resources/master/collections/Geosup

portsystem/python/qgis basemaps.py

- $\quad$ To open the Python Console, go to Plugins Menu >>

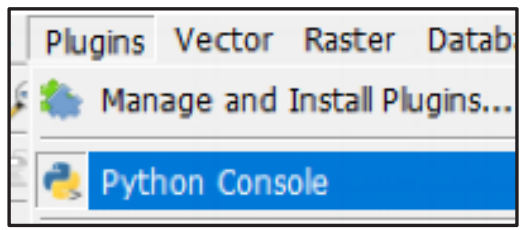

- In the Python Console window, paste in the script that you copied from the URL link above. Run it and you will see a list of base maps under the XYZ Tiles in the Browser panel.

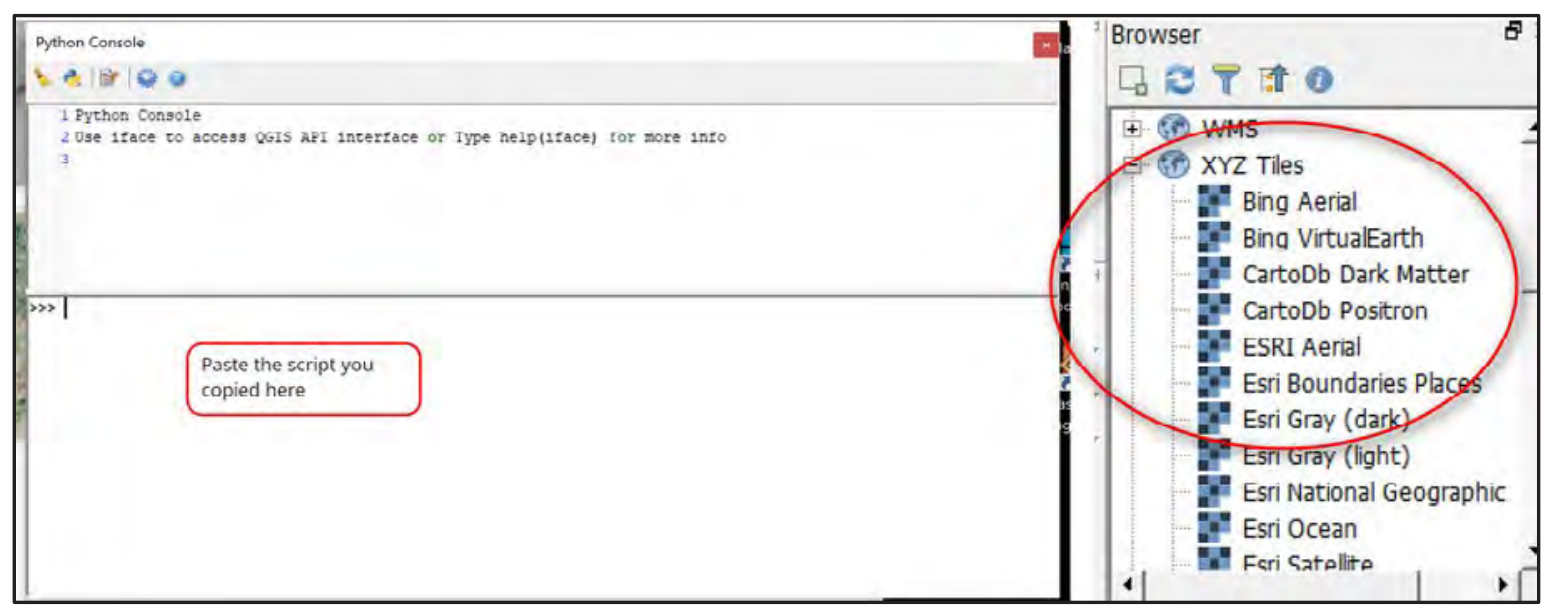

Once all layers are added to the XYZ Tiles in the Browser window, right click the applicable layer and select Add Layer to Project. Save the project.

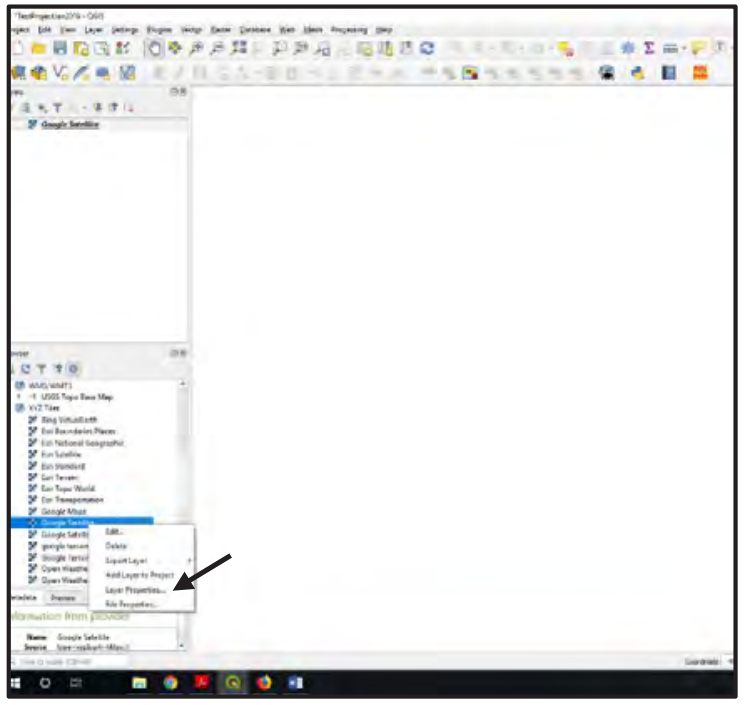




\section{A. The Open Street Map is provided as a default XYZ tile or is available in the plugin in QGIS 3 versions.}

If the OpenStreetMap raster tile is not available, follow the next steps:

Go to https://wiki.openstreetmap.org/wiki/Tile_servers.

Here, you will find a list of online raster tile servers based on OpenStreetMap data.

Select an OpenStreetMap Standard tile layer and copy the URL:

https://a.tile.openstreetmap.org $/ \$\{\mathrm{z}\} / \$\{\mathrm{x}\} / \mathbf{S}\{\mathrm{y}\}$.png.

Go to XYZ Tiles $>$ Right click $>$ New Connection $>$ Name (we will call it OpenStreetMap) $>$ URL (Paste the URL; do not forget to delete the "\$" signs when pasting the URL). Click OK.

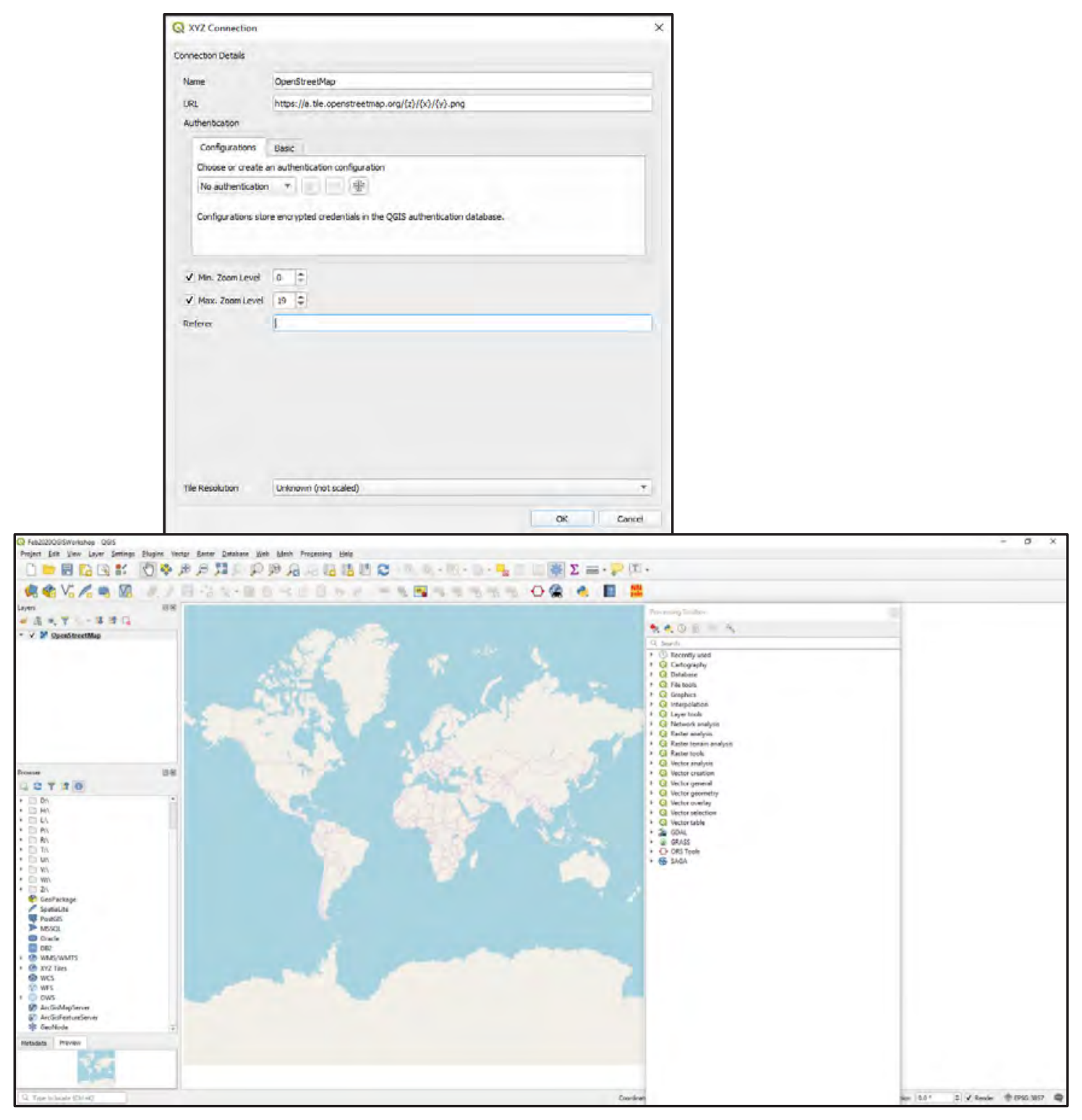

URL for open topo map:

https://tile.opentopomap.org $/\{\mathrm{z}\} /\{\mathrm{x}\} /\{\mathrm{y}\}$.png.

Add another useful plugin QuickOSM*. To add plugin follow the steps as shown in https://www.qgistutorials.com/en/docs/3/downloading_osm_data.html. 


\section{B. Add vector layer.}

For this workshop, the following folder has been created:

Data\QGIS_Training Feb2020_QGIS_WorkshopKenyalShpFiles.

Menu $>$ Layer $>$ Add Layer $>$ Add Vector Layer.

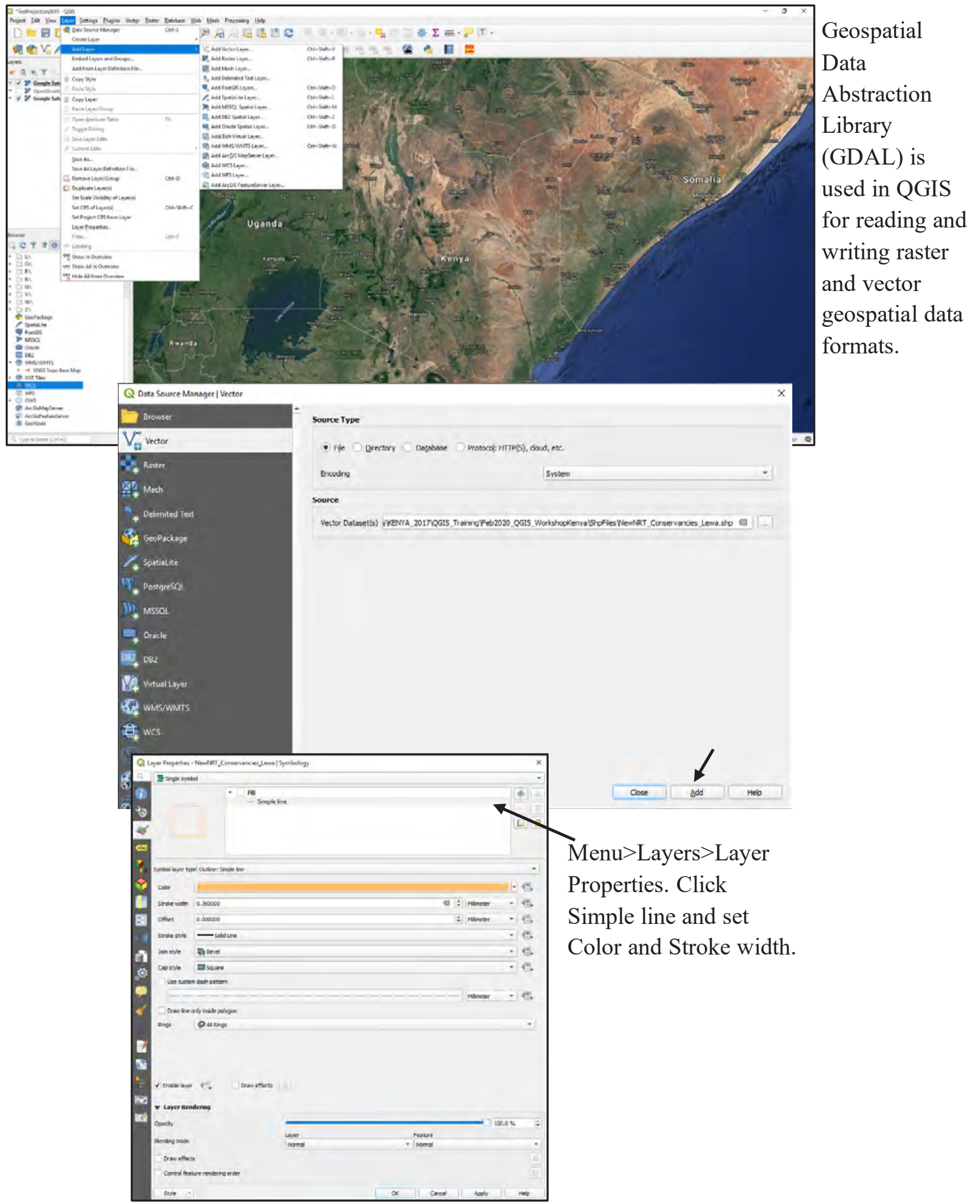




\section{C. Add raster layer.}

Browse to the relevant folder and add the 2 years of

vegetation status maps created in SNAP.

OR

Drag and drop the *.img file.

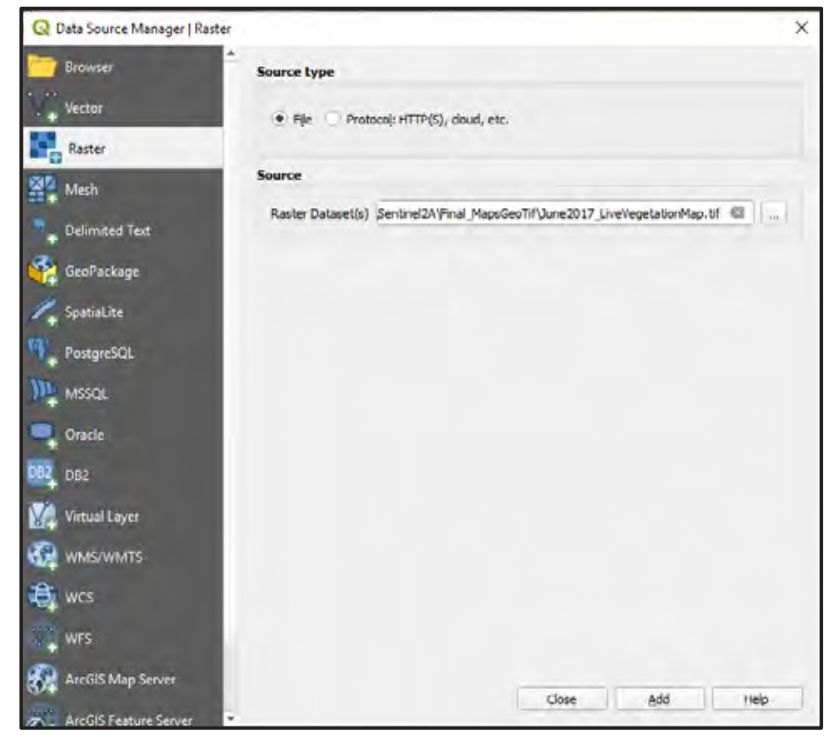




\section{Color rendering raster layer.}

Go to Menu $>$ Layers $>$ Layer Properties $>$ Symbology $>$ Band Rendering $>$ Renderer $>$ Singleband pseudocolor. Drop down on the Color ramp and select Create New Color Ramp; select Gradient, set Color 1 and Color 2 by using the drop down to choose a color. Set the RGB colors by entering the value to the right of each color $(0-255)$ or by entering the hexadecimal equivalent in the HTML notation. Repeat these steps for setting the second color (follow screen captures 1, 2, 3, and 4).
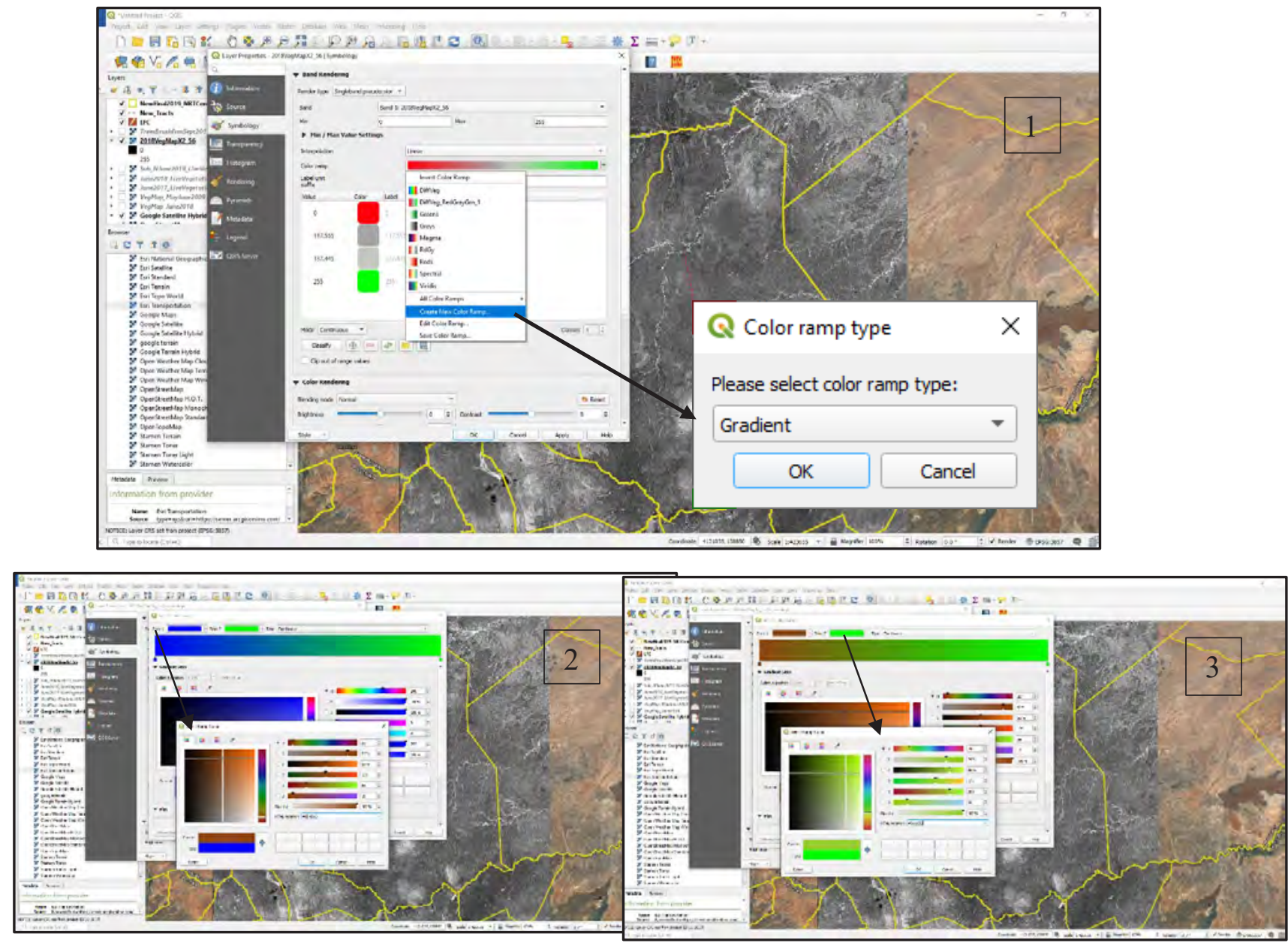

Set Color1 RGB 139, 69, 19; HTML notation \#8b4513

and Color2 RGB 154, 205, 50; HTML notation \#9acd32.

Next, save Color Ramp; see screen capture 4 on page 38 .

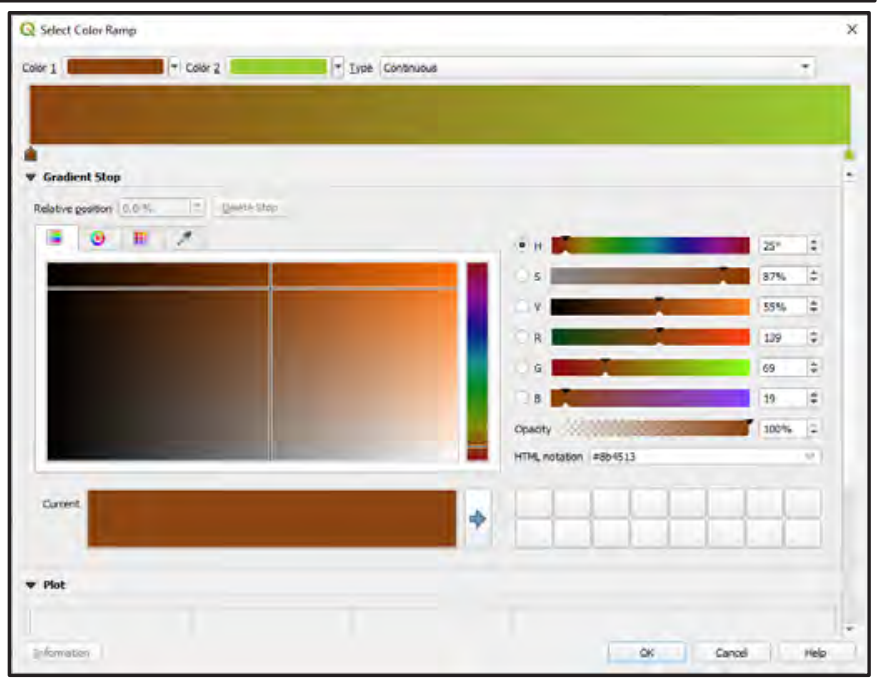


Save color ramp; Menu $>$ Layers $>$ Layer Properties $>$ Symbology.

Drop down Color Ramp; Save Color Ramp and click Apply; Type Name and Tag, click Add to Favorites and Save.

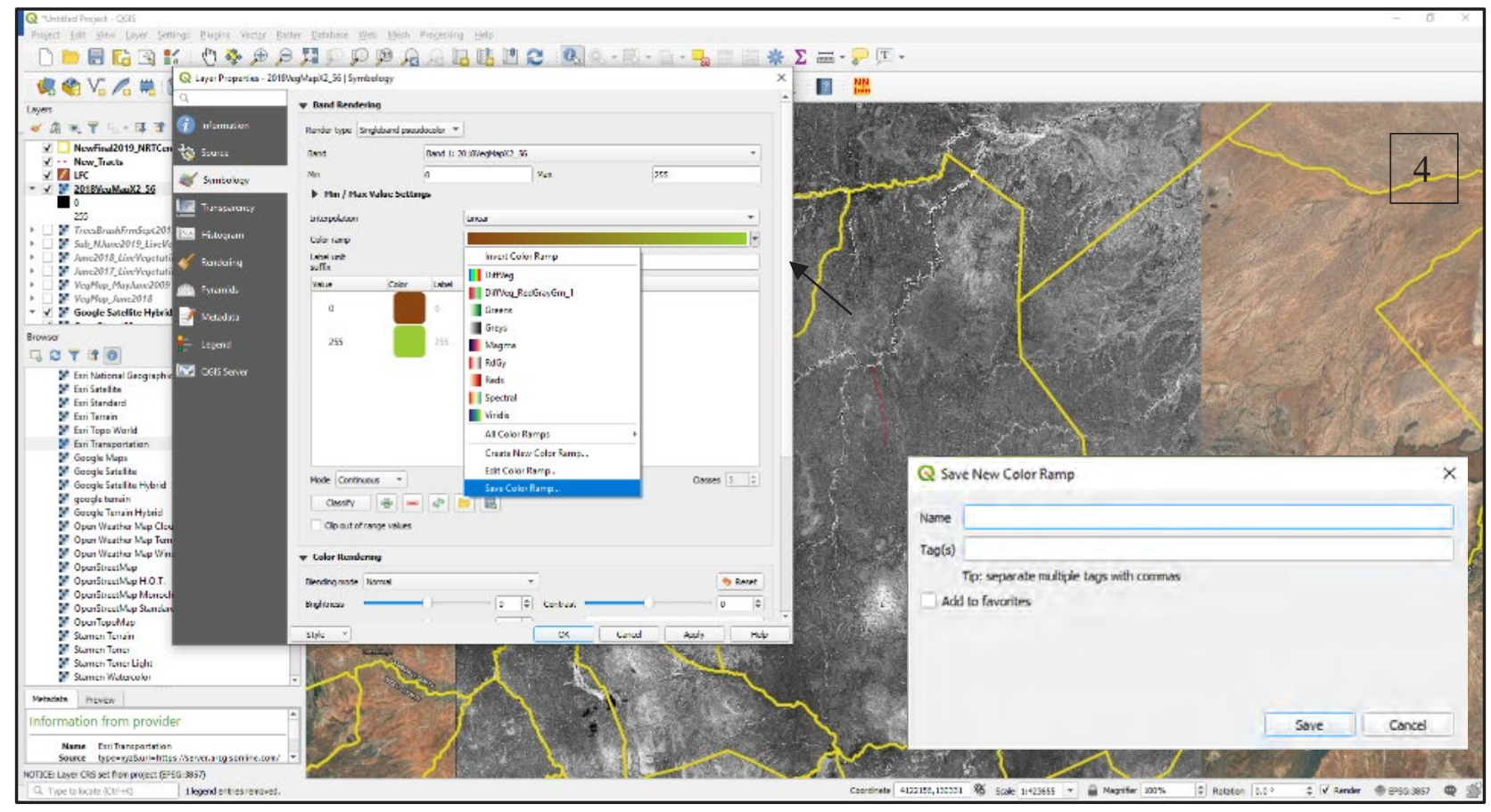

Created Color Ramp applied to the image target.

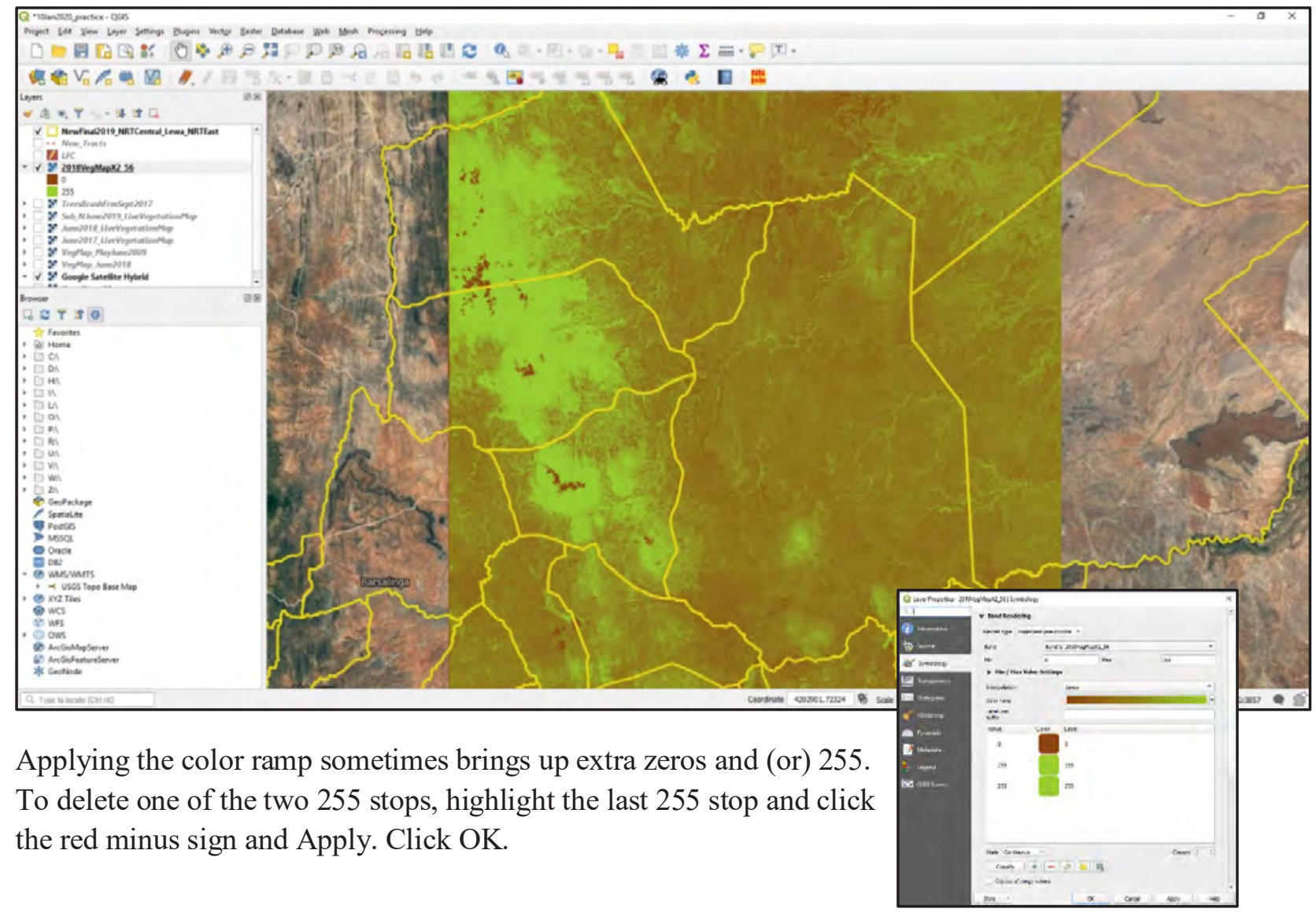


3A. Make color transparent.

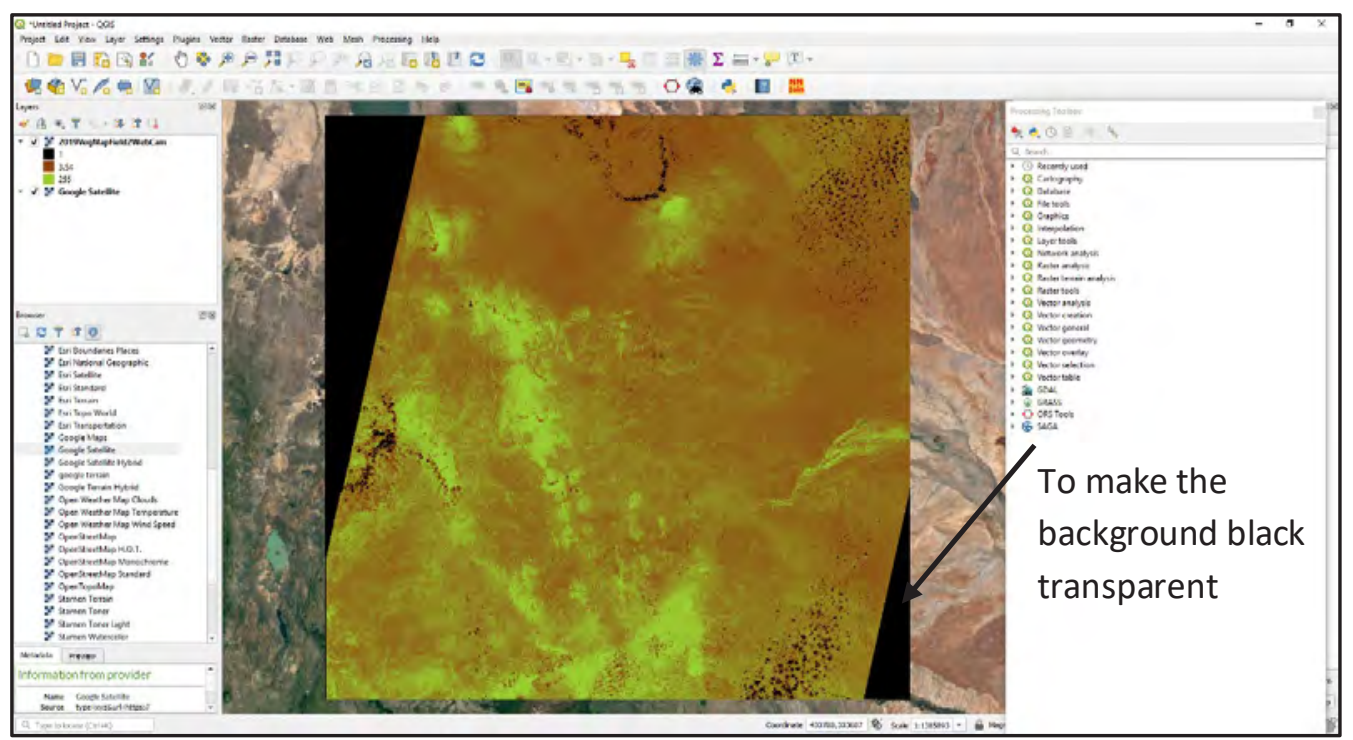

Click Layer $>$ Layer Properties $>$ Symbology. Set minimum to the SNAP minimum $>$ double click the minimum value and set opacity to zero. See screen capture below.
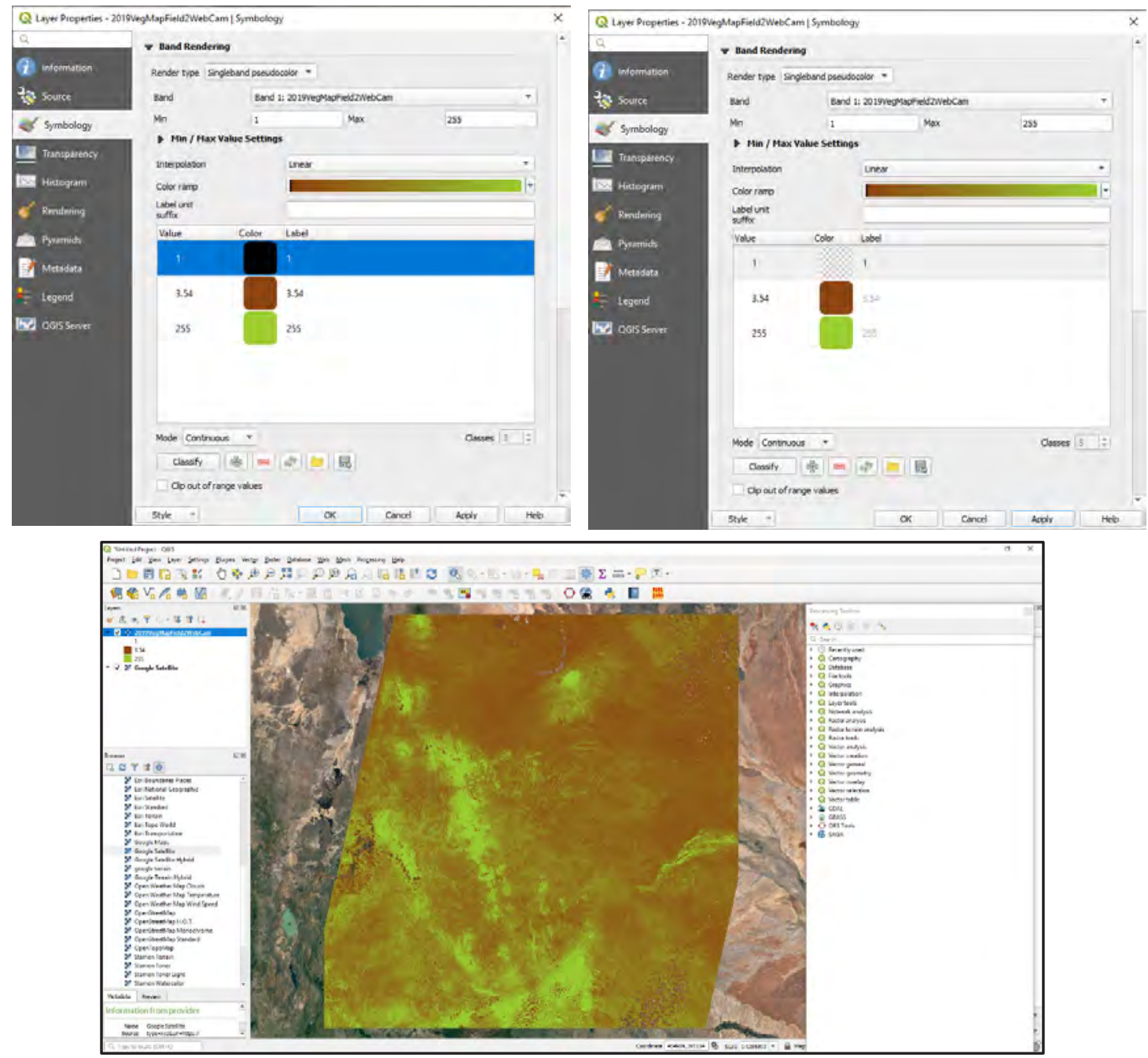


\section{B. Color rendering raster layer with gradient stops.}

Add the vegetation difference image created in SNAP(b_w16Sto256_diffVeg2019-2018): Menu $>$ Layers $>$ Layer Properties $>$ Symbology $>$ Band Rendering $>$ Render type $>$ Singleband pseudo color. Drop down on the Color ramp and select Create New Color Ramp select Gradient, set Color 1 and Color 2 by using drop down to choose a color. Color 1 RGB-0, 0 , 255; hexadecimal \# 0000ff; Color 2 RGB-255, 160, 255; hexadecimal \# ffa0ff.
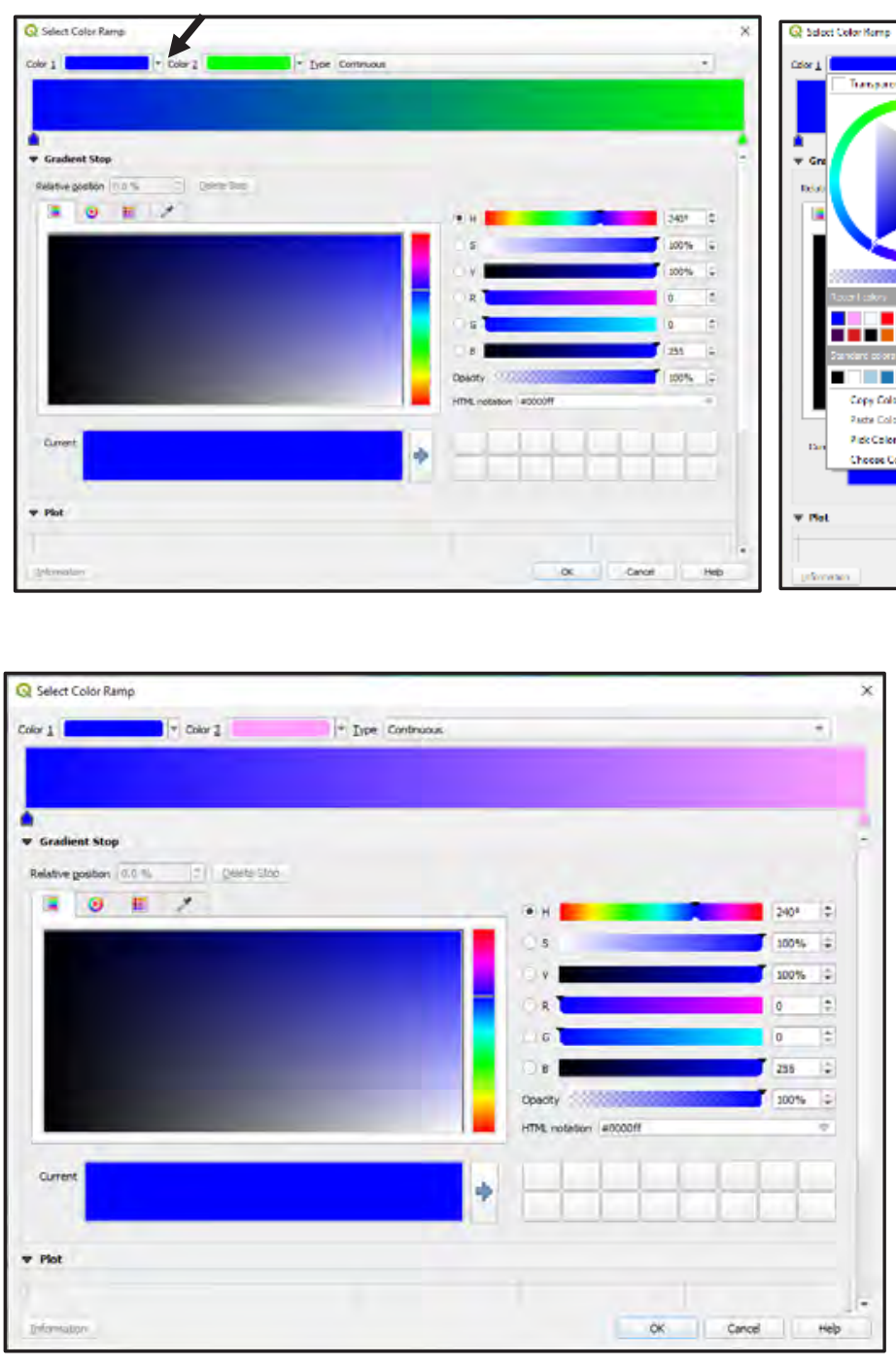

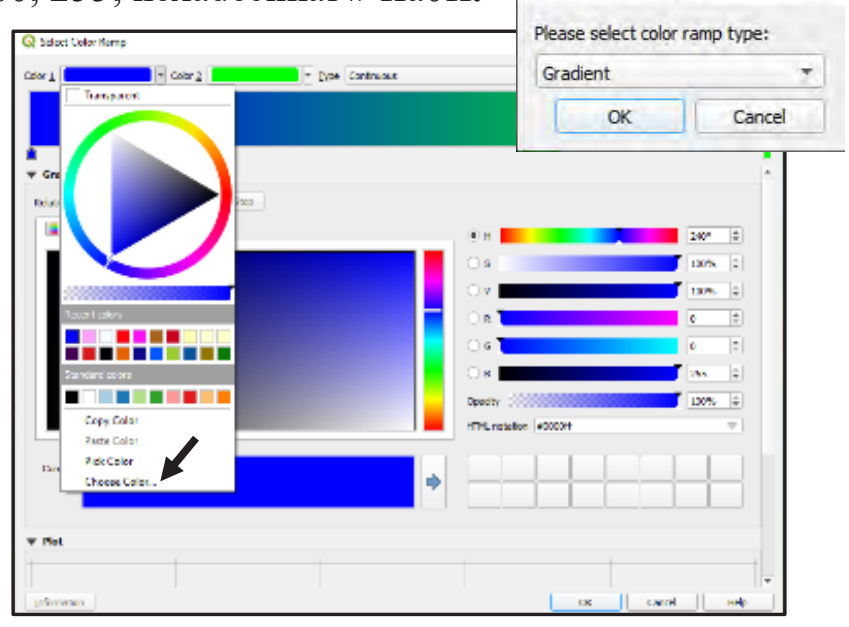

Next, set the gradient colors. Double click the top gradient color bar to add a Gradient Stop. For this pseudocolor table (PCT) we need six gradient stops plus the two endpoints, Color 1 and Color 2. Set the RGB or hexadecimal values for the color.

Set the relative position of the stop under Gradient Stop. The gradient bar only extends from 0 percent to 100 percent. Divide your 0-255 breakpoints by 256 to get the 0 percent to 100 percent estimate and use those to set the relative position. QGIS will automatically convert these to 0-255 equivalents. The relative position values are given in the table below.

\begin{tabular}{|l|c|c|c|c|c|c|c|c|}
\hline & \multicolumn{2}{|l|}{ Two-tile mosaic breakpoints } & \multicolumn{2}{l|}{ Nine-tile mosaic breakpoints } & & & \\
\hline Color & $8 \mathrm{U}-16 \mathrm{~S}-255$ & $8 \mathrm{U}-100 \%$ & $8 \mathrm{U}-16 \mathrm{~S}-255$ & $8 \mathrm{U}-100 \%$ & Red & Green & Blue & Hexadecimal \\
\hline Blue & 83.41 & 32.6 & 0 & 0 & 0 & 0 & 255 & $0000 \mathrm{ff}$ \\
\hline Cyan & 104.44 & 40.8 & 50.0083 & 19.61 & 0 & 255 & 255 & $00 \mathrm{ffff}$ \\
\hline Green & 125.89 & 49.2 & 101.0083 & 39.61 & 0 & 255 & 0 & $00 \mathrm{ff} 00$ \\
\hline Yellow & 147.34 & 57.6 & 152.0083 & 59.61 & 255 & 255 & 0 & ffff00 \\
\hline Red & 168.79 & 65.9 & 203.0083 & 79.61 & 255 & 0 & 15 & ff000f \\
\hline Pink & 189.80 & 74.1 & 252.9883 & 99.21 & 255 & 160 & 255 & ffa0ff \\
\hline
\end{tabular}




\section{C. Save the created color ramp and apply it to the black and white image.}

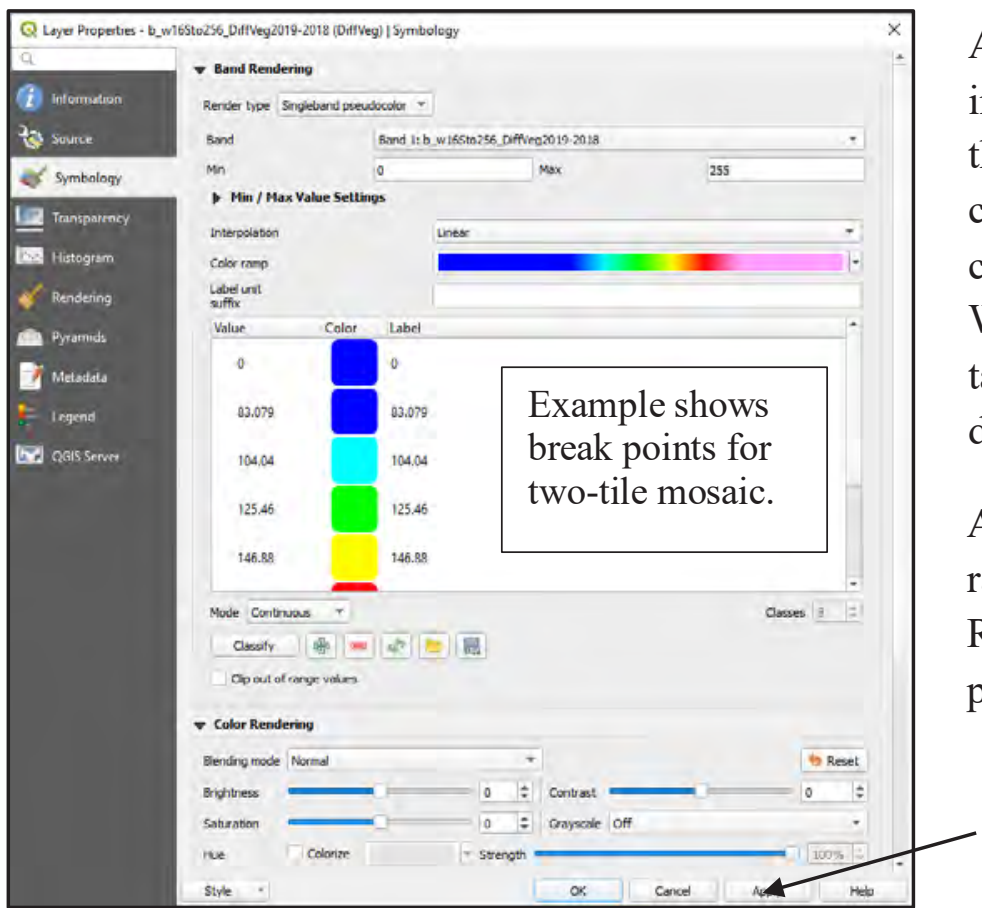

After creation of the color ramp, it is important to save it by clicking on the Color ramp drop down and clicking Save. This adds the created color ramp to the color ramp library. When saving, it will prompt for a tag, which is a descriptor used to describe and identify the color ramp.

After finalizing and saving the color ramp, make sure that the Band Rendering $>$ Render type is Singleband pseudocolor, then click Apply.

Tip: For adding or deleting Tags, Smart Groups, or a Color ramp, go to Settings and open Style Manager. In the Style Manager, right click the tag you want to delete then remove the tag. You can also add a tag in the same way. Go to All in the Style Manager to remove or edit all items.

Note: Bringing up the color ramp adds extra 0 and 255 gradient stops.

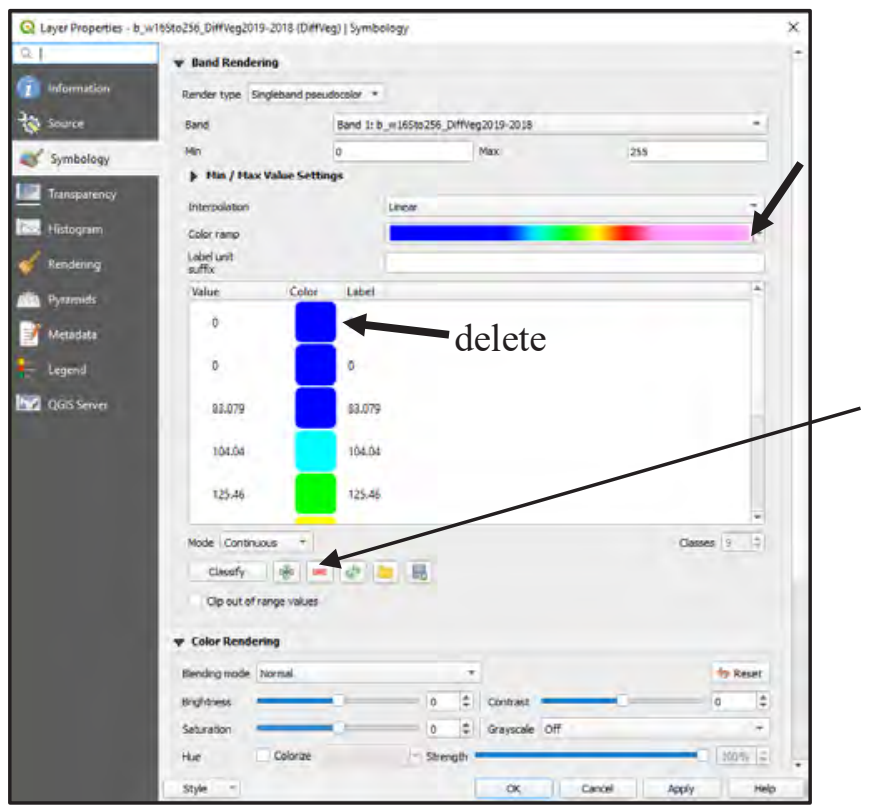

To delete one of the two zero stops, highlight the first zero stop and click the red minus sign. Click Apply, then OK. 
3D. The color ramp can also be saved as a *.txt and a *.qml.

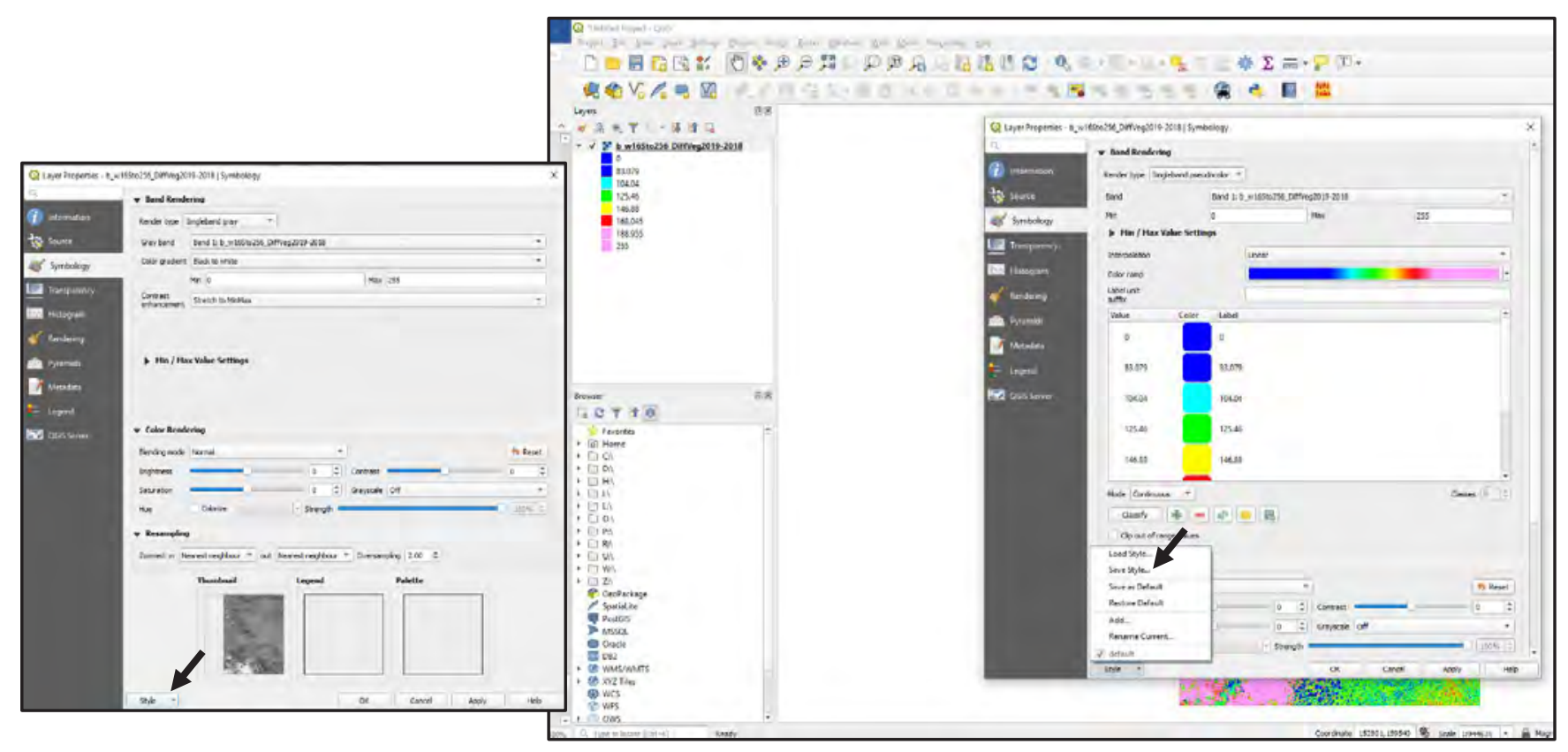

Change Render type at the top of the box to Singleband pseudocolor.

To load and attach a color ramp to a black and white, singleband image file, open the Layer Properties dialogue box. Click the Menu Layer link, then the Style button at the lower left corner of the box and Load style [*.qml]. The created color ramp can also be saved in this box as a [*.qml]. Click Apply at the bottom of the dialogue box. To save the color ramp as a text file, click the disc icon at the bottom of the Min/Max Value Settings on the same dialogue box. To load a text color ramp, click the folder icon located left of the disc icon. 


\section{Create a New Shape File.}

Menu $>$ Layer $>$ Create layer $>$ New Shape File Layer.

Filename: Select the browser where the file will be saved, and type in a name (for example, New Tracts).

File coding: UTF-8 (UTF-8 requires $8,16,24$, or 32 bits [one to four octets (bytes)] to encode a Unicode character; it is commonly used).

Geometry type: Drop down and select line; there are other options such as point, polygon, and so forth.

Select coordinate system.

New Field options: Status.

Type: Select text data. Click Add to Fields List.

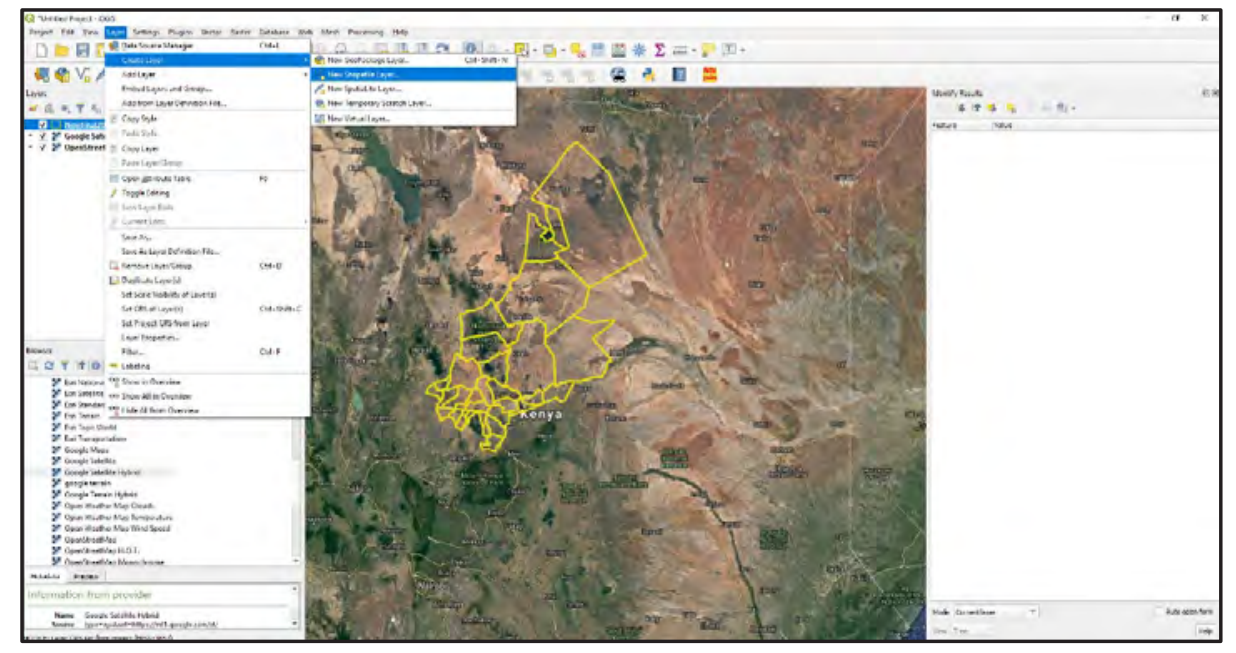

Next, create another *.shp file with File name LFC; File encoding UTF-8;

Geometry type as polygon; attributes identifier (id) as LFC, BioWt, and VegType. Click Add to Field List as you create each id.

We created two empty datasets, one a line *.shp file and the other a polygon *.shp file.

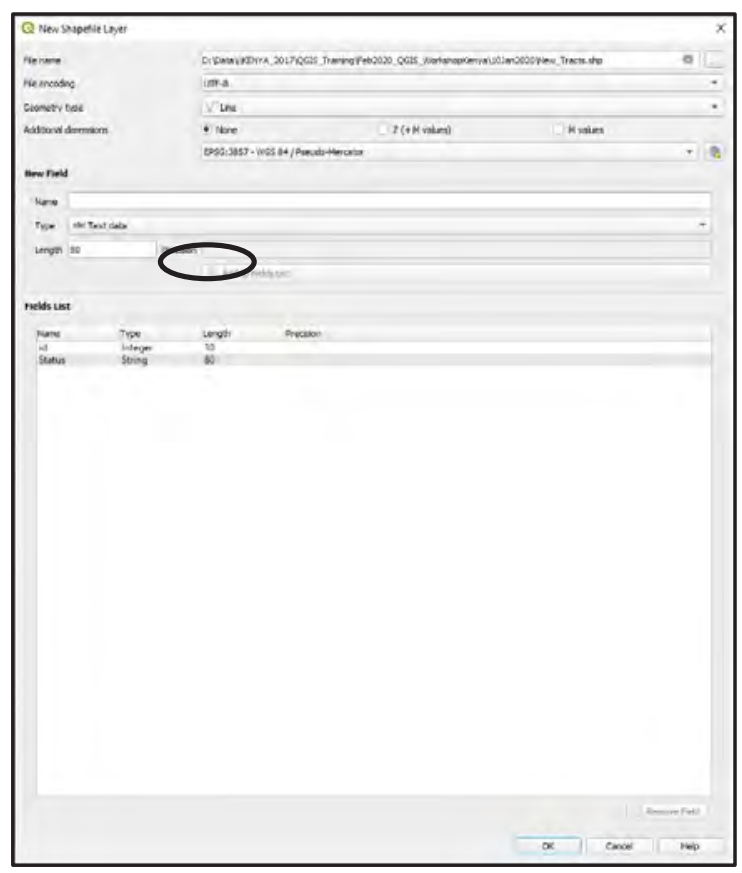




\section{A. Populating the empty line shape file.}

Select the empty shape file layer New Tracts created in step 4. Create a New Shape File. Click the pencil icon and vector editing tool. A digitizing tool appears. Zoom into the area of interest and start digitizing. When complete, right click to save the attribute information. In the dialogue box that appears, type in the identification (id) and status then click OK (screen capture 2).
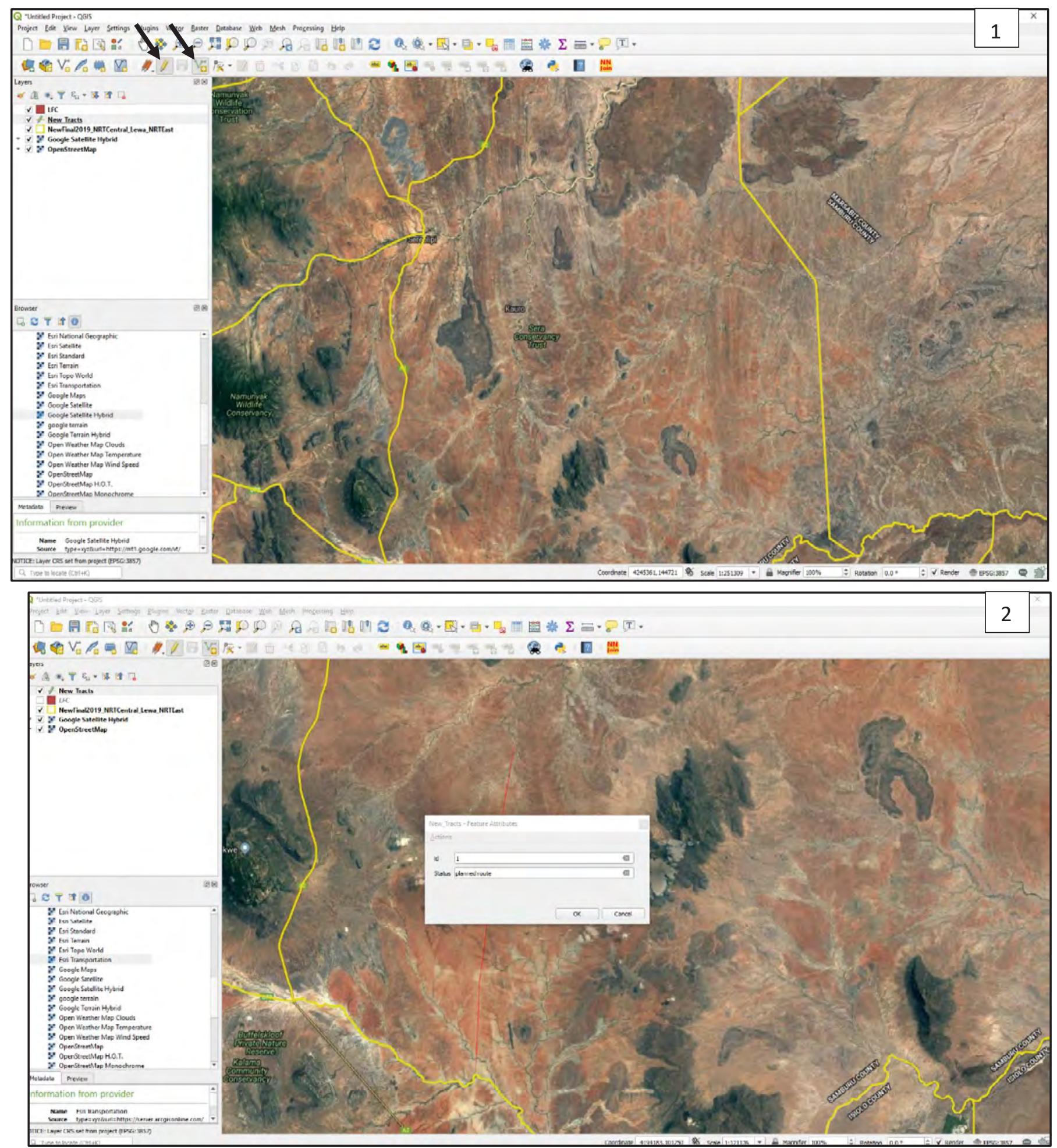


\section{B. Populating the empty polygon shape file.}

Select the LFC polygon shape file layer created in step 4. Create a New Shape File. Click the pencil icon and the polygon editing tool. A digitizing tool appears. Zoom into the area of interest and start digitizing. When complete, right click to save the attribute information. In the dialogue box, type identification (id) and status; click OK (as shown in screen capture 2 on page 44).
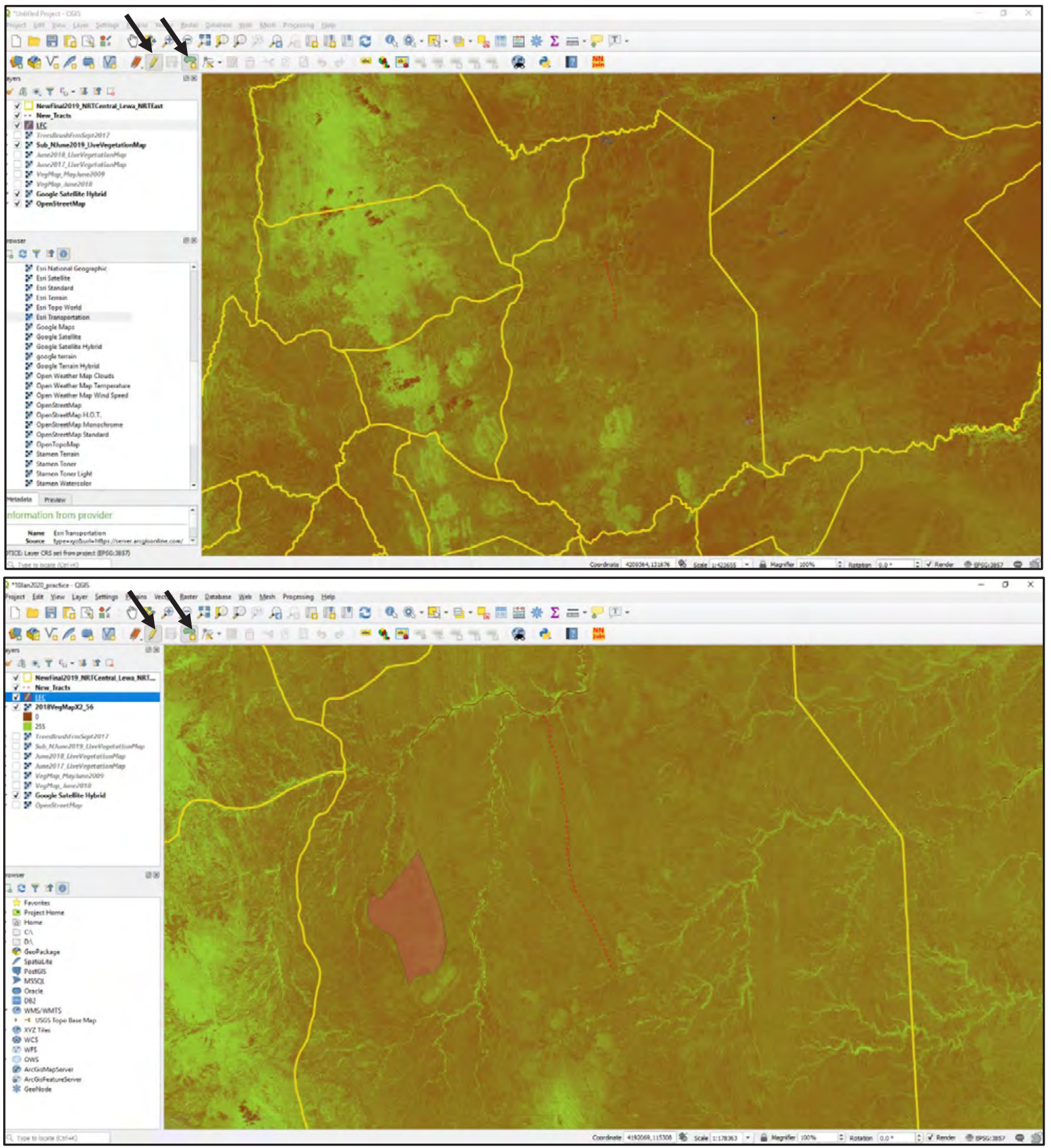


\section{C. Edit or delete attributes saved.}

Always click the edit toggle icon (yellow pencil) then right click on the *.shp layer open attribute table; select the layer you want to delete and drop the selected layer in the trash bin. To edit numbers or text, click the item and type the new numbers or text.
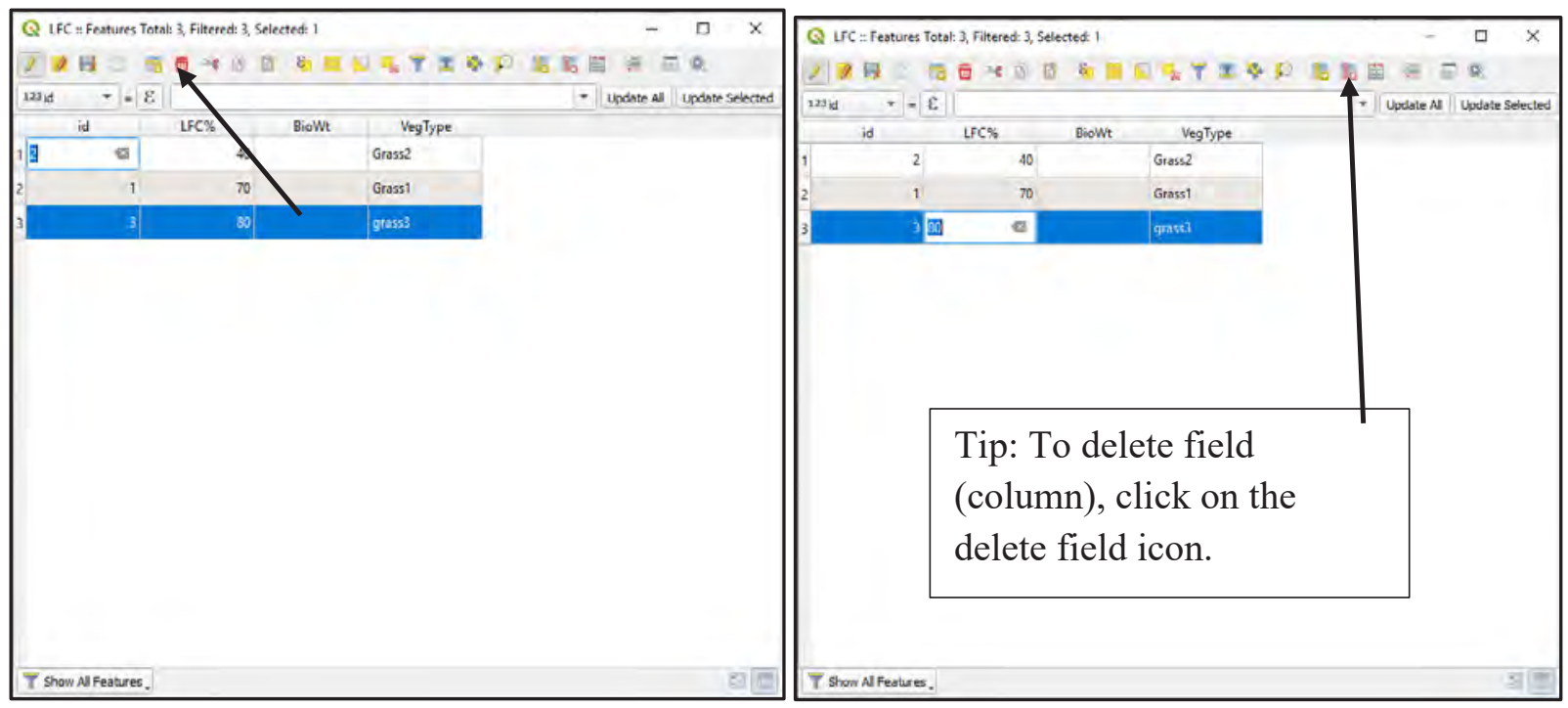

The attributes have been made arbitrarily. LFC percent cover attribute was determined from the map. Take an approximate value of the LFC cover using the identity icon. Note that the values are from 0 to 255 . To obtain $0-100$ percent, divide the identity value $\mathrm{X} / 2.55$.

\section{D. Creating a point shape file.}

Create another *.shp file: Menu $>$ Layer $>$ Create Layer $>$ New Shape File Layer.

File name: Livestock; Geometry type point; attributes identifier (id) as Owner, Cattle, Goats, and so forth, and Count; Add each to Field List that was done when creating the vector line and polygon *.shp files. Right click the layer. Open attribute table; the screen capture below shows the attribute table of the point shape file created.

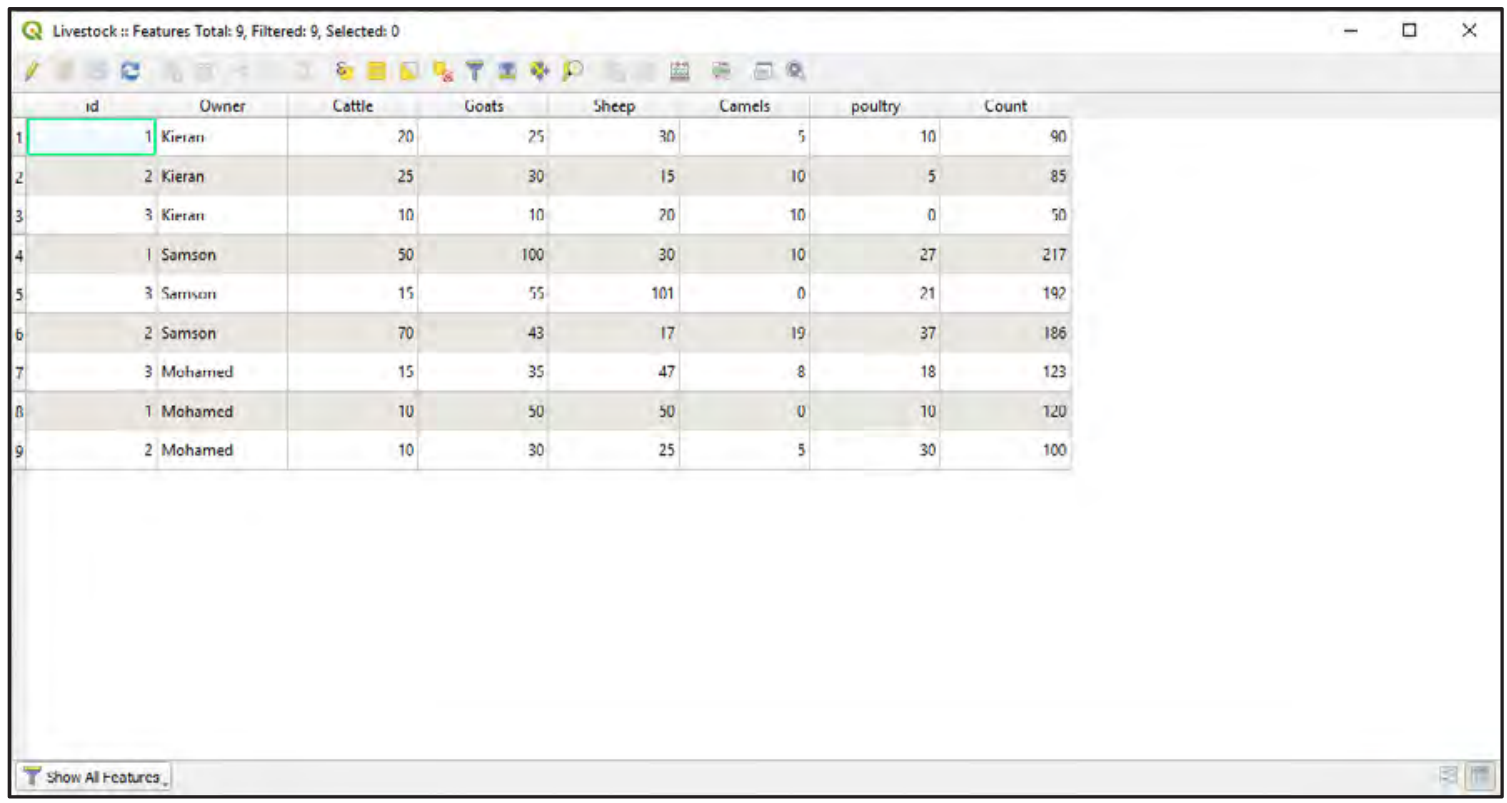




\section{Change Attribute Type.}

https://wiki.tuflow.com/index.php?title=QGIS_Change_Attribute_Type

Review the current field and data types in your table by right clicking on the layer in your layers panel and selecting Properties.

Click the fields panel on the left-hand side of the Layer Properties window. The default pBlockage data type generated by TUFLOW is type 'double.'

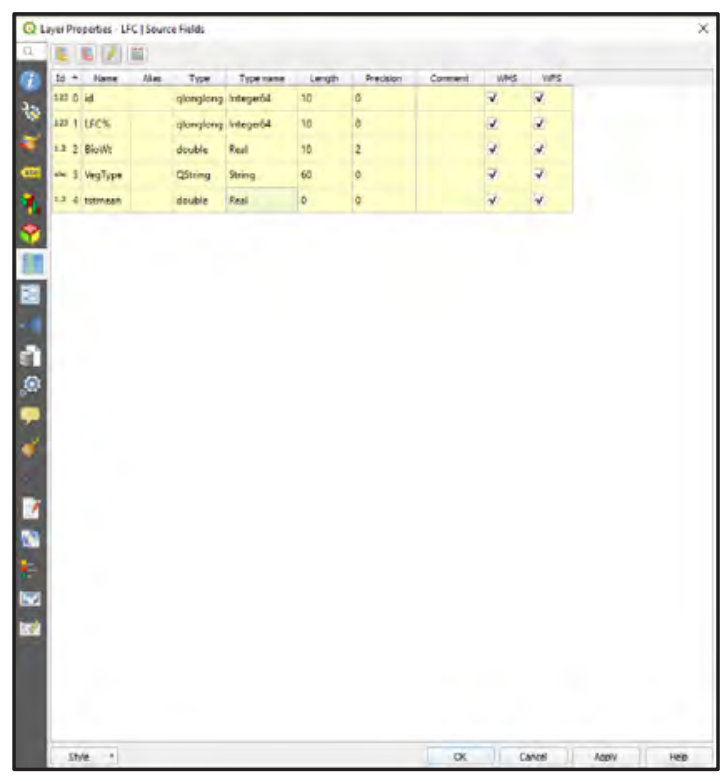

To change the datatype, use the refactor field tool located within the QGIS processing toolbox. To find the toolbox, click the Processing dropdown menu and select toolbox, as shown below.

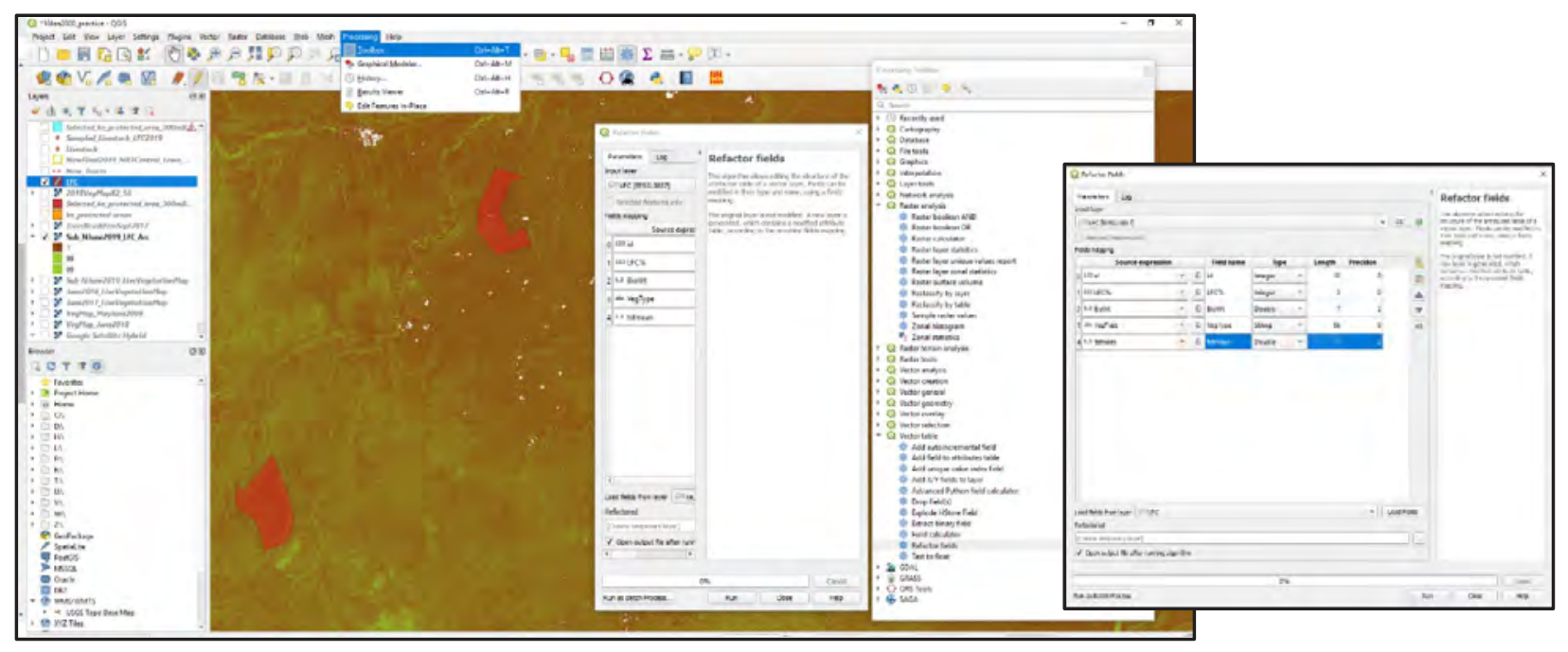

In the Processing toolbox, click Vector Table $>$ Refactor Fields. On the Refactor Fields dialog box, set the formats of the field variables, as shown on the screen capture of the Refactor Fields dialog box. Click Run; the fields will be formatted according to the format entered. The format will not be applied to new fields added to the tables after the Refactor Fields run. 


\section{Working with shape files.}

\section{A. How to formulate query expressions.}

Select the applicable *.shp file. Open attribute table; click the Expression icon $(\mathcal{E})$ shown by the red circle on screen capture (below, right). In the Expression dialog box shown on the left, expand Field and Values. Double click Owner. Next, click Operators in the same panel and click $<>$. In the next panel, select All Unique and then select Kieran. In the attribute table to the right, all owners not Kieran are highlighted. For string variables use "Owner" $<$ 'Kieran' (note: single and double quotes). Similarly, two more expressions are shown in screen captures 2 and 3. Follow instructions shown on 2 and 3.

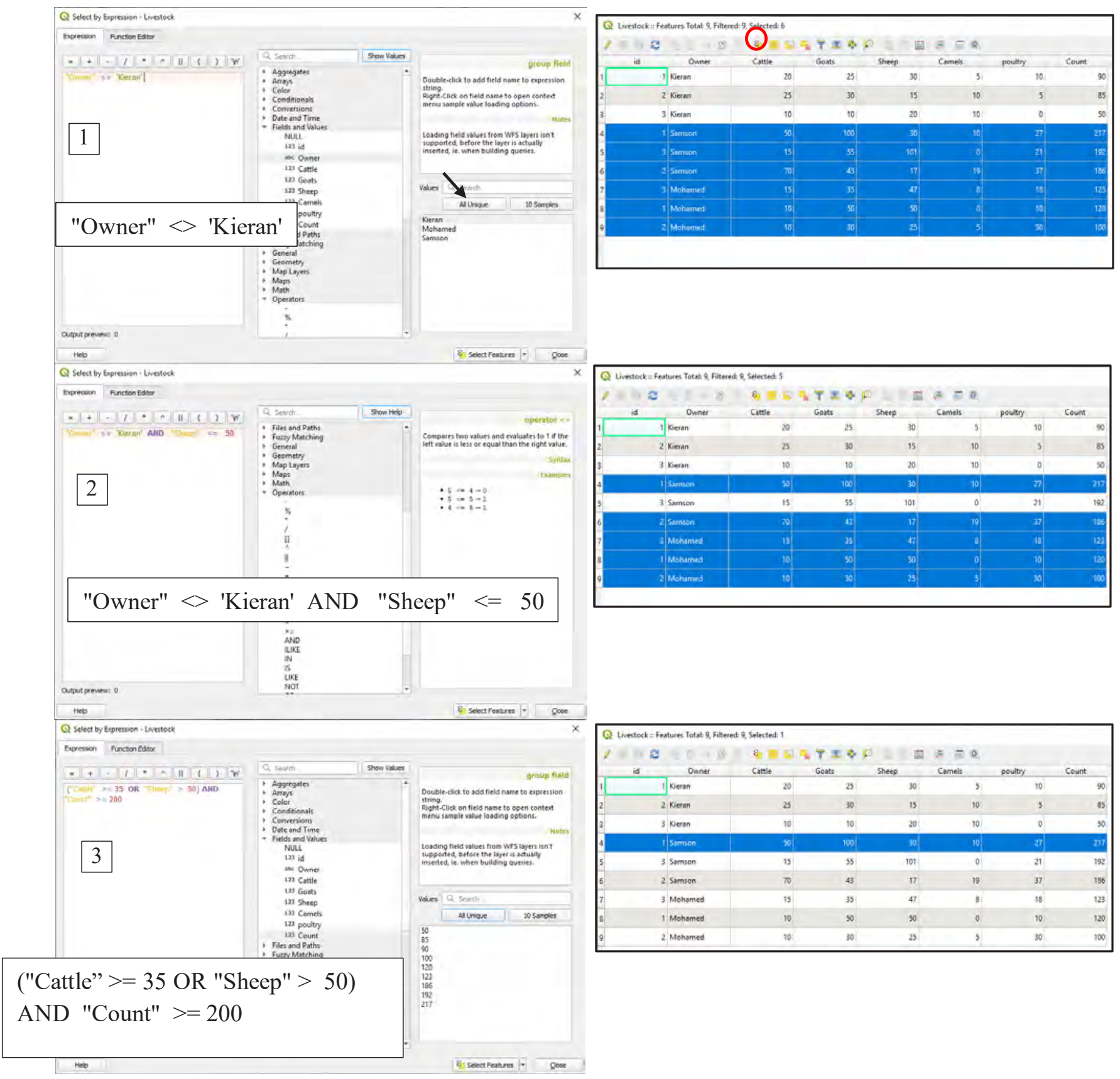




\section{B. Sample raster values with selected points.}

Objective: Retrieve LFC at three livestock points.

On the QGIS menu, click the Feature Selector icon (located by arrow). Select single or multiple points. Once livestock points are selected (shown in screen capture 1), go to Menu $>$ Processing $>$ Toolbox $>$ Processing Toolbox $>$ Raster Analysis $>$ Sample Raster Values. A new layer (Sampled Livestock LFC 2019) is created in QGIS Contents Panel. Right click the Sampled Livestock LFC 2019 layer and open the Sampled Livestock LFC 2019 Attribute Table shown in screen capture 2.
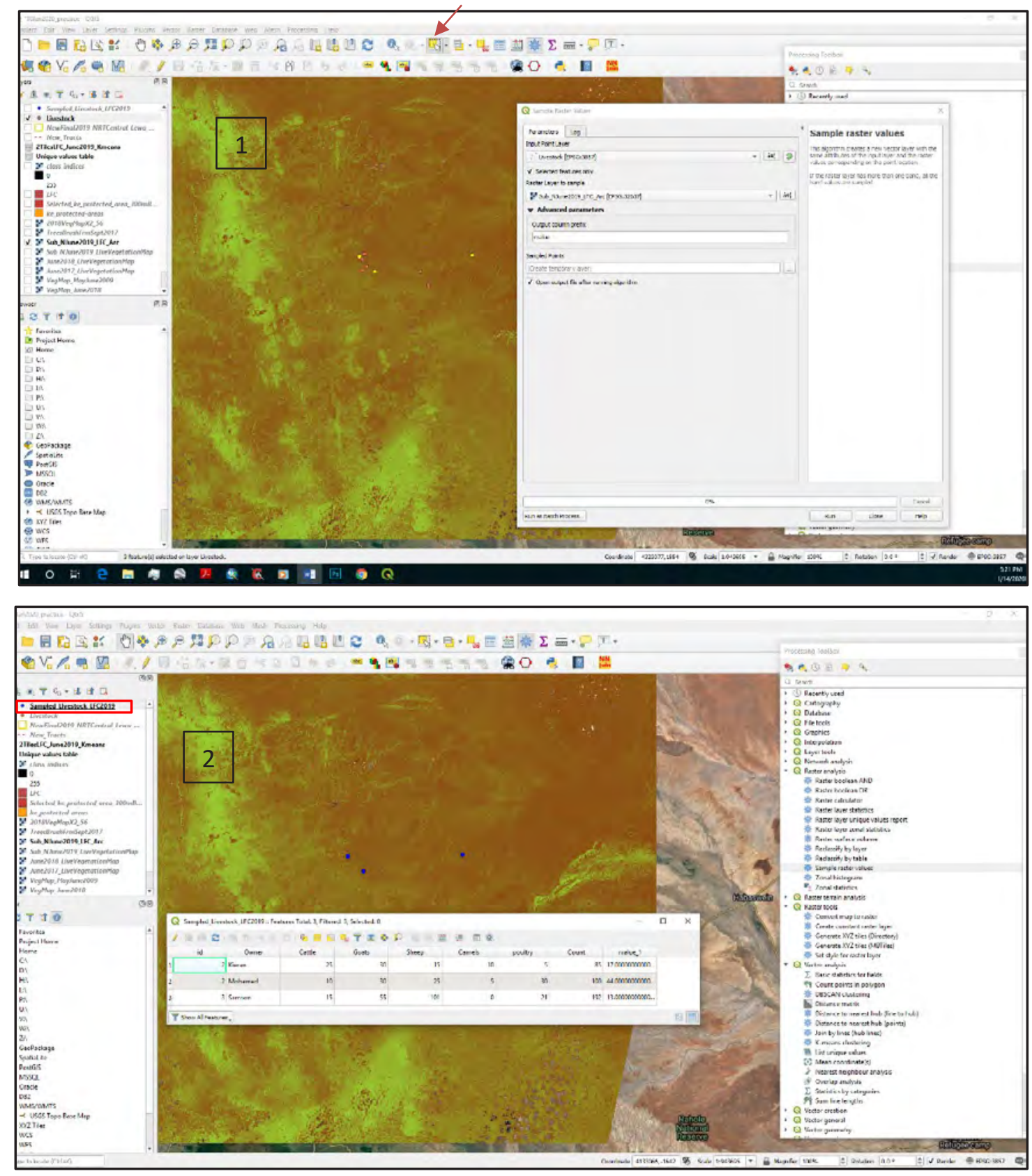


\section{Clip raster, raster calculation classification.}

\section{A. Clip raster covering the area of interest for queries.}

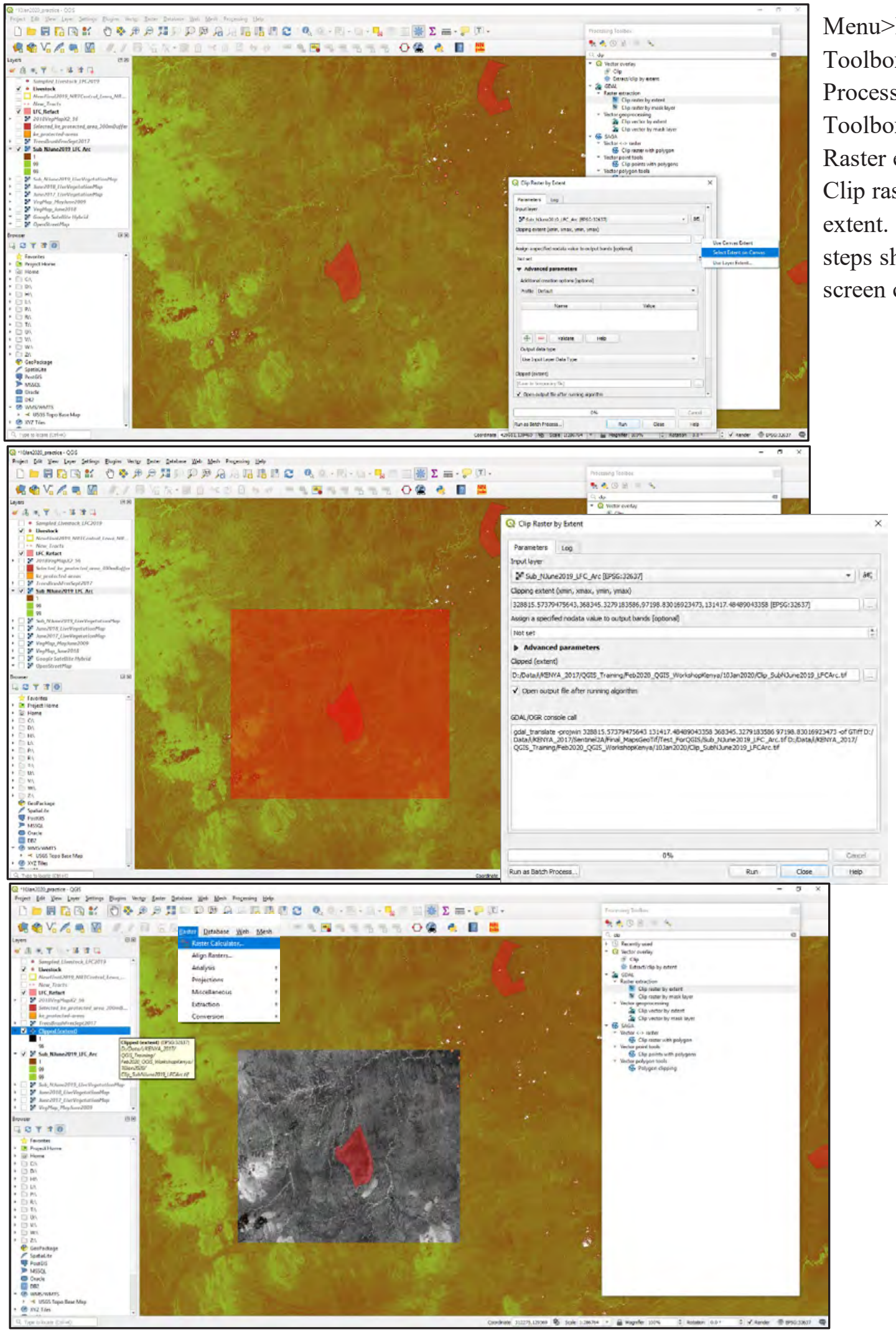




\section{B. Convert map range.}

To convert 0-255 from SNAP to $0-100$ for direct interpretation of live vegetation fractional cover status and change.

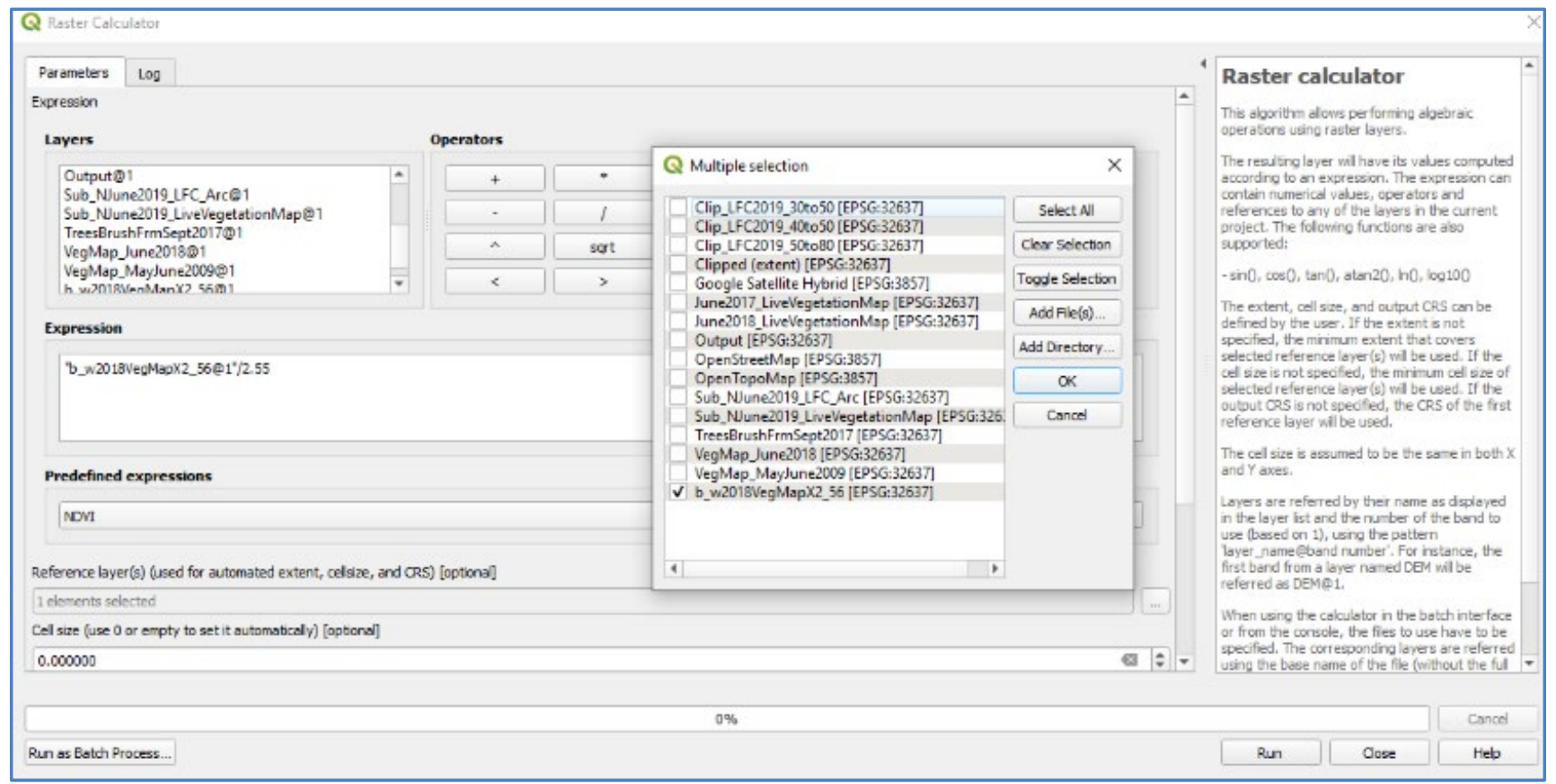




\section{C. Raster calculator, selecting pixels with LFC coverage with user defined range.}

Processing tools $>$ Raster analysis $>$ Raster calculator.
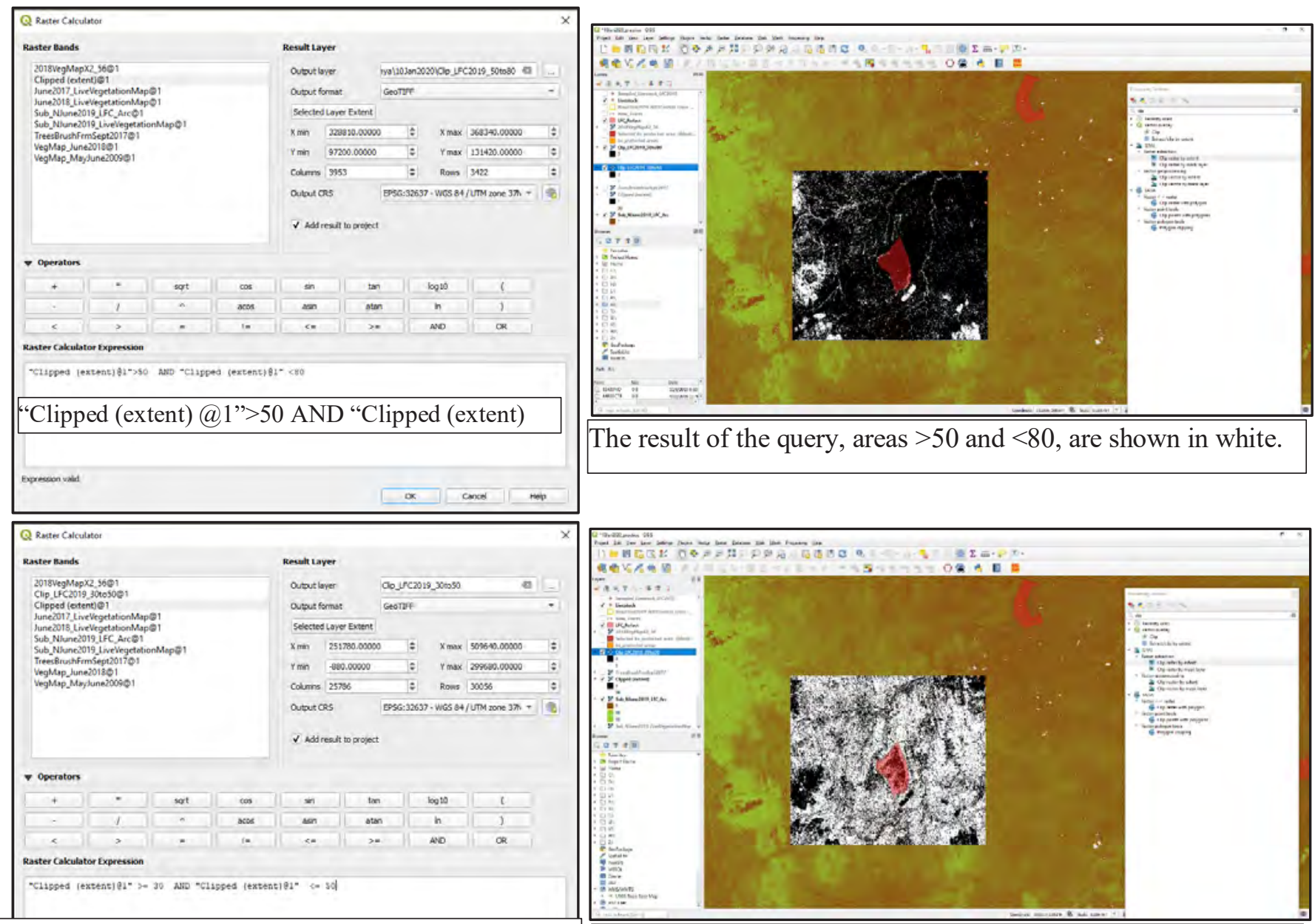

The result of the query, areas $>50$ and $<80$, are shown in white.

“Clipped (extent)@1”>=30 AND “Clipped (extent)@1"
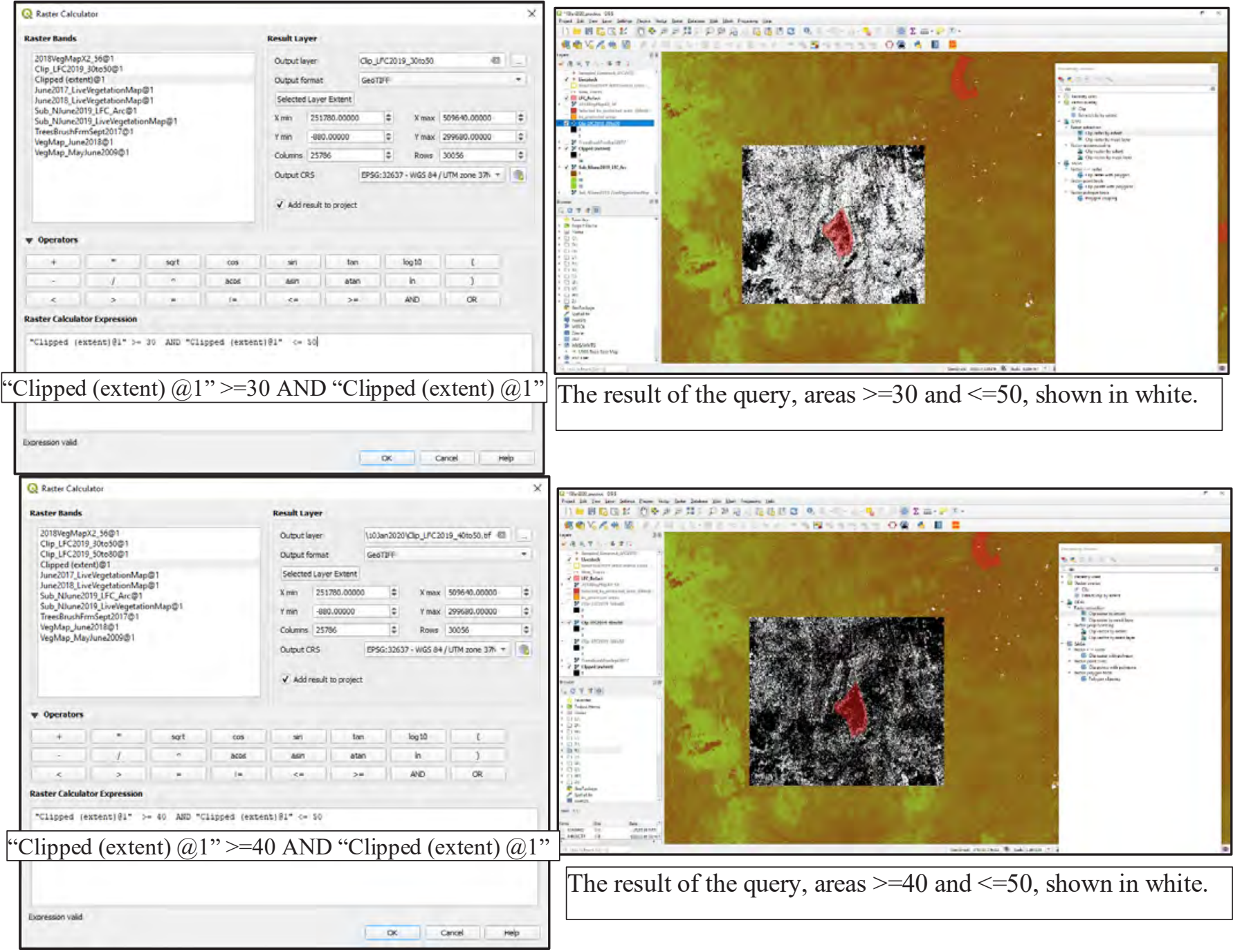

The result of the query, areas $>=30$ and $<=50$, shown in white.

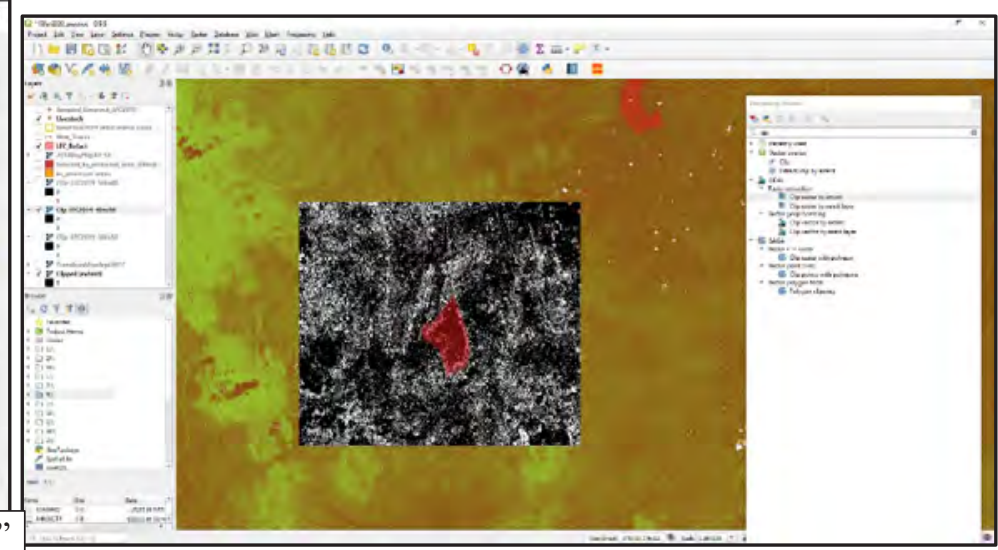

The result of the query, areas $>=40$ and $<=50$, shown in white. 


\section{Creating buffer zones.}

\section{A. Buffer zone of Kenya protected areas.}

Example of a buffer using Kenya protected areas 1998. Download from internet

https://www.wri.org/resources/data-sets/kenya-gis-data; scroll down to Base data and download ke protected-areas.zip; open the *.shp file in QGIS, then click the select feature (shown by red arrow); the selected areas are highlighted in yellow in screen capture 1 . Next, Menu $>$ Vector $>$ Geoprocessing tools $>$ Buffer. OR

Menu $>$ Processing $>$ Toolbox $>$ Processing Toolbox $>$ Vector geometry $>$ Buffer.

Use the parameters in the Buffer dialog box; only a temporary file is created, which can be saved.

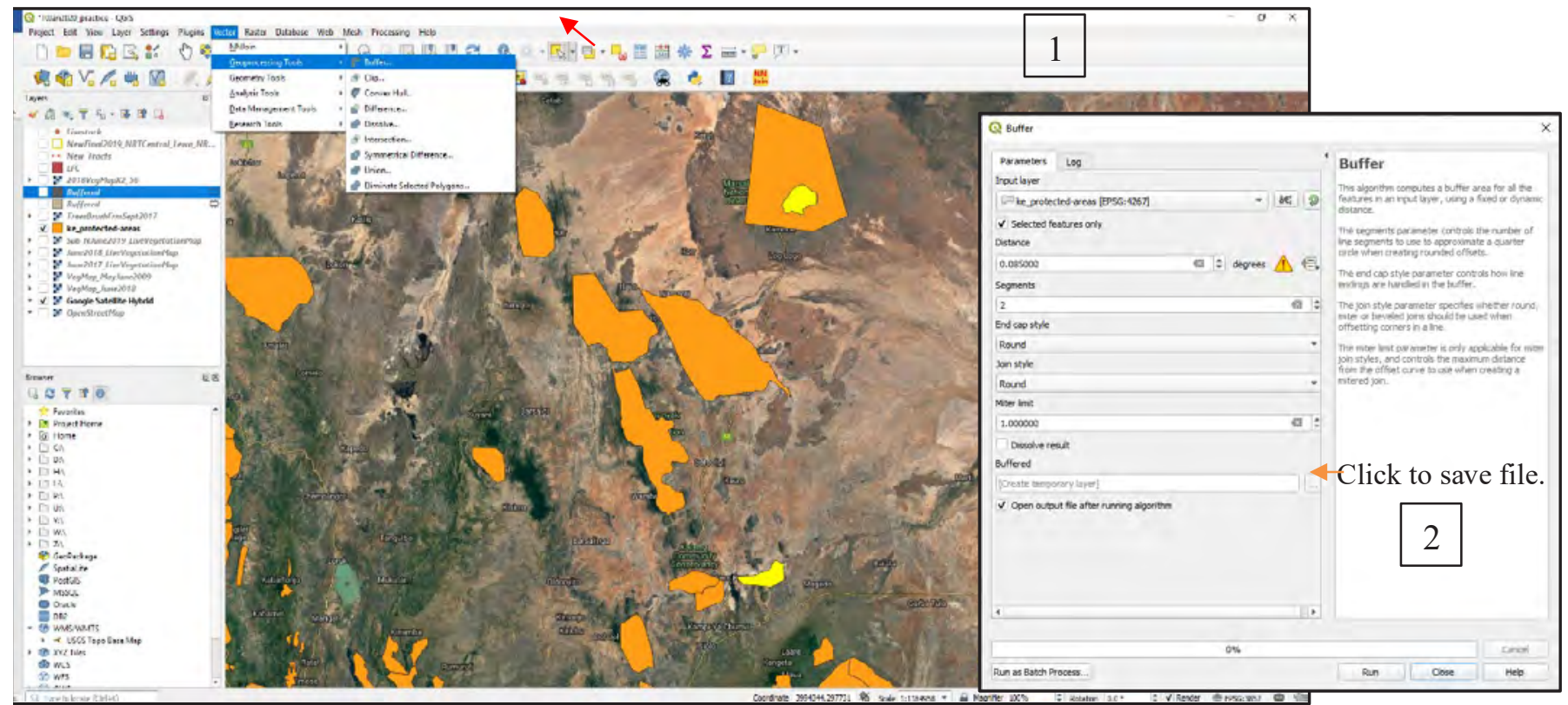

The buffered areas are shown in rust color in screen capture 3 below. The file was opened in QGIS, as specified in the buffer dialog box 2 above.

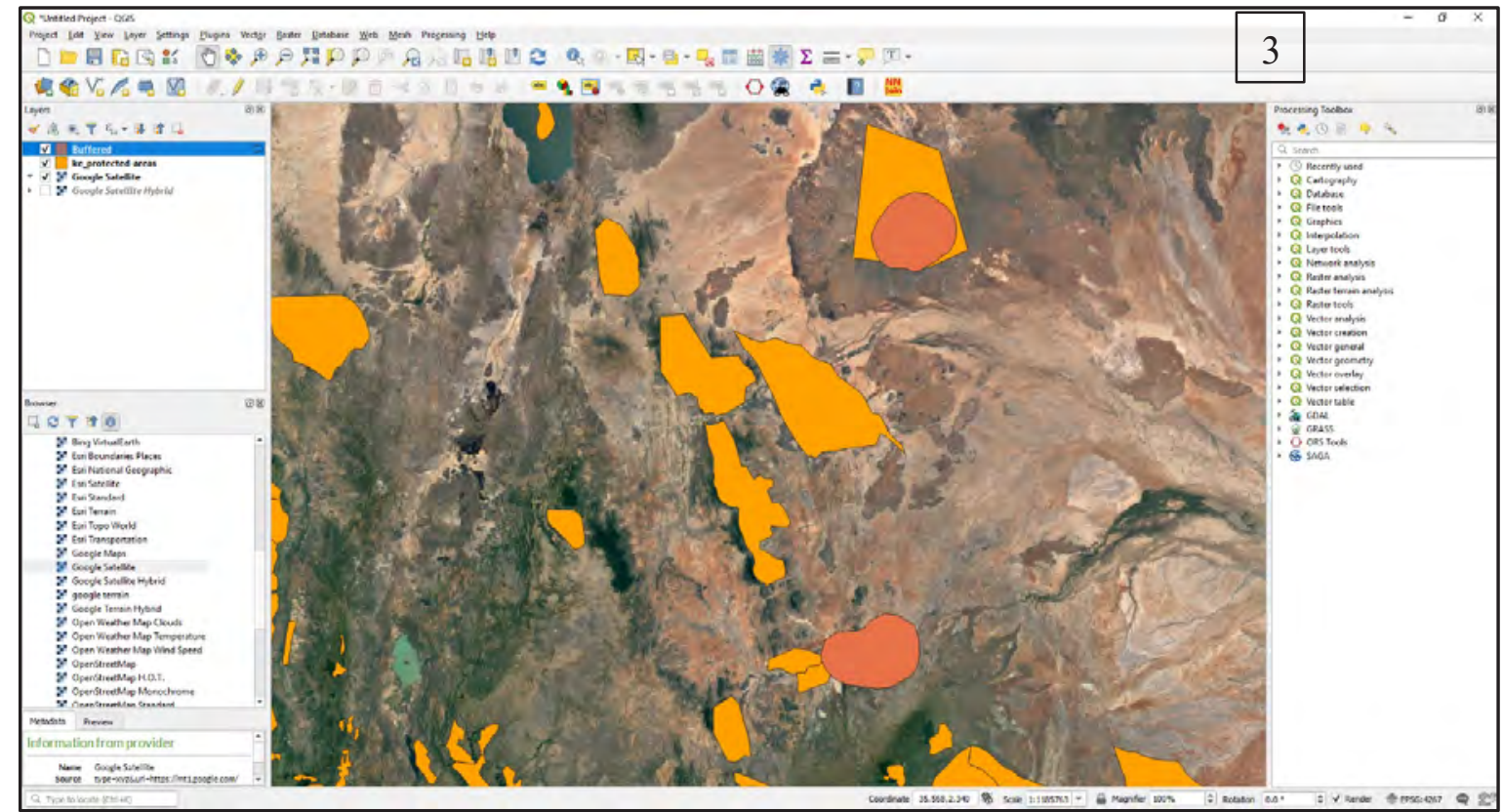




\section{B. Buffer created on point shape file.}

Following is another example of a buffer created with selected features on the Livestock *.shp file.

Follow the parameters shown in the buffer dialog box. Save the file (shown by red arrow).

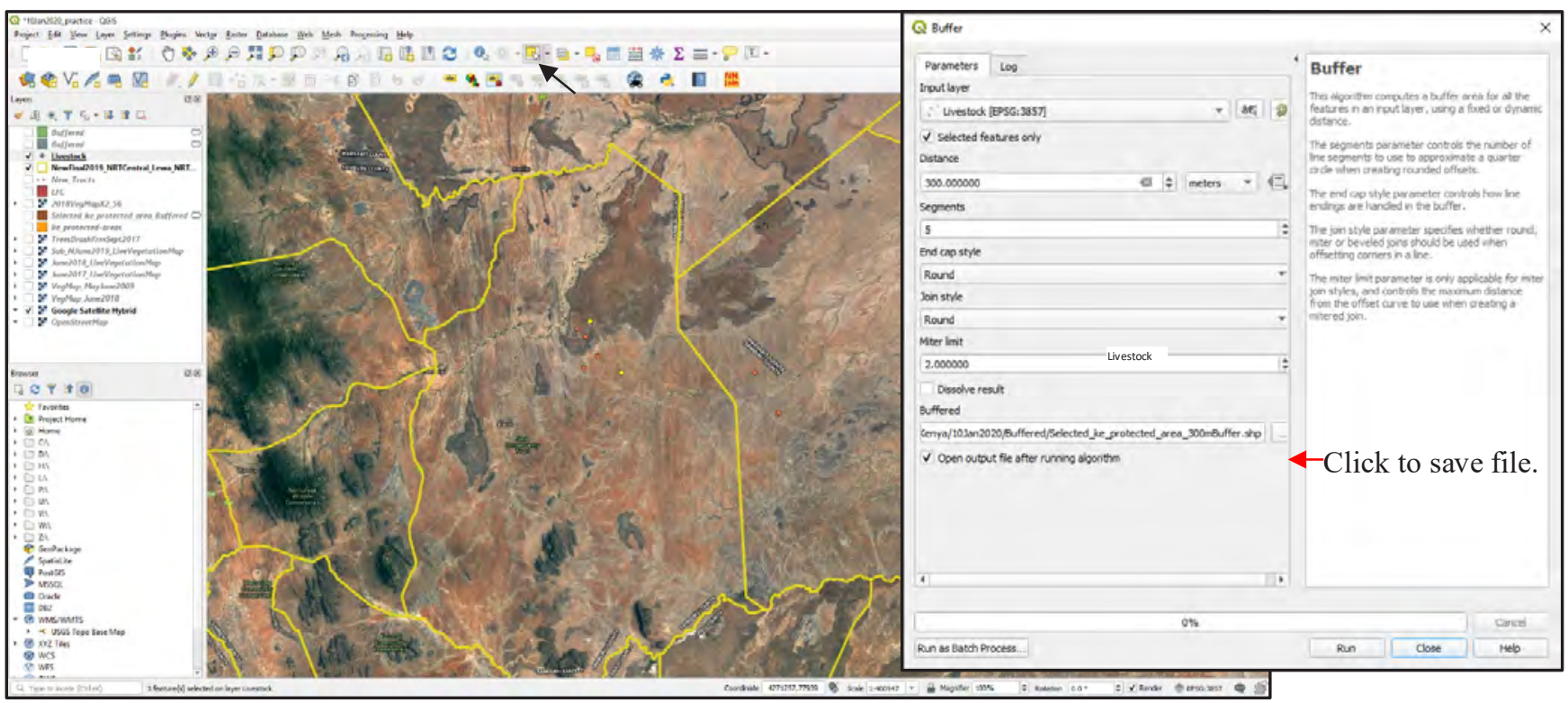

The buffered areas are shown in cyan in the screen capture below. The file was opened in QGIS as specified in the buffer dialog box above.

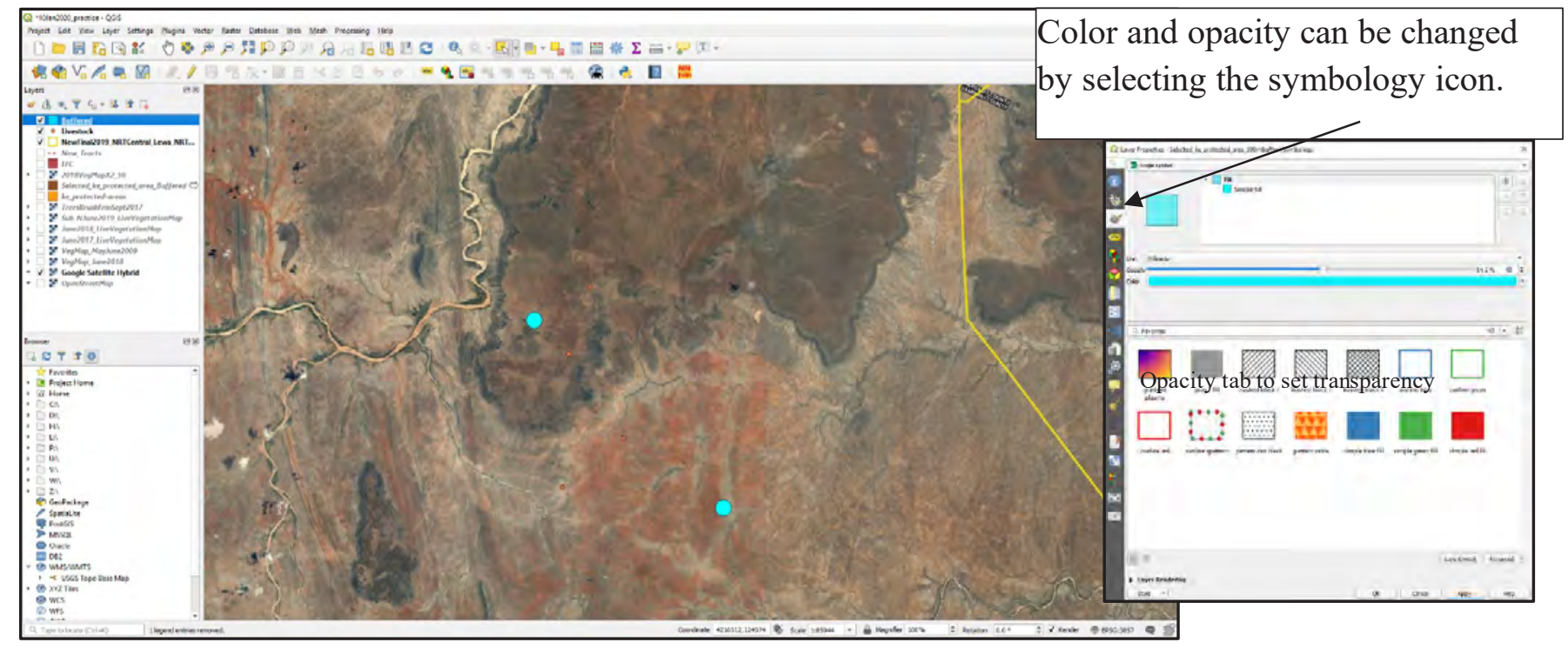




\section{Data export and presentation.}

\section{A. Export shape file data to Excel.}

Example Livestock: Right click the layer, Open attribute table. Click the upper left corner of the identifier (id) column (arrow 1). All fields will get selected; click the copy icon (arrow 2).

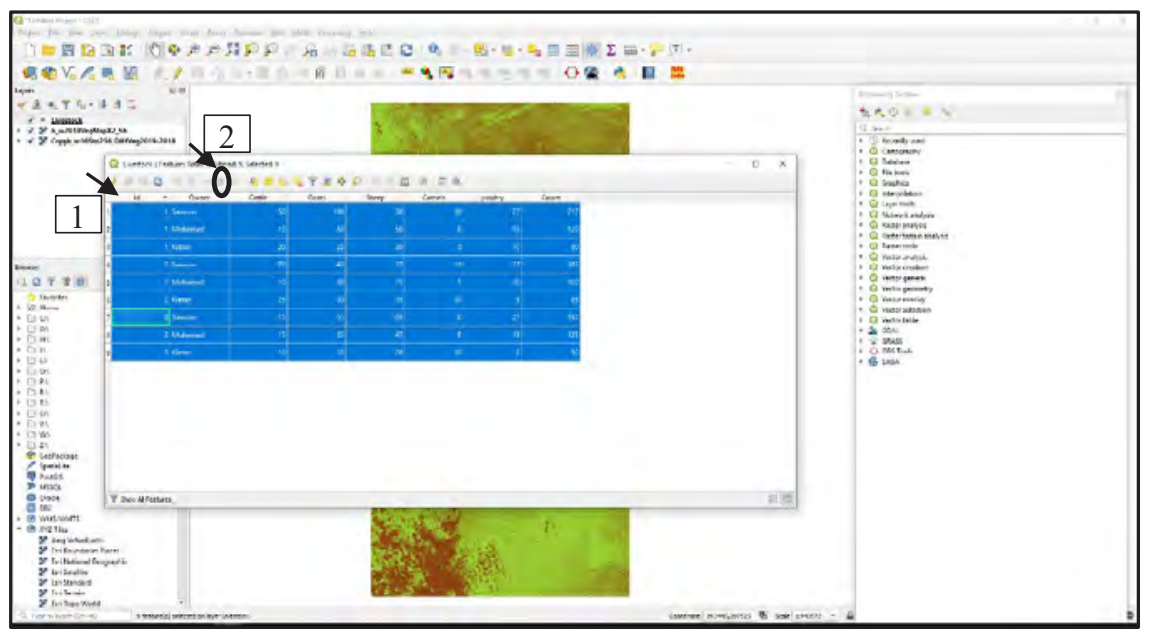

Open a blank Excel sheet and paste copied cells; adjust row height then save in Excel format.

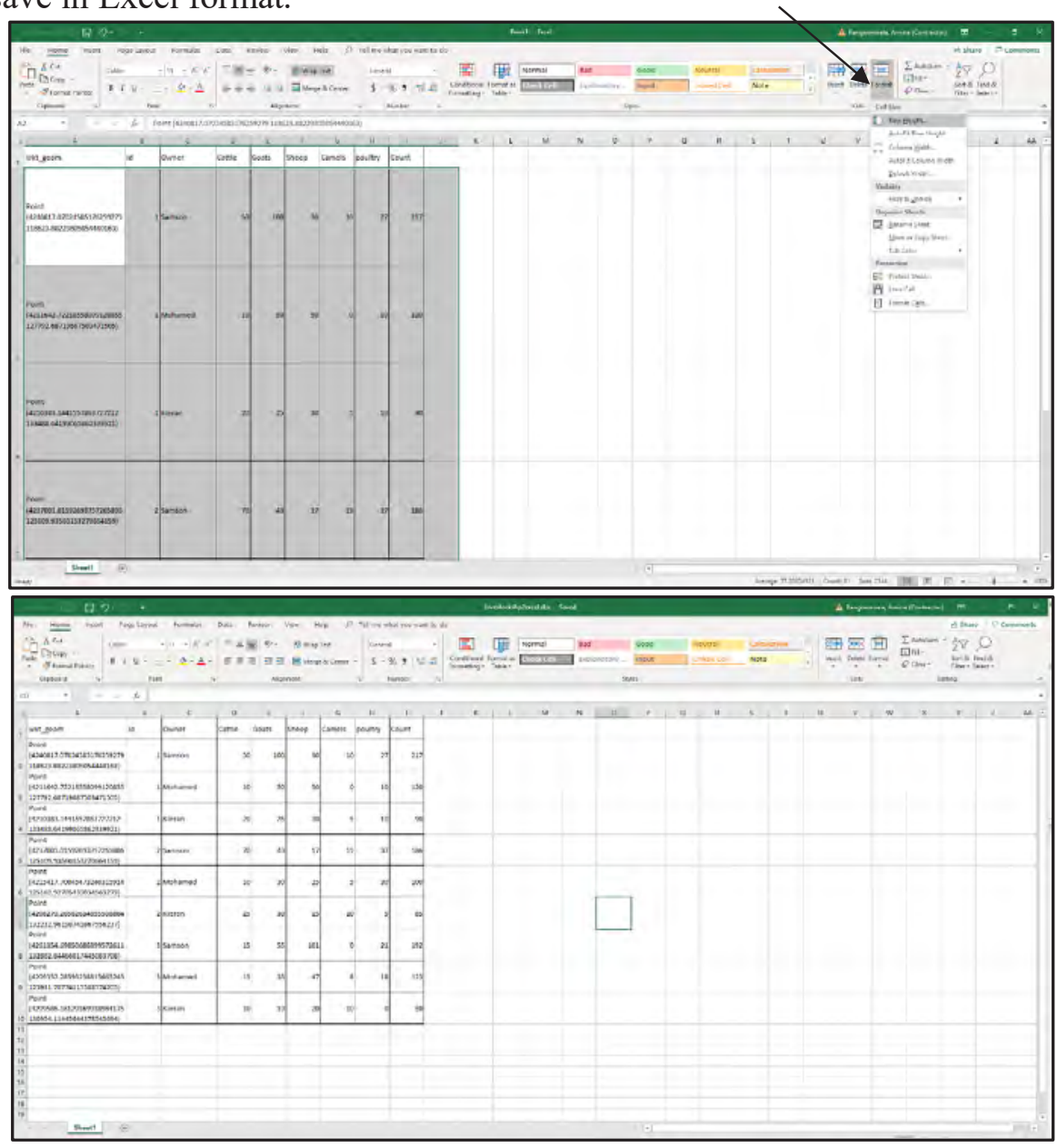




\section{B. Data export to view in Google Earth Pro.}

Exporting the *shp file data to Keyhole Markup Language *.KML (screen capture 1). Example is Livestock shape file. Right click the layer; select Export> Save selected features as. In the dialog box Save Vector Layer select Format as KML; navigate to the folder and select file name; select the parameters shown in screen capture 2 then click OK.

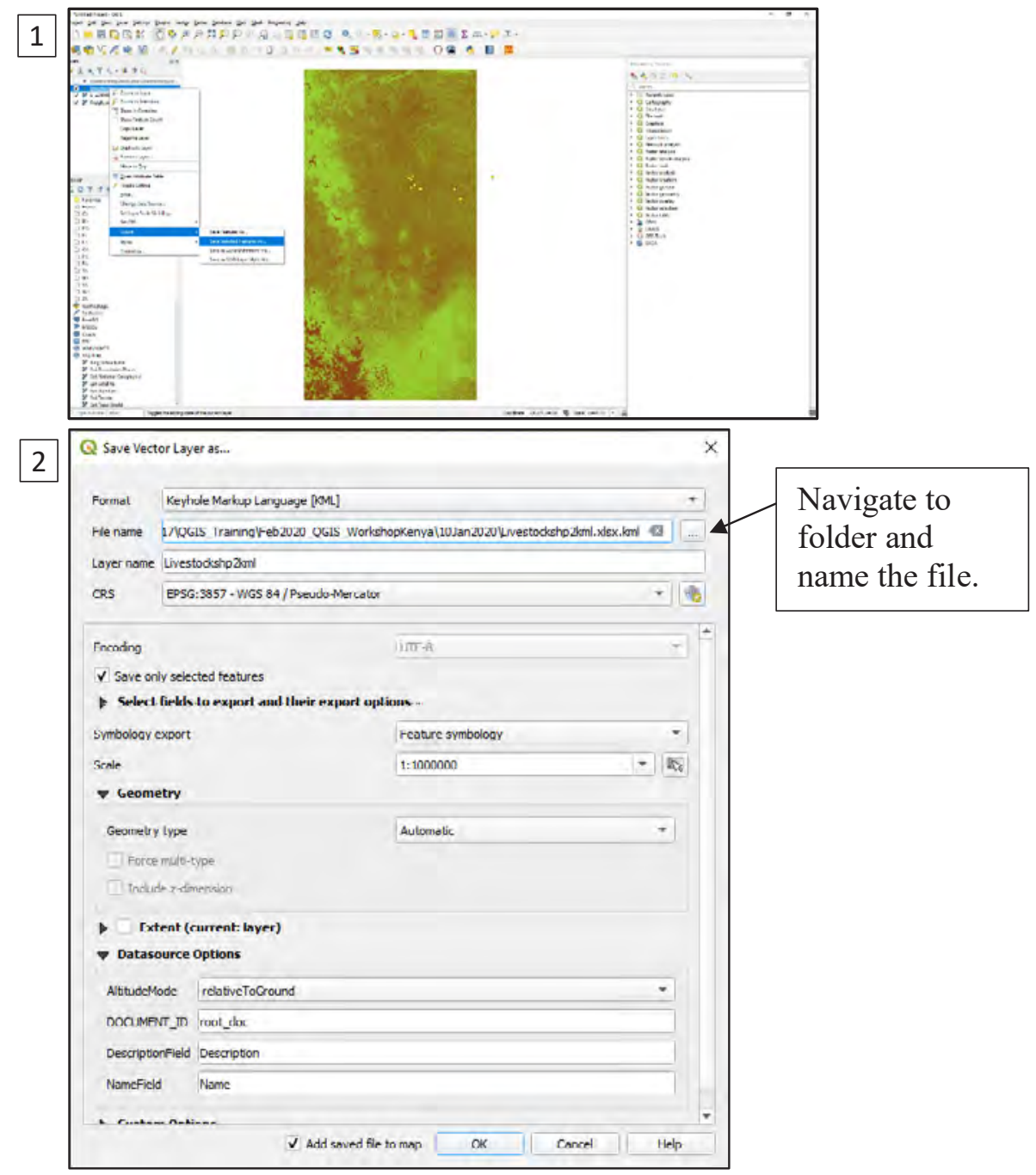

As shown in screen capture 3, Open Google Earth Pro; drag and drop the *.KML file that was created above.

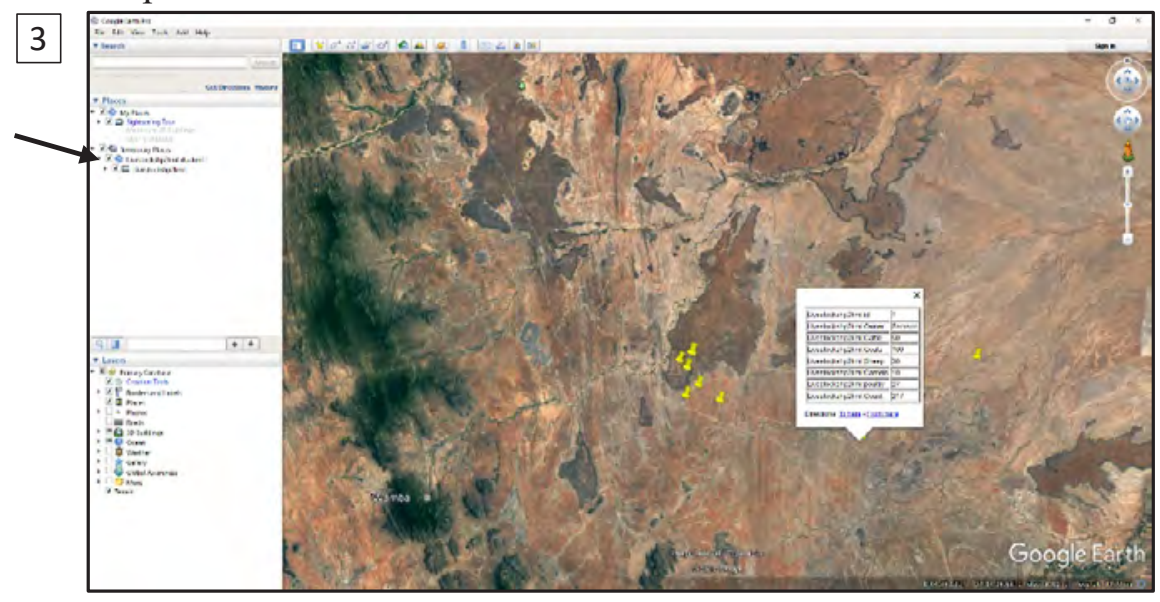




\section{C. Data presentation.}

To make a map layout, select Menu>Project>new print layout; type name of the layout then click OK.
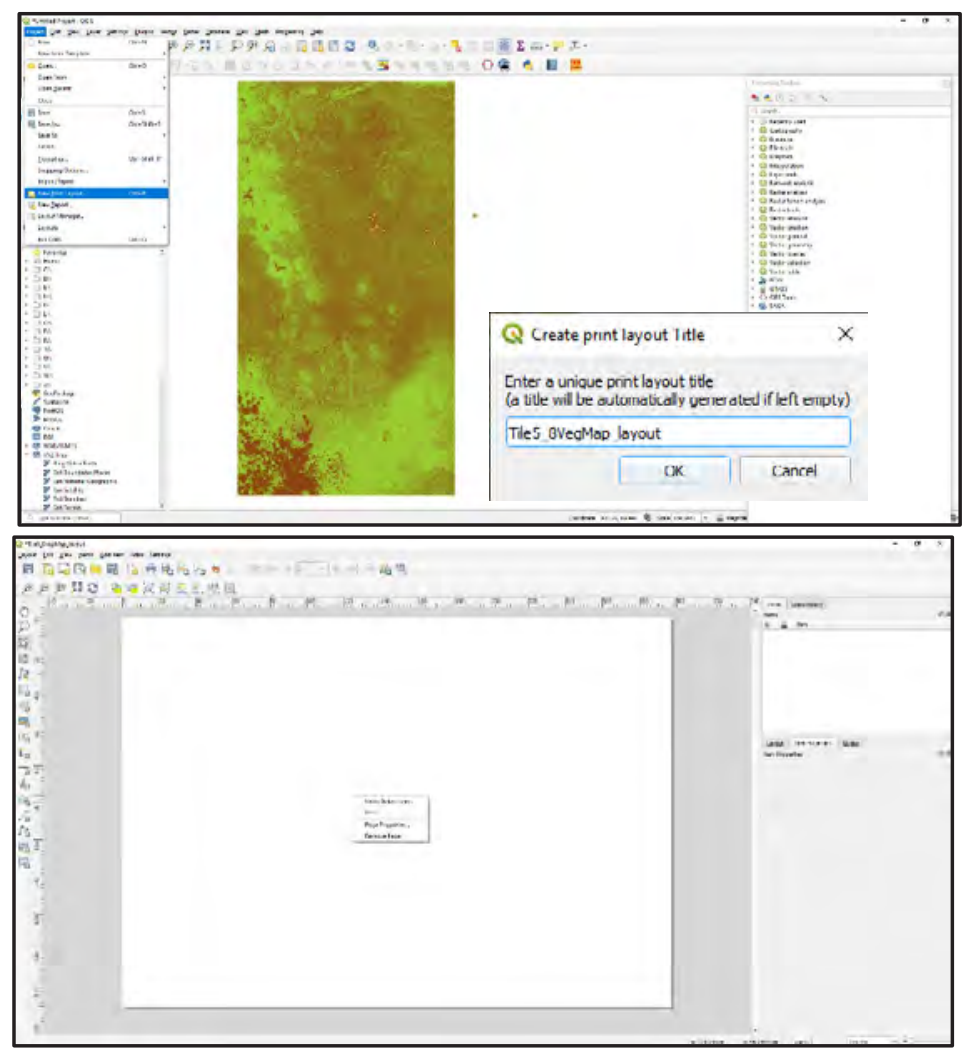

Right click on the map layout to get page.

Select what you want to display on the layout in the QGIS project window. Use icon 1 to set the map window; icon 2 to resize this area (expand at the arrow) and to move the map window, and icon 3 to zoom in and out the map data.

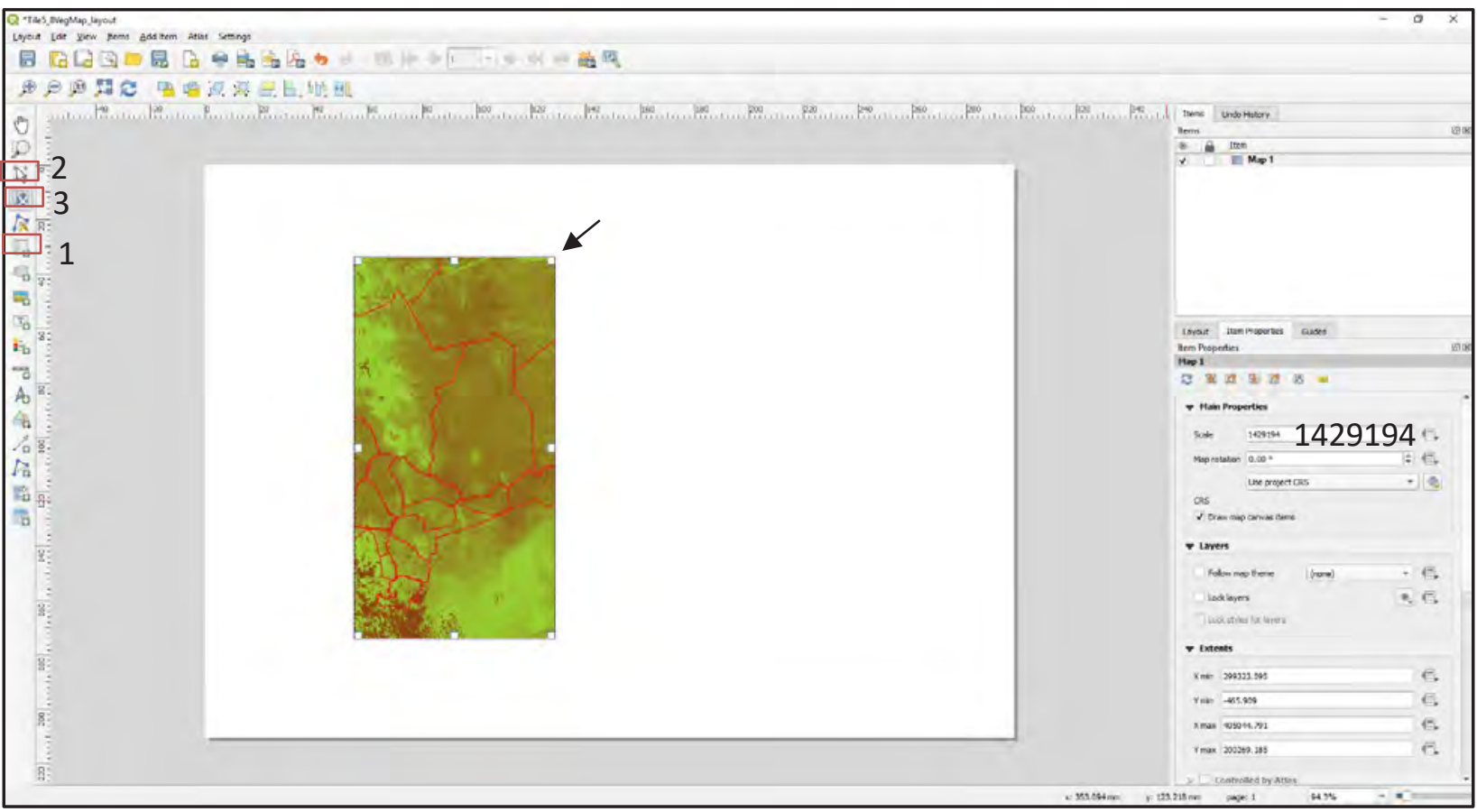




\section{D. Add legend, scale, and north arrow.}

To add a legend, click the icon shown by the red rectangle.

To rename the legend entries, click on the name type in dialog box.

To remove an entry, unclick the radio button Auto update. Highlight the entry to delete and click the minus sign symbol.

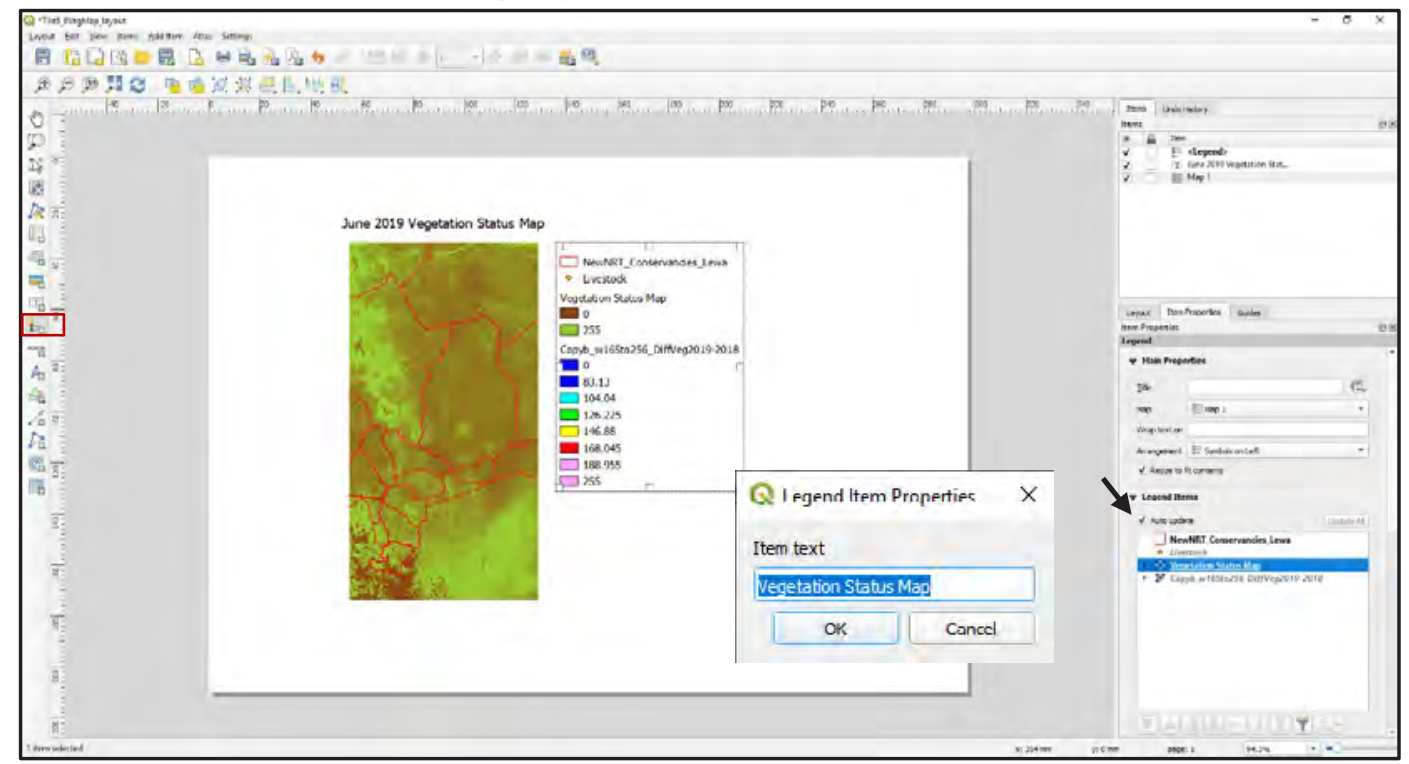

To insert Scale on the map layout, click the scale bar icon shown by the red rectangle and position the scale on the map layout.

To change the style, select desired style from the drop down. Both segments and units can be changed.

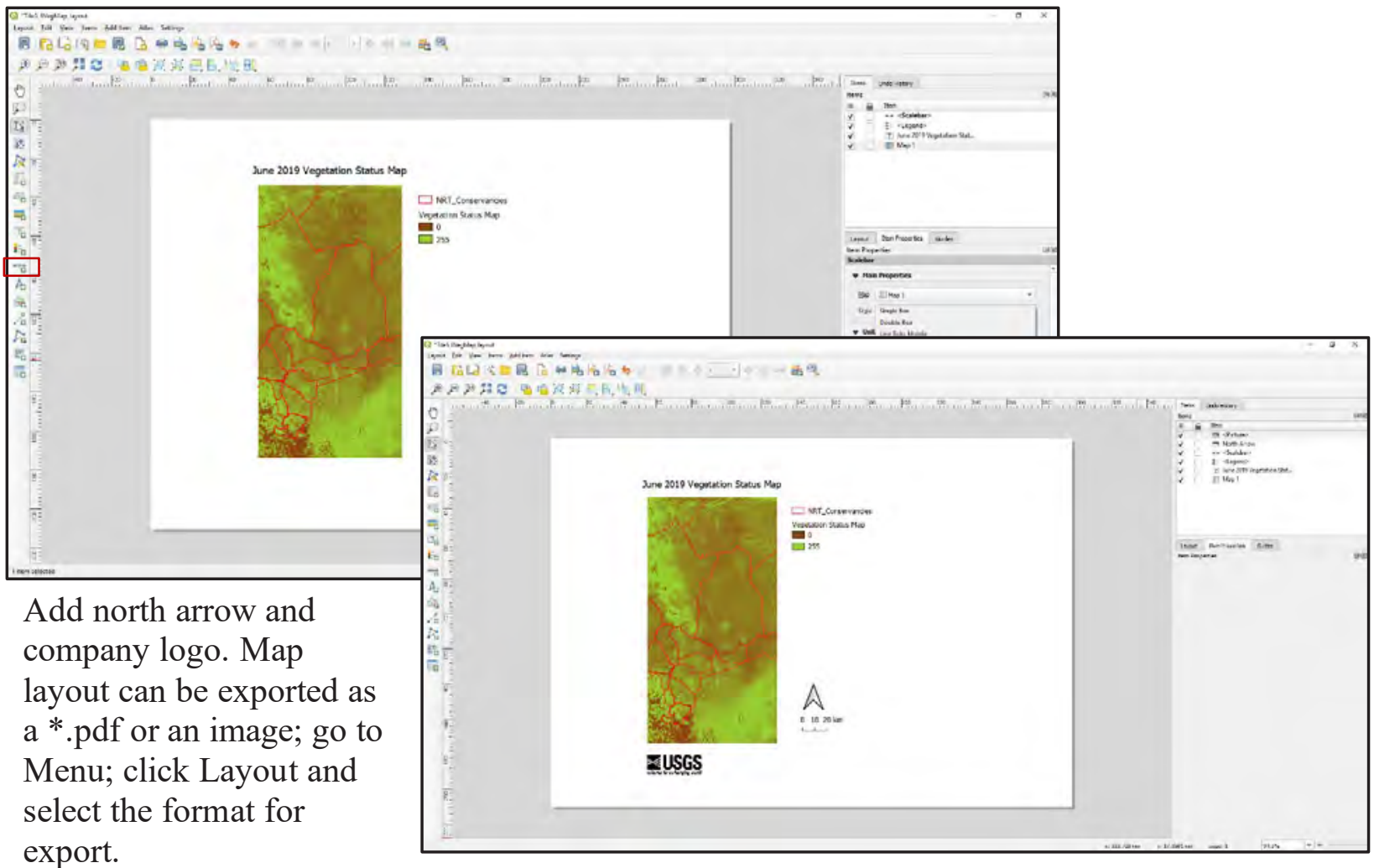




\section{Calculate distances.}

Go to plugins and search openrouteservice (ORS), then click install. This is a very helpful tool but does not currently work with the towns in central Kenya (NRT conservancies) as the open street road map available does not have a detailed road map for these areas.

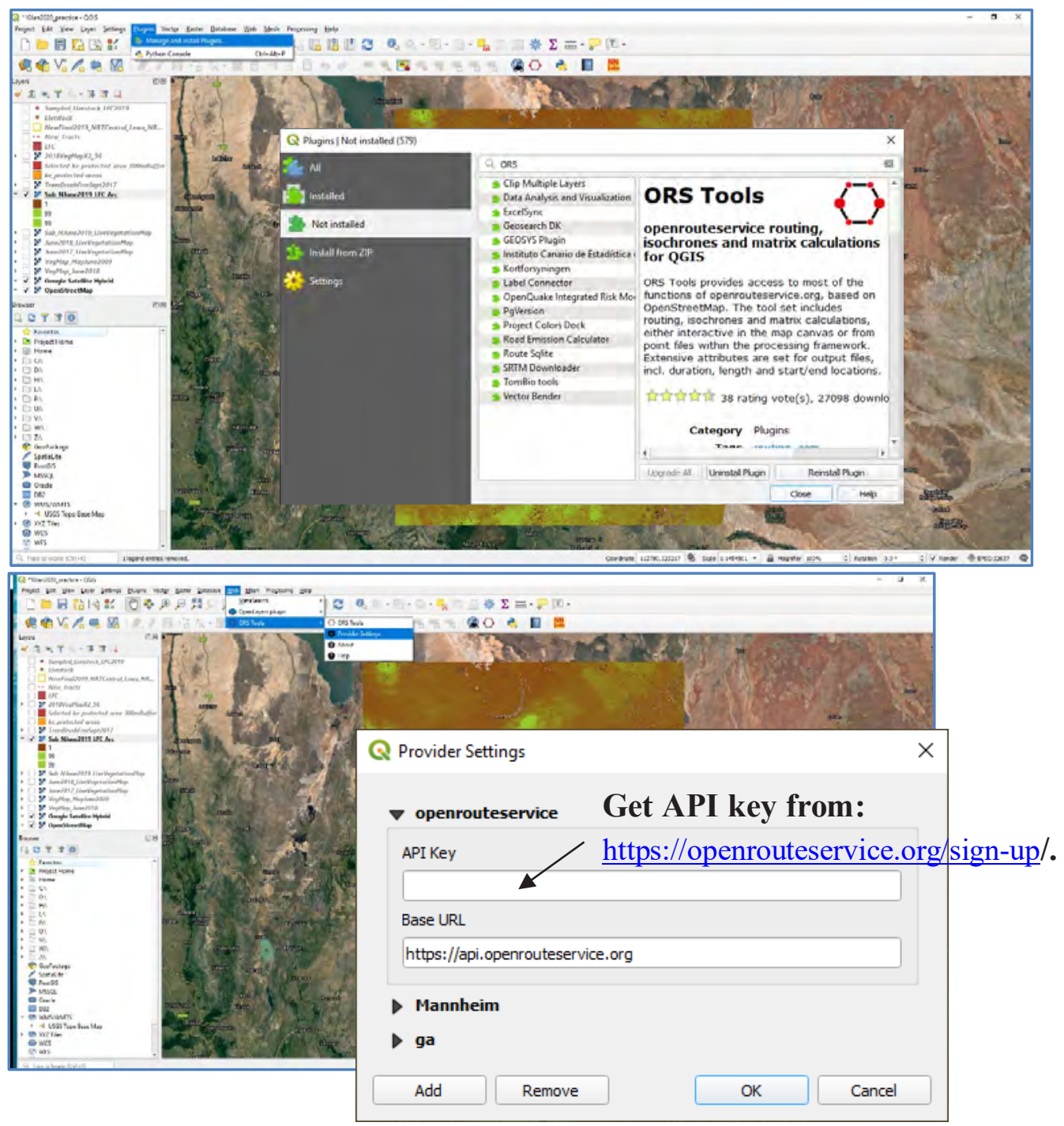



For more information about this publication, contact

Director, Wetland and Aquatic Research Center

U.S. Geological Survey

700 Cajundome Blvd.

Lafayette, Louisiana 70506

For additional information visit

https://www.usgs.gov/centers/wetland-and-aquatic-researchcenter-warc

Publishing support provided by Lafayette Publishing Service Center 
